OAK RIDGE

NATIONAL

LABORATORY

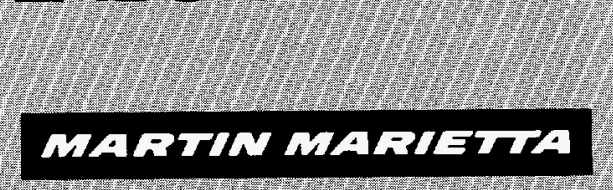

H.

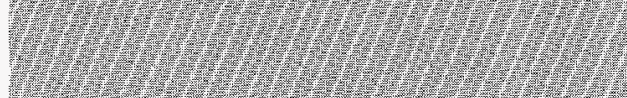

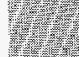

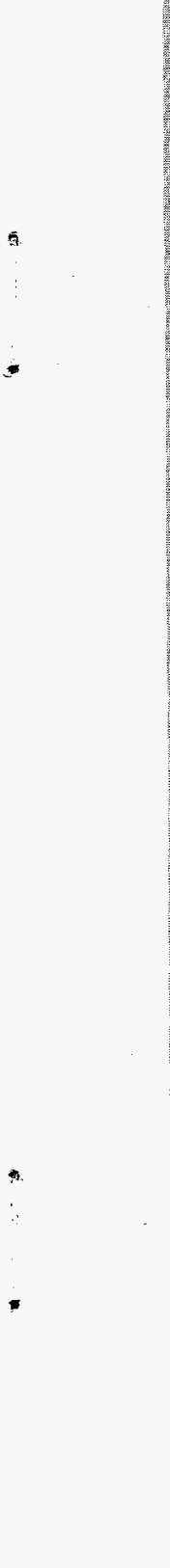

MANAGED BY

MARTIN MABIETTA ENERGY SYSTEMS, INC.

FOR THE UNITED STATES

DEPARTMENT OF ENERGY

\section{Experiences in Effective Use of $\mathrm{Tcl} / \mathrm{Tk}$}

\author{
R. W. Lee
}


This report has been reproduced directly from the best available copy.

Available to DOE and DOE contractors from the Office of Scientific and Technical information, P.O. Box 62, Oak Ridge, TN 37831; prices available from (615) 576-8401, FTS 626-8401.

Available to the public from the National Technical Information Service, U.S. Department of Commerce, 5285 Port Royal Rd., Springfield, VA 22161.

This report was prepared as an account of work sponsored by an agency of the United States Government. Neither the United States Government nor any agency thereof, nor any of their employees, makes any warranty, express or implied, or assumes any legal liability or responsibility for the accuracy, completeness, or usefulness of any information, apparatus, product, or process disclosed, or represents that its use would not infringe privately owned rights. Reference herein to any specific commercial product, process, or service by trade name, trademark, manufacturer, or otherwise, does not necessarily constitute or imply its endorsement, recommendation, or favoring by the United States Government or any agency thereof. The views and opinions of authors expressed herein do not necessarily state or reflect those of the United States Government or any agency thereof. 
ORNL/TM-12811

Computer Science and Mathematics Division

\title{
EXPERIENCES IN EFFECTIVE USE OF TCLTKK
}

\author{
R. W. Lee
}

DATE PUBLISHED -- June 1995

Prepared by the

OAK RIDGE NATIONAL LABORATORY

Oak Ridge, Tennessee 37831

managed by

MARTIN MARIETTA ENERGY SYSTEMS, INC.

for the

U.S. DEPARTMENT OF ENERGY under contract DE-AC05-84OR21400 


\section{DISCLAIMER}

Portions of this document may be illegible in electronic image products. Images are produced from the best available original document. 


\section{CONTENTS}

ACKNOWLEDGEMENTS $\ldots \ldots \ldots \ldots \ldots \ldots \ldots \ldots \ldots \ldots \ldots \ldots \ldots \ldots \ldots \ldots \ldots$

ABSTRACT $\ldots \ldots \ldots \ldots \ldots \ldots \ldots \ldots \ldots \ldots \ldots \ldots \ldots \ldots \ldots \ldots \ldots \ldots \ldots$

1. INTRODUCTION $\ldots \ldots \ldots \ldots \ldots \ldots \ldots \ldots \ldots \ldots \ldots \ldots \ldots \ldots \ldots \ldots \ldots \ldots \ldots \ldots$

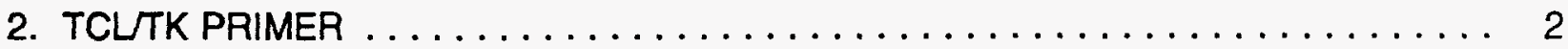

2.1 THE SCRIPTING LANGUAGE $\ldots \ldots \ldots \ldots \ldots \ldots \ldots \ldots \ldots \ldots \ldots \ldots \ldots \ldots$

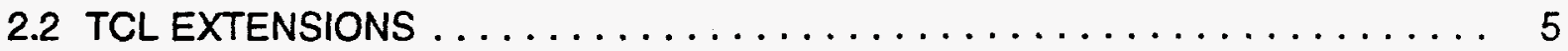

2.3 EXTENDING THE TK WIDGET SET $\ldots \ldots \ldots \ldots \ldots \ldots \ldots \ldots \ldots \ldots \ldots, 6$

2.4 INTERPROCESS COMMUNICATION $\ldots \ldots \ldots \ldots \ldots \ldots \ldots \ldots \ldots \ldots \ldots \ldots \ldots$

2.5 COMPARISON WITH OTHER TOOLS $\ldots \ldots \ldots \ldots \ldots \ldots \ldots \ldots \ldots \ldots 7$

3. PROGRAMMING IN THE SCRIPTING LANGUAGE $\ldots \ldots \ldots \ldots \ldots \ldots \ldots \ldots$

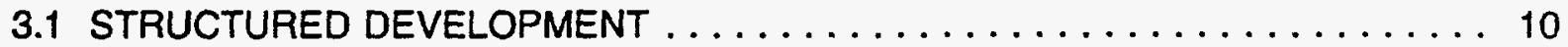

3.2 OBJECT-BASED PACKAGING $\ldots \ldots \ldots \ldots \ldots \ldots \ldots \ldots \ldots \ldots \ldots \ldots \ldots$

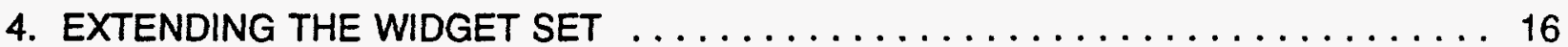

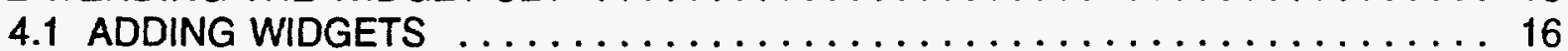

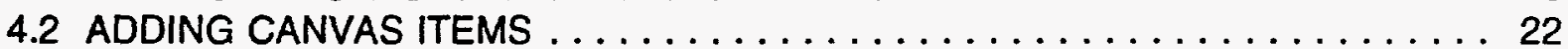

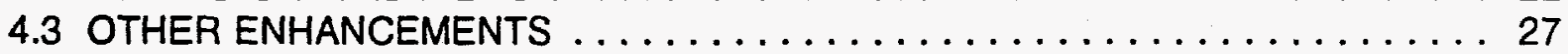

5. EMBEDDING CLASSES AND FUNCTIONS $\ldots \ldots \ldots \ldots \ldots \ldots \ldots \ldots \ldots \ldots \ldots$

5.1 TCL INTERFACES TO C++ CLASSES $\ldots \ldots \ldots \ldots \ldots \ldots \ldots \ldots \ldots \ldots \ldots \ldots \ldots \ldots$

5.2 TECHNIQUES FOR DATA EXCHANGE .................. 36

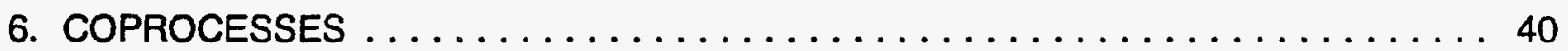

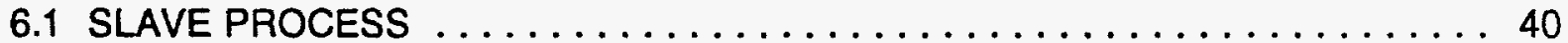

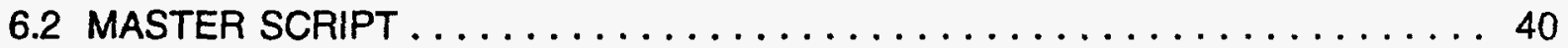

6.3 EMBEDDING WINDOWS $\ldots \ldots \ldots \ldots \ldots \ldots \ldots \ldots \ldots \ldots \ldots \ldots \ldots$

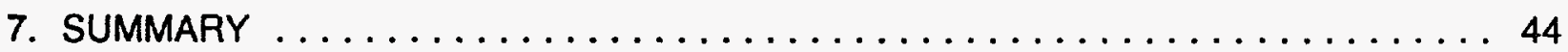

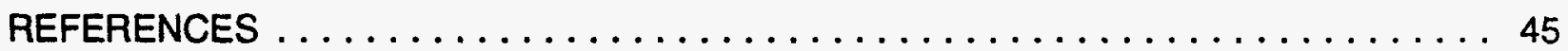




\section{LIST OF FIGURES}

Figure $2-1 \mathrm{Tcl}$ Script to Compute Factorials $\ldots \ldots \ldots \ldots \ldots \ldots \ldots \ldots \ldots \ldots \ldots$

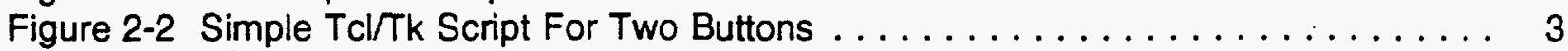

Figure 2-3 Additional Tcl/Tk Commands to Modify Button Appearance .......... 3

Figure 2-4 Example Tcl Functional Extension $\ldots \ldots \ldots \ldots \ldots \ldots \ldots \ldots \ldots . \ldots \ldots$

Figure 2-5 User-Defined Tcl_Appinit() ..................... 5

Figure $2-6$ Canvas Item Creation and Manipulation .................. 6

Figure 3-1 Scripting Language Versus $3 G L$ Decision Metaphor .............. 9

Figure 3-2 Class and Object Attribute Reference .................. 11

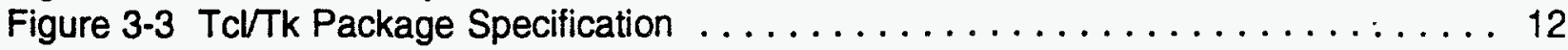

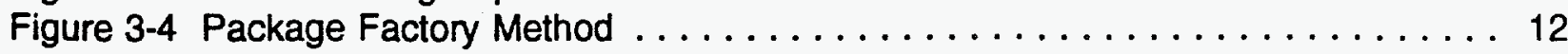

Figure 3-5 Setting the Library Resource to Import Packages $\ldots \ldots \ldots \ldots \ldots \ldots \ldots$

Figure 4-1 Object Attributes for the TkThing Class $\ldots \ldots \ldots \ldots \ldots \ldots \ldots \ldots \ldots \ldots$

Figure 4-2 Widget Configuration $\mathrm{C}_{++}$Code $\ldots \ldots \ldots \ldots \ldots \ldots \ldots \ldots \ldots \ldots \ldots \ldots \ldots$

Figure 4-3 Widget Configuration $\mathrm{C}_{++}$Method $\ldots \ldots \ldots \ldots \ldots \ldots \ldots \ldots \ldots \ldots$

Figure 4-4 C++ Class Method for Handling X Events ................. 19

Figure 4-5 Widget Display Code ............................ 19

Figure 4-6 Widget Object Command Handler . . . . . . . . . . . . . . . 21

Figure 4-7 Widget Factory Command Handler ..................... 21

Figure 4-8 Canvas Item Configuration and Type Definition $\ldots \ldots \ldots \ldots \ldots \ldots \ldots$

Figure 4-9 Canvas Item Create Method ....................... 24

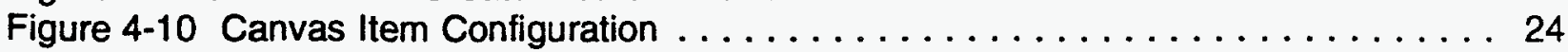

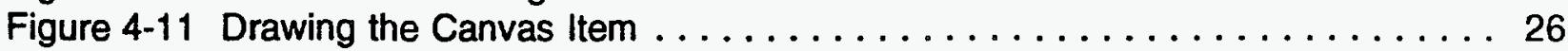

Figure 4-12 Canvas Item PostScript Generation .................. 26

Figure 4-13 Retrieval of Shade Colors From a Tk_3DBorder . . . . . . . . . . 29

Figure 4-14 Code to Render Pixmaps and Bitmaps $\ldots \ldots \ldots \ldots \ldots \ldots \ldots \ldots \ldots$

Figure $5-1 \mathrm{Tcl}$ Derived Class Declaration $\ldots \ldots \ldots \ldots \ldots \ldots \ldots \ldots \ldots \ldots \ldots \ldots \ldots \ldots \ldots$

Figure $5-2 \mathrm{Tcl}$ Class Factory Command Method $\ldots \ldots \ldots \ldots \ldots \ldots \ldots \ldots \ldots \ldots \ldots$

Figure $5-3 \mathrm{Tcl}$ Class Object Command Method .................... 34

Figure 5-4 Tcl Object Subcommand Method ...................... 35

Figure 5-5 Retum of Array Data in Tcl Object Method ................. 37

Figure 5-6 Retuming a Memory Address in a Tcl Object Method ............ 37

Figure 5-7 Tcl Code Snippets . . . . . . . . . . . . . . . . . . . . . . 37

Figure $5-8$ Configuration for Processing a Memory Address $\ldots \ldots \ldots \ldots \ldots \ldots \ldots . \ldots 37$

Figure 6-1 Handling $X$ Events and Standard Input $\ldots \ldots \ldots \ldots \ldots \ldots \ldots \ldots \ldots$

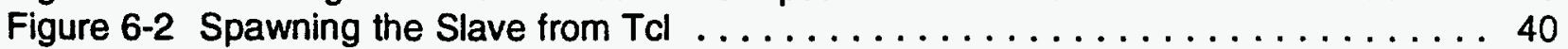

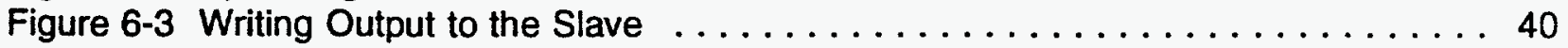

Figure 6-4 Slave Process Checking for Parent Window Handle . . . . . . . . . . . 41

Figure 6-5 Tcl/Tk Commands to Embed Slave Window ................ 41 


\section{ACKNOWLEDGEMENTS}

$\mathrm{TCl}$ and Tk were developed by John Ousterhout of the University of California at Berkeley. Tcl Distributed Programming (TCl-DP) was developed by Lawrence A. Rowe of the University of California at Berkeley and Brian C. Smith of Comell University. 


\section{ABSTRACT}

Tcl/Tk (Tool Command Language and Tool Kit, pronounced "tickle tee-kay") is a scripting language supporting Motif ${ }^{\mathrm{TM}}$ style $\mathrm{X}$ Window interfaces. It is extendible, allowing developers to embed additional functionality as commands in the language. However, the power and flexibility of the system leads to many variations or possibilities in its usage. We describe effective methods for taking advantage of TclTKk to increase productivity and enhance the flexibility and adaptability of applications: writing simple Tcl/Tk scripts, extending the $T c / T k$ widget set, wrapping $\mathrm{TCl}$ commands around existing classes and functions, and building $\mathrm{Tcl} / \mathrm{TK}$ and $3 G L$ coprocesses. Examples are presented from working applications. 


\section{INTRODUCTION}

Imperative in application development today is a graphical user interface (GUI). For the most part, the UNIX world has standardized on the network-based X Window System. Sun's $X$ View (Open Look) and OSF/Motif ${ }^{\text {TM }}$ have battled to be the standard toolkit and widget set, with Motif ${ }^{\text {TM }}$ emerging as the apparent victor. Regardless of the toolkit used (or even if programmed at the $X$ Window level using $X$ lib), much code is necessary to construct windows/widgets, set resource values, establish desired behavior, and respond to input events in an application. Many "builder" tools are available to support interactive construction of interfaces, reducing the time and effort required for some development. However, event handlers and callback routines must be embedded in the generated code. Further, such tools do little to decrease the time spent in the edit-compile-link cycle necessary for each source code modification. Run-time resource managers, such as the Motif ${ }^{T M}$ UIL (user interface language) offer the advantage of separation of form and function and dynamic binding of interfaces, but they too require compilation.

Often, the bottleneck in application development time is the edit-compile-link iteration necessary for even the most cosmetic of changes. This cost is particularly high in a rapid prototype environment [4]. Other factors adversely affecting development of GUI interfaces include the complexity of the toolkit libraries and disproportionately large amount of code dedicated to interface construction as opposed to behavior. For example, even a simple dialog window requires code to construct push button, radio button, text field, option menu, and other widgets. Then, callback routines must be written to respond to the push buttons at least and possibly other widgets as well.

In an effort to reduce the code dedicated to GUI, scripting tools have emerged. HyperCard ${ }^{\text {TM }}$ has been available on the Macintosh for some time now. More recently, Microsoft Visual Basic has emerged as a similar tool for the DOS/Windows environment. In the $X$ Window UNIX community, TCITTk has become the most popular and most used GUI scripting environment, largely due to its extendibility. These scripting tools represent a new paradigm for development of GUI applications, for they attempt to separate an application's form from its function by isolating the construction of interfaces from $3 G L$ source. The interface is maintained in an easy-to-modify, interpreted script.

To the application developer, Tcl/Tk is just one of many alternatives for constructing interfaces. In this report, experience developing working prototype systems with Tcl/TK is described. Four aspects of TclTk development are explored:

- programming in the $\mathrm{TCl}$ scripting language,

- extending the Tk widget set,

- constructing $T C l$ interfaces to $3 \mathrm{GL}$ classes and functions, and

- building TCl and $3 G L$ coprocesses.

Hopefully, this information will benefit someone considering Tcl/Tk as a potential development tool. The reader's familiarity with $\mathrm{Tcl} / \mathrm{Tk}$ and $\mathrm{C}++$ is assumed. 


\section{TCLTK PRIMER}

The best introduction to Tcl/Tk is found in Ousterhout's book [7]. The following excerpt is taken from page 1.

Together they [TCl and TK] provide a programming system for developing and using graphical user interface (GUI) applications. TCl stands for "tool command language" and is pronounced "tickle"; it is a simple scripting language for controlling and extending applications. It provides generic programming facilities that are useful for a variety of applications, such as variables and loops and procedures. Furthermore, $\mathrm{Tcl}$ is embeddable; its interpreter is implemented as a library of $C$ procedures that can easily be incorporated into applications, and each application can extend the core TCl features with additional commands specific to that application.

One of the most useful extensions to $T c l$ is $T k$. It is a toolkit for the $X$ Window System, and its name is pronounced "tee-kay". Tk extends the core TCl facilities with additional commands for building user interfaces, so that you can construct Motif user interfaces by writing $T c l$ scripts instead of $C$ code. Like $T C l, T k$ is implemented as a library of $C$ procedures so it too can be used in many different applications. Individual applications can also extend the base TK features with new user-interface widgets and geometry managers written in $C$.

Tk widgets provide Motif ${ }^{T M}$ behavior but are built solely upon Xlib, thereby allowing $T c l / T k$ to be ported to any UNIX-based $X$ Window environment. That is, TK widgets are not Motif' widgets.

Tcl/Tk is exhaustively described in Ousterhout's book, available in PostScript in four parts from the anonymous FTP site sprite. berkeley. edu. Here we provide a very brief and general description of Tcl/TK and introduce the concepts discussed in the remainder of the paper.

\subsection{THE SCRIPTING LANGUAGE}

Essentially, TCl and Tk are libraries for building shell interpreters. Tcl statements are read and processed by an interpreter program. The distributions provide the means for building default shells. Unextended, the Tcl shell is tclsh, which supports the basic language constructs (e.g., variable manipulation, iteration, control) but no GUI capabilities. The unextended Tcl/Tk shell is wish (windowing interactive shell), which supports many commands for creating widget objects and controlling windows. A great many extensions to Tcl/Tk have been developed and made available via the internet. These extensions provide the components (e.g., source code, object modules, and/or libraries) necessary to build new shell interpreters with the ability to process new commands and features. Additional sources

1 The tclMotif package available from harbor.ecn.purdue.edu provides an interface to Motif widgets. 
of information are the harbor. ecn.purdue. edu FTP site and the comp. lang.tcl news group.

The underlying paradigm for the Tcl shell interpreter is the UNIX command line. In fact, each statement in the language is a command line with options and arguments specified in the familiar UNIX shell format (i.e., options identified with a preceding dash). It is important for the developer to understand that even control statements such as if-elseif-else are single-line commands. Commands may be combined in lists to form procedures, which are defined with optional arguments and default values.

All variable values are stored as strings, making arithmetic computation slow, since conversions to and from numeric formats are necessary. Variable values are stored in a hash table indexed by variable name and can be scalar, lists, or arrays. Lists, stored as strings with delimiters between values, are the vehicle for most of the data manipulation as well as code interpretation in the language. Arrays are associative, implemented as individual hash tables with indexes always treated as strings. These mechanisms can be used in very powerful and effective ways from the viewpoint of the developer. However, one must always be mindful of the potential performance problems inherent in processing null-terminated $\mathrm{C}$ strings.

In and of itself, tclsh is as effective a scripting language as any of the UNIX shells, and in some ways awk or perl. Figure $2-1$ shows an example script for computing factorials for a set of numbers and illustrates the compactness of the language.

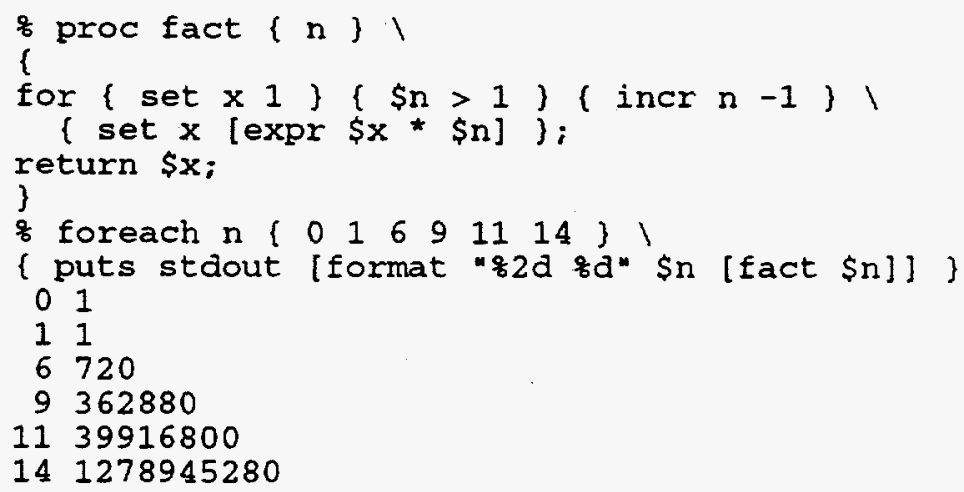

Figure 2-1 Tcl Script to Compute Factorials

Tk's utility becomes immediately clear in the simple example of Figure 2-2, which produces a window with two buttons. As in Motif ${ }^{T M}$, a left mouse button release activates TK buttons. In the example, activation of the buttons results in new xterm windows with green and blue backgrounds, respectively. Figure 2-3 shows further commands to change the appearance of the buttons. 
\& wm withdraw.

\% button . one -text "Green" I -command \{ exec xterm -bg Darkgreen -fg white \& \}

\& button . two - text "Blue" । -command \{ exec xterm-bg MidnightBlue-fg white \&

\& pack .one .two -side left

\& wm deiconify.

\section{Figure 2-2 Simple Tcl/Tk Script For Two Buttons}

o . one configure 1

-background DarkGreen -activebackground gray 75 ।

-foreground yellow -activeforeground \#005f00

-font $9 \times 15 b o l d$

. two configure -background MidnightBlue -activebackground gray 75

Figure 2-3 Additional Tcl/Tk Commands to Modify Button Appearance

int

TclMath: : ProcessRandomcommand (

ClientData data,

Tcl_Interp * $\cdot$ interp.

int arge,

char * argv[

1

char buffer[ 64 ];

if ( ! _tclRanaInitflag)

1

srand48 ( time( NULL) );

$\bar{\xi}$ tclRandInitflag $=1$

sprinte( buffer, "qlg*, arand48());

TCl_SetResult ( interp, buffer, TCL_VOLATILE);

return TCL OK:

) // TclMath: : ProcessRandomcommand

..

void

TClMath: :Registercommands( Tcl_Interp - interp)

!

TCl_createcommand (

interp.

"random*

(TCl_CmaProc *) ProcessRandomcommand,

NULL,

NULL

):

j // TclMath: : RegisterCommands

Figure 2-4 Example Tcl Functional Extension 


\subsection{TCL EXTENSIONS}

The real power of $T c l$ is its extendibility. Developers extend it by compiling and linking a new shell interpreter with procedures "registered" to be called for new commands. Tk is such an extension. There are two modes for adding new functionality: functional, and objectbased. In the functional mode, new commands correspond to individual functions. Refer to Figure 2-4 for code snippets illustrating a simple $\mathrm{C}++$ implementation of a "random" command. Note that the methods in the example must be declared static in the class declaration.

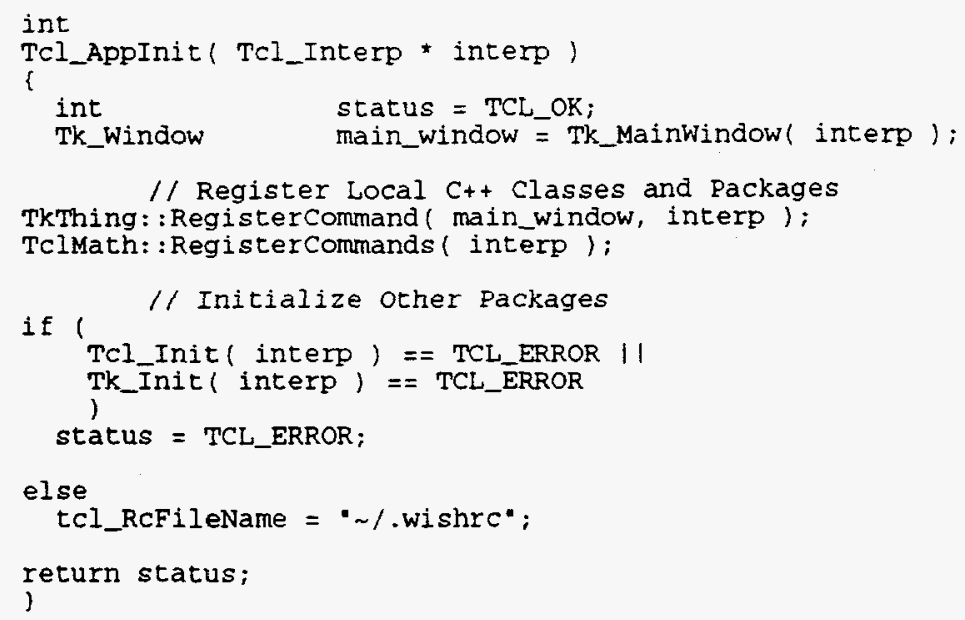

Figure 2-5 User-Defined TCl_AppInit()

Developers hook into TClTK and extension libraries by implementing a function named TCl_AppInit () in which necessary initialization (e.g., registering commands) is performed. A template version is provided with the Tcl distribution. The main () routine is linked from the $\mathrm{Tcl}$ or Tk library. Figure 2-5 illustrates a user-defined TC1_AppInit (). Note the standard initialization of the Tcl and Tk libraries via TCI_Init () and Tk_Init (), respectively. Were we to produce an extension available to the internet, we would generate such a function.

Object-based extensions require at least two functions: one to implement a "factory" or object creation command, and another to implement the individual object command. ${ }^{2}$ This is the paradigm of the Tk widgets. In Figure 2-2, the objects named . one and . two are created with the factory butt on command. Figure 2-3 shows use of the commands . one and . two. Within the factory command implementation, the command for the newly created object must be registered (via TC1_CreateCommand ()). Similarly, when the object is deleted, its command must be unregistered (via TCl_DeleteCommand ()).

2 The concept of factory and instance methods is described by Cox [3]. 


\subsection{EXTENDING THE TK WIDGET SET}

As stated earlier, Tk extends $\mathrm{Tcl}$ with a collection of widgets and window management functions. Developers may add widgets using the object-based paradigm and meeting the requirements for widgets. A widget implementation must include procedures to:

- configure the widget,

- handle X Window events,

- display the widget,

- destroy the widget,

- respond to widget commands,

- create widgets.

The last two are the object and factory commands, respectively. Configuration involves setting and retrieving attribute values. Tk supplies constructs for automatic parsing as well as storage and retrieval of attributes as fields in a data structure. Example uses of these constructs are presented in Section 4.

One of the most powerful of the Tk widgets is the canvas. Within canvases, one creates and manipulates graphical canvas items of various types. Figure 2-6 shows a Tcl/Tk script which creates a rectangle within a canvas and defines an event handler for mouse motion with button one pressed. The requirements for additional canvas item types are more

\& canvas .can -width 200 -height 200 ;

\& pack . can -side top:

$\&$. can create rectangle $50 \quad 50 \quad 150 \quad 150 \quad$ । -tags myrect ।

- Eill red ।

-outline white 1

-width 2

\% . can bind myrect $<B 1-$ Motion $>1$

$\{$. can coords myrect $8 x$ of [expr $8 x+100]$ [expr $8 y+100]$ \}

Figure 2-6 Canvas Item Creation and Manipulation

stringent. Functions for the item type must be provided to:

- create an item instance,

- configure,

- store and retrieve coordinates,

- delete,

- draw,

- compute the distance to a point,

- determine overlap with a rectangular region,

- generate a PostScript representation,

- scale,

- translate. 
Items processing text must supply text handling functions as well.

\subsection{INTERPROCESS COMMUNICATION}

There are two communication mechanisms available within Tcl/Tk. First, separate Tcl/Tk processes connected to the same $X$ Window server may communicate using $X$ events via the send command. Second, Tcl has built-in facilities for processing files and command pipes, allowing a Tcl/Tk application to operate as a coprocess. Both of these mechanisms allow distributed Tcl/Tk implementations. In addition, the Tcl-DP (distributed processing) extension, also from Berkeley, provides commands for socket communication and registration of procedure handlers. Of course, the developer can always build communication facilities in $\mathrm{C} / \mathrm{C}++$ and implement $\mathrm{Tcl}$ commands to manipulate them.

\subsection{COMPARISON WITH OTHER TOOLS}

$\mathrm{Tcl} / \mathrm{Tk}$ is a unique user interface development tool. Not only is it interpreted, requiring no compilation, it offers powerful extensibility at the command or object level. Builder tools take a different approach. They generally produce source code to build interfaces defined with a very powerful point-and-click layout editor. Resource management systems like the Motif ${ }^{\mathrm{TM}}$ UIL offer a compromise by compiling into an intermediate form which is more or less interpreted at run time. Most builder tools will generate UIL as well.

For comparison, we evaluate the three approaches in terms of features desired in interface development: extensibility, time required to complete a change, and effort required to build

\begin{tabular}{||l|c|c|c|}
\hline & Builder Tools & Motif ${ }^{\text {TW UIL }}$ & TalTk \\
\hline Extensibilty & low & very low & very high \\
\hline $\begin{array}{l}\text { Time to complete } \\
\text { change }\end{array}$ & high & moderate & low \\
\hline $\begin{array}{l}\text { Effort in } \\
\text { constructing initial } \\
\text { interface }\end{array}$ & low & high & very high \\
\hline
\end{tabular}

Table 2-1 Interface Development Comparison

the initial interface. Refer to Table 2-1. 


\section{SUMMARY}

$\mathrm{Tcl} / \mathrm{Tk}$ provides a set of libraries for building shell interpreters. Inherent in $\mathrm{Tcl}$ are language constructs for flow control, iteration, and modularization, and list and array as well as scalar variable storage. Language structure follows the UNIX command line paradigm. Tk provides commands for Motif ${ }^{\text {TM }}$ style widgets implemented using Xlib. Tcl/Tk's real power lies in its extendibility, allowing the developer to add new commands and graphic widgets to the language.

In the following sections, we examine the four modes of development using Tcl/Tk: the scripting language, widget extensions, interfaces to $3 G L$ code, and writing coprocesses. 


\section{PROGRAMMING IN THE SCRIPTING LANGUAGE}

$T c l$ is to GUI interfaces as so-called " $4 G L$ " languages are to database management system (DBMS) interfaces. Thus, Tcl presents many of the same types of problems as do 4GLs:

- provides minimal support for programming structure,

- strains modular cohesion and coupling,

- forces run-time debugging,

- lacks debugging support tools,

- magnifies performance issues due to command interpretation.

Procedures, iteration, control, lists, arrays, and scalar variables are the only $\mathrm{Tcl}$ language constructs. Array variables must be global and cannot be passed as procedure parameters, forcing a dependence upon global variables. Variable declaration is implicit, so name misspelling and other errors are found at run time, often after much investigation. Except for matched braces, procedure syntax is not checked until run time, for the procedure is itself merely a string of commands to be executed. Commands are interpreted, making loops perform poorly.

However, simple operations can be scripted in Tcl for immediate testing, avoiding the compilation and linking of a $\mathrm{C}$ program. Changes are made quickly and tested immediately. Frequently, the developer is faced with an implementation choice:

- use only the scripting language,

- build functionality in $\mathrm{C} / \mathrm{C}++$,

- use a combination of scripting language and $\mathrm{C} / \mathrm{C}++$.

Three factors contribute to this decision:

- complexity,

- performance criticality,

- volatility.

The more complex the code, the more likely the scripting language will become a hindrance in the debugging effort. Similarly, there are situations, such as rapid real-time display, in which performance requires use of $\mathrm{C} / \mathrm{C}++$. On the other hand, highly volatile or changing objects or functions benefit from the flexibility and quick turnaround of the interpreted language. Figure 3-1 pictorially represents the interaction among these factors, assuming complexity and performance coincide.

In a prototyping environment, it is often desirable to use the scripting language until design decisions solidify, upon which critical objects and functions can be implemented in $\mathrm{C} / \mathrm{C}++$. Similarly, but less likely, it may be determined that operations should be moved from $3 G L$ to the interpreted language to make changes easier. 


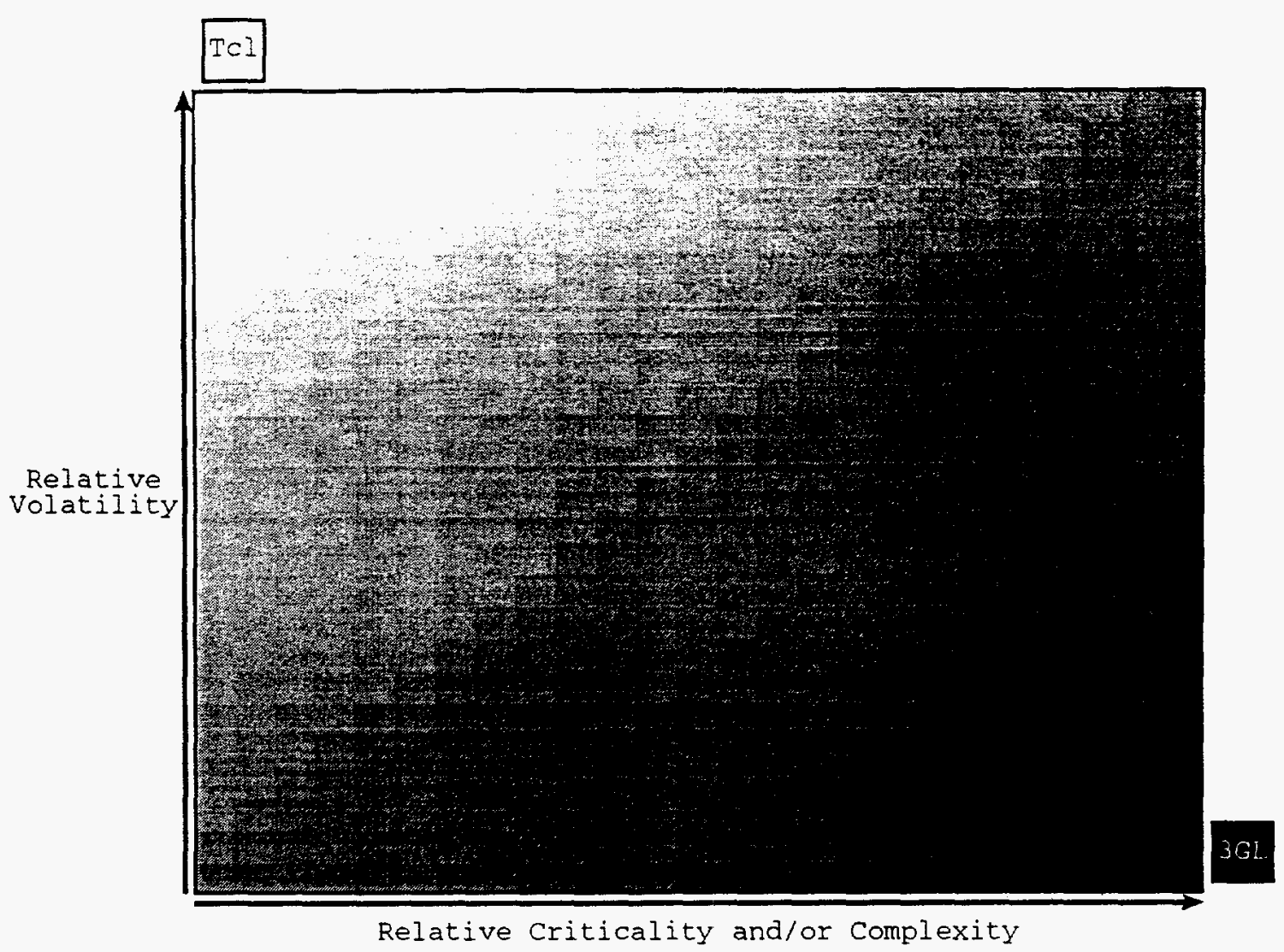

Figure 3-1 Scripting Language Versus 3GL Decision Metaphor

\subsection{STRUCTURED DEVELOPMENT}

In order for the Tcl scripting language to be used effectively, a structured framework for achieving software engineering goals, modifiability, efficiency, reliability, and understandability, must be adhered to. Software engineering concepts which make those goals achievable include: [2]

- abstraction,

- information hiding,

- modularity,

- Iocalization,

- uniformity,

- completeness,

- confirmability.

$\mathrm{Tcl}$ language constructs do little to support these concepts. However, with the exception of the last two, the identified concepts can be realized by building upon the language constructs 


\begin{tabular}{|c|l|}
\hline Principle & \multicolumn{1}{|c|}{ Strategy } \\
\hline abstraction & Implement object-based packages \\
\hline information hiding & $\begin{array}{l}\text { Store attribute-value pairs for package instances in an } \\
\text { array accessed only by the package methods }\end{array}$ \\
\hline modularity & $\begin{array}{l}\text { Implement package methods with procedures whose } \\
\text { first parameter is the name of the object instance. } \\
\text { Other than the package attribute array, all values } \\
\text { manipulated by methods are passed as arguments or } \\
\text { defined locally }\end{array}$ \\
\hline localization & $\begin{array}{l}\text { Implement all method functions within package } \\
\text { framework }\end{array}$ \\
\hline uniformity & $\begin{array}{l}\text { Follow the above guidelines for all package } \\
\text { implementations }\end{array}$ \\
\hline
\end{tabular}

Table 3-1 Tcl Programming Strategies to Achieve Software Engineering Concepts

and developing with discipline. For example, there are no "structures" or "records" to directly support data abstraction, but associative arrays and lists can be used for that purpose. Table 3-1 summarizes strategies to achieve the first five concepts.

\subsection{OBJECT-BASED PACKAGING}

Object-based extensions have been built for Tcl/Tk using only the scripting language. TclDP provides such an environment. We have attempted to construct an object-oriented environment (supporting inheritance) adhering to Tcl-DP structure (refer to Appendix G) but found the performance hits for searching up class hierarchies too prohibitive. The interested reader should examine Tcl-DP as well as the scheme we used.

We've found a "class package" concept, modeled after the Ada package construct, to provide an effective object-based environment that fosters reuse. A package consists of a set of methods for creating and manipulating object instances of the class and a global array variable for storing class and object attributes. Class attribute values are referenced by attribute name, and object attribute values are referenced by object name and attribute name,

class attribute: Barwindow(count)

object attribute: Barwindow(barwino, FileName)

Figure 3-2 Class and Object Attribute Reference 
as shown in Figure 3-2.

Whereas the caller provides the name of the object when creating Tk widgets (necessary since parenting information is embedded in the name), package classes generate the name of the object and return it to the caller via a "create" method. Thereafter, the object name is passed to class methods. Methods related to a class are localized in source files so they can be loaded as needed using the TCl auto_load feature. Use of the class attribute array limits the reusability of individual procedures to within its package, a weakness inherent in this approach.

\subsubsection{Example}

We used the class package concept for developing a graphical front end for SQL-based databases. Four data display constructs were implemented as packages:

- BarWindow

- SpreadSheet

- SpreadWindow

- TextWindow

A SpreadWindow contains a SpreadSheet, so resuse is already evident. All the packages are designed to be usable in other applications as well. Tcl/Tk source for the application is listed in Appendix A. We examine Barwindow. tcl below.

\section{Specification}

Since there are no explicit constructs for exporting or importing individual procedures or declaring variables. we must rely on documentation. Further, we cannot separate specification and implementation. Thus, the file header (shown in Figure 3-3) identifies which procedures are considered "exported." Next in the file, we set default resource values via the option command. These resources may be overridden in .Xdefaults files or by executing xrab. Specification concludes with a comment block "declaring" object attributes. Of course, specifications of the procedure interfaces are found where the procedures are defined.

\section{$\underline{\text { Implementation }}$}

Procedure/method bodies begin with the global command to identify the class array variable. Adhering to the software engineering principles of high intramodule cohesion and low intermodule coupling as much as possible, we reference only the class attribute array and procedure parameters.

Figure 3-4 lists Barwindow_create, the package factory procedure. The new object's name is generated and retumed to the caller and serves as the caller's handle for referencing the object via package procedures. Windows are constructed using other package methods, BarWindow_CreateMenuBar and Barwindow_CreateGraph.

Importing the Package 


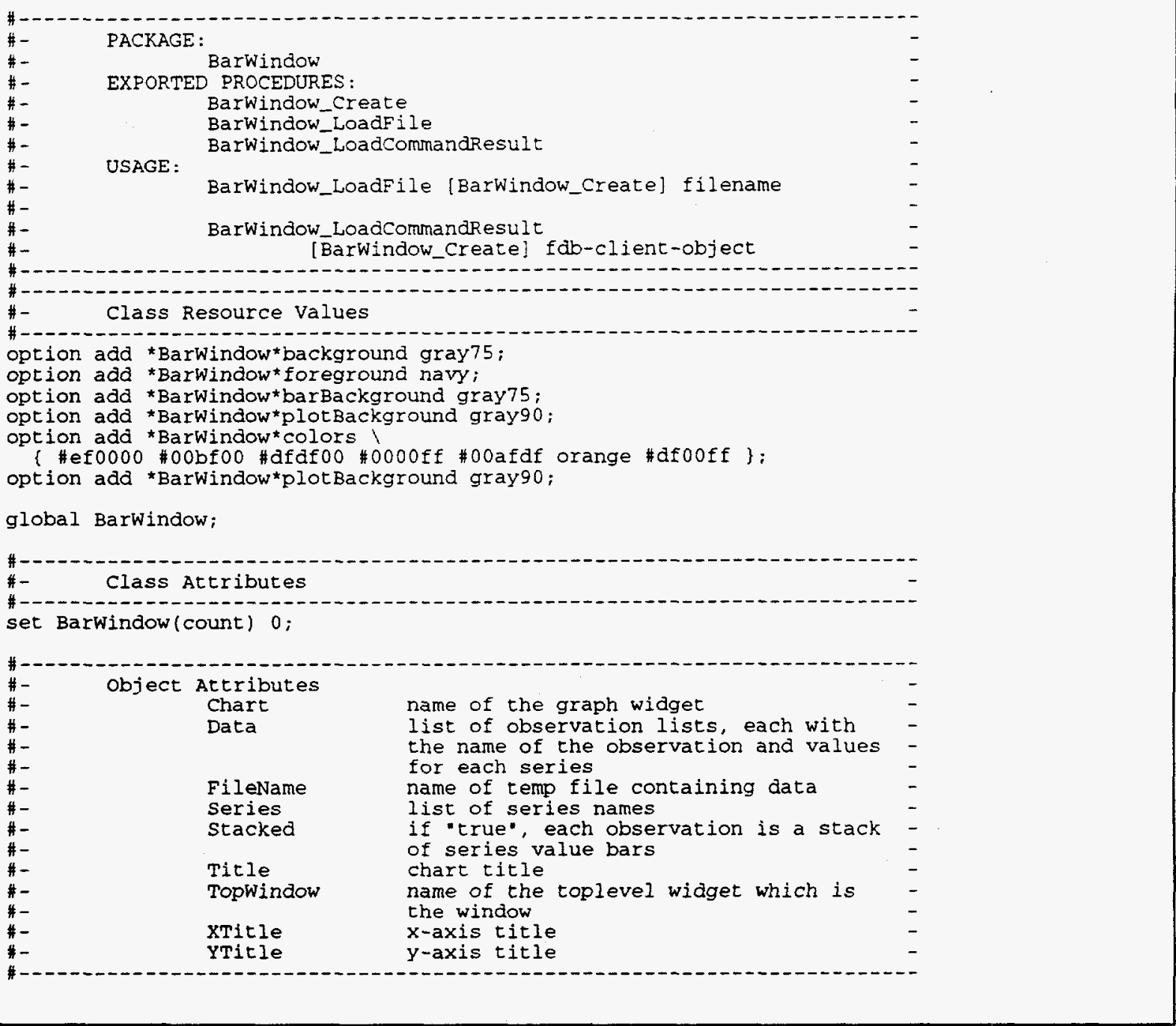

Figure 3-3 Tcl/Tk Package Specification

Driving the application is a module which builds the application menu bar and main window (Fab.tcl). Since there are no package import language constructs, the driver must explicitly set a package's necessary resource values. However, when a procedure is

option add *BarWindow*library \$Fdb_library;

option add *SpreadWindow*Iibrary \$Fdb_library;

option add *TextWindow*library \$Fdb_library;

\section{Figure 3-5 Setting the Library Resource to Import Packages}

referenced, its file location, if not in the current file, is searched automatically. Before importing one of these packages, its library resource value must be set, as shown in Figure 3- 


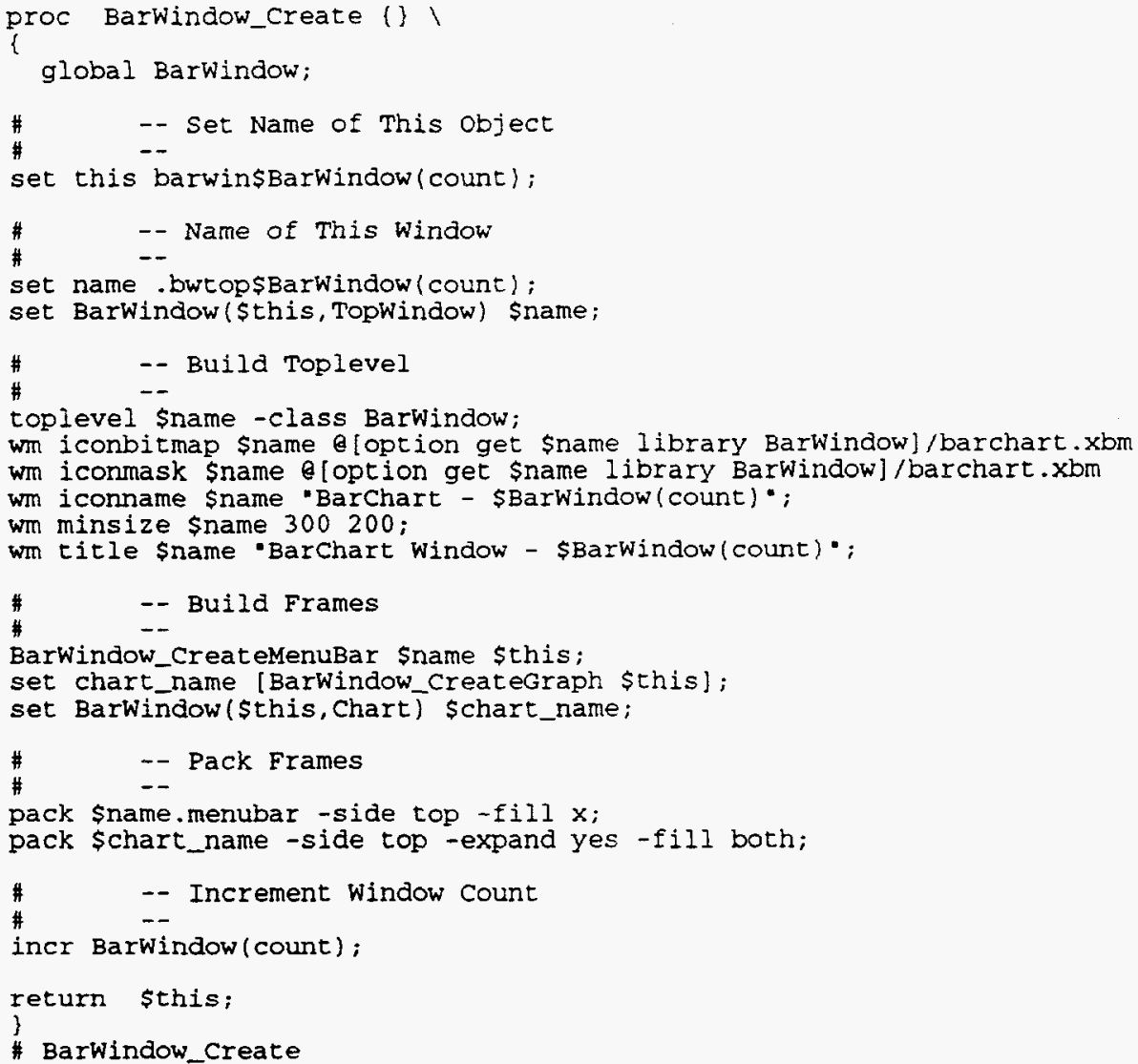

\section{Figure 3-4 Package Factory Method}

5. This option value points to the directory where run-time support files (e.g., icon bitmaps) are to be found. Each of the four packages can and hopefully will be used again in future applications. Although import is explicit, it is very simple.

Object-based packaging does provide some structure for development of reusable Tcl/Tk modules. However, the lack of support for inheritance hinders design and restricts reusability. Without some development framework, implementation in Tcl moves quickly toward an unmanageable heap of procedures that are very difficult to maintain. Development within the $\mathrm{Tcl} / \mathrm{Tk}$ language would be greatly enhanced with extensions, implemented in $\mathrm{C} / \mathrm{C}++$, for declaration, definition, export, and import of class packages. 


\section{SUMMARY}

As a language abstraction, Tcl offers compelling power through list processing. As a rapid prototyping tool, $\mathrm{Tcl}$ is unequaled in the speed with which a functional, working Motif ${ }^{\mathrm{TM}}$ style GUI application can be implemented.

However, as a software development environment, Tcl provides too little support for enforcing design structure. A means for declaring variables and ensuring they exist when referenced is necessary. Other semantic processing, such as type checking, would benefit the language greatly. Whereas compilers will often take care of syntax and semantic problems, Tcl code must be exhaustively exercised at run time in order to find these errors as well as logic problems. A reasonable heuristic is to limit implementation in $\mathrm{Tcl}$ language to GUI objects alone. 


\section{EXTENDING THE WIDGET SET}

Extendibility is arguably TclTk's best feature for the developer. Functions and objects may be added to the language as available commands. In this section we focus on extending the widget set and drawing objects of Tk. Three types of enhancements are described: adding widgets, adding canvas items, and modifications affecting multiple widgets.

\subsection{ADDING WIDGETS}

Adding widgets is a refreshingly simple task in Tcl/Tk. Requirements for widget implementations are identified in Section 2.3. For each widget type, there exists a structure or class specifying the values/attributes stored for each object instance. Use of $\mathrm{C}_{++}$allows the specification (and modular manipulation of) class attributes as well. For an example, refer to the source files TkThing. $h$ and TkThing. $\mathrm{CC}$ listed in Appendix B. They contain a $\mathrm{C}_{++}$

$\begin{array}{ll}\text { Tk_Window } & \text { tkwin; } \\ \text { Tcl_Interp * } & \text { tkInterp; } \\ \text { unsigned short } & \text { tkFlags; } \\ \text { int } & \text { tkwidth; } \\ \text { int } & \text { kHeight; } \\ \text { int } & \text { tkBorderwidth; } \\ \text { Tk_3DBorder } & \text { tkBgBorder; } \\ \text { Tk_3DBorder } & \text { tkFgBorder; } \\ \text { int } & \text { tkRelief; }\end{array}$

Figure 4-1 Object Attributes for the TkThing Class

class declaration/specification and implementation/body, respectively, for thing widgets. A thing widget is very simple--it merely draws a diagonal line from the top left comer to the bottom right corner. Figure 4-1 lists its object attributes.

All widgets should have the first three attributes defined, for they store pointers to the widget's window and interpreter data structures and a mask of flags used to store the state of the widget, respectively. Most widgets will have a variable graphic size, making it necessary to store width and height. The final four attributes deal with background and border colors when drawing the widget.

The Tk_3DBorder type is used for three-dimensional shading of regions. When creating a Tk_3DBorder object, the caller specifies a single color, and the TK library computes the corresponding illuminated and shaded colors $(140 \%$ and $60 \%$ of base color red, green, and blue values). When drawing a three-dimensional region, such as via the supplied Tk_Fill3DRectangle () function, one of three relief values is specified: raised, sunken, or flat. Most of the Tk widgets are three-dimensional and have relief as a configuration option. Use of these features is illustrated in TkThing: : Display (), shown in Figure 4-5. In the 
sections below we demonstrate the requirements for widgets as implemented for things.

\subsubsection{Configuring the Widget}

Configuration is the process of reading option values and setting the corresponding widget attributes accordingly. Fortunately, much of the grunt work involved in configuring a widget is handled by the Tk_Configurewidget () function, which is passed an array of Tk_ConfigSpec records. Each widget implementation should include a statically defined specification array. Whereas in $\mathrm{C}$ this array will probably have global scope (due to reference in more than one function), we can make it a class attribute in $\mathrm{C}++$. Observe the declaration

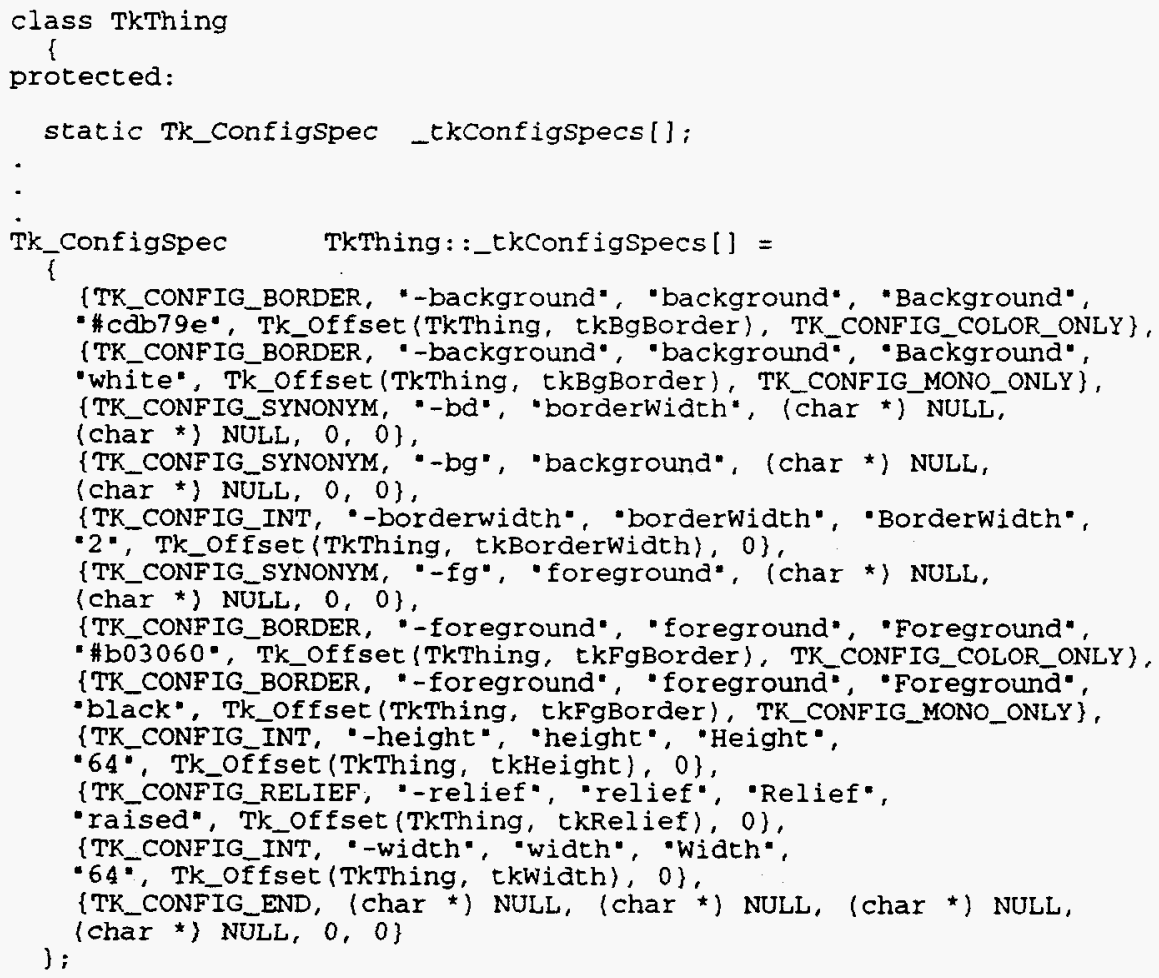

Figure 4-2 Widget Configuration $\mathrm{C}++$ Code

and definition of TkThing: :_tkConfigspecs shown in Figure 4-2.

A Tk_Configspec record describes the characteristics of the configuration option:

- the option data type

- switch name (beginning with a dash and used in scripts)

- option database name

- option database class name

- a default string value 
- the offset within the widget data structure of the corresponding attribute

- flag indicating the type of option

- optional function for storing and retrieving values for custom option types.

Offsets are computed using the Tk_offset macro, and flag macros are defined for most of the data types one can use (e.g., TK_CONFIG_BORDER for Tk_3DBorder attributes and TK_CONFIG_INT for int attributes).

Implementations for widgets with configurable options should support a configure subcommand for widget object commands. ${ }^{3}$ Configuration is also performed when the object is created. Figure 4-3 lists the TkThing: : Configure() method, which is modeled after

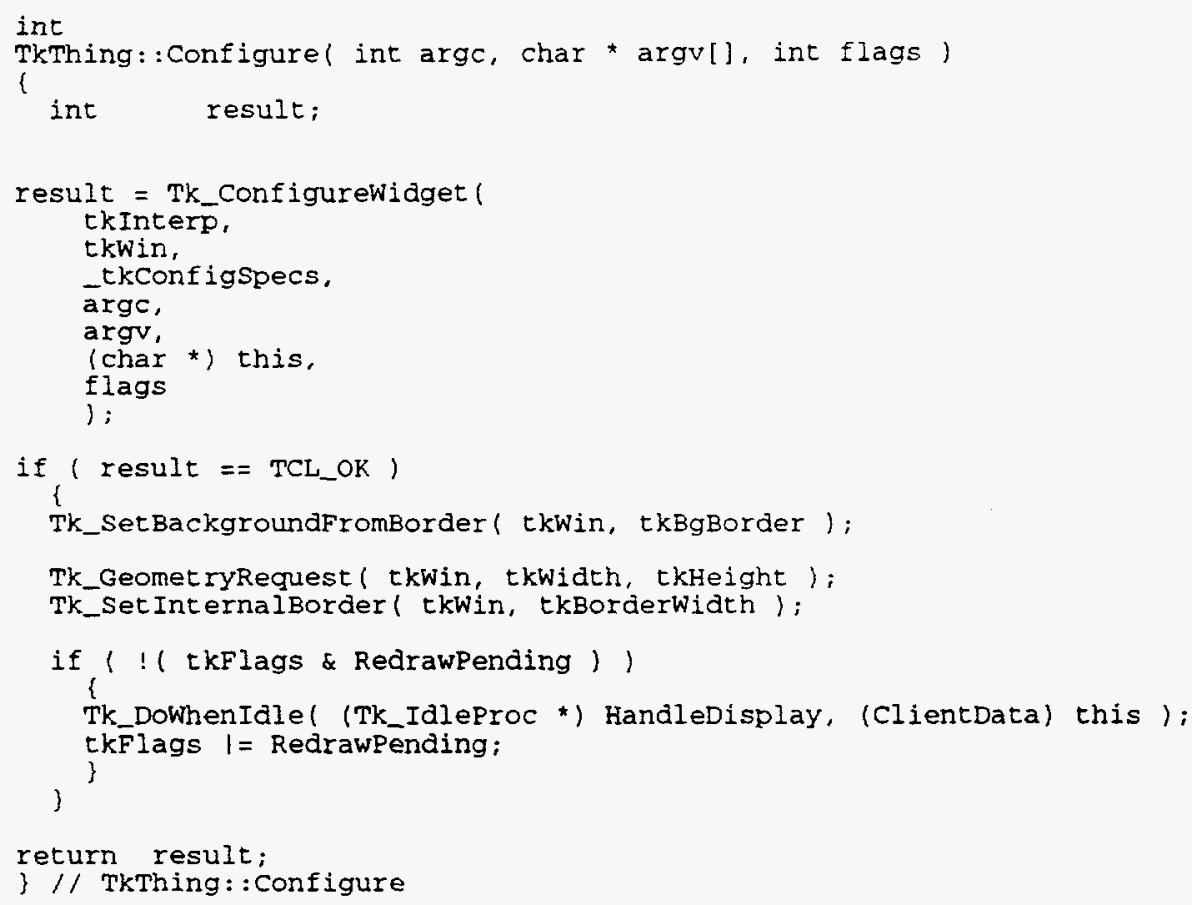

Figure 4-3 Widget Configuration C++ Method

widget configuration routines in the Tk distribution. Note that configuration may result in the need to redraw the widget object, in which case the RedrawPending flag bit is set and Tk_DoWhenIale () is called to register a class display method for invocation at a convenient time.

A subcommand is usually the first argument of an object command. 


\subsubsection{Handling $X$ Events}

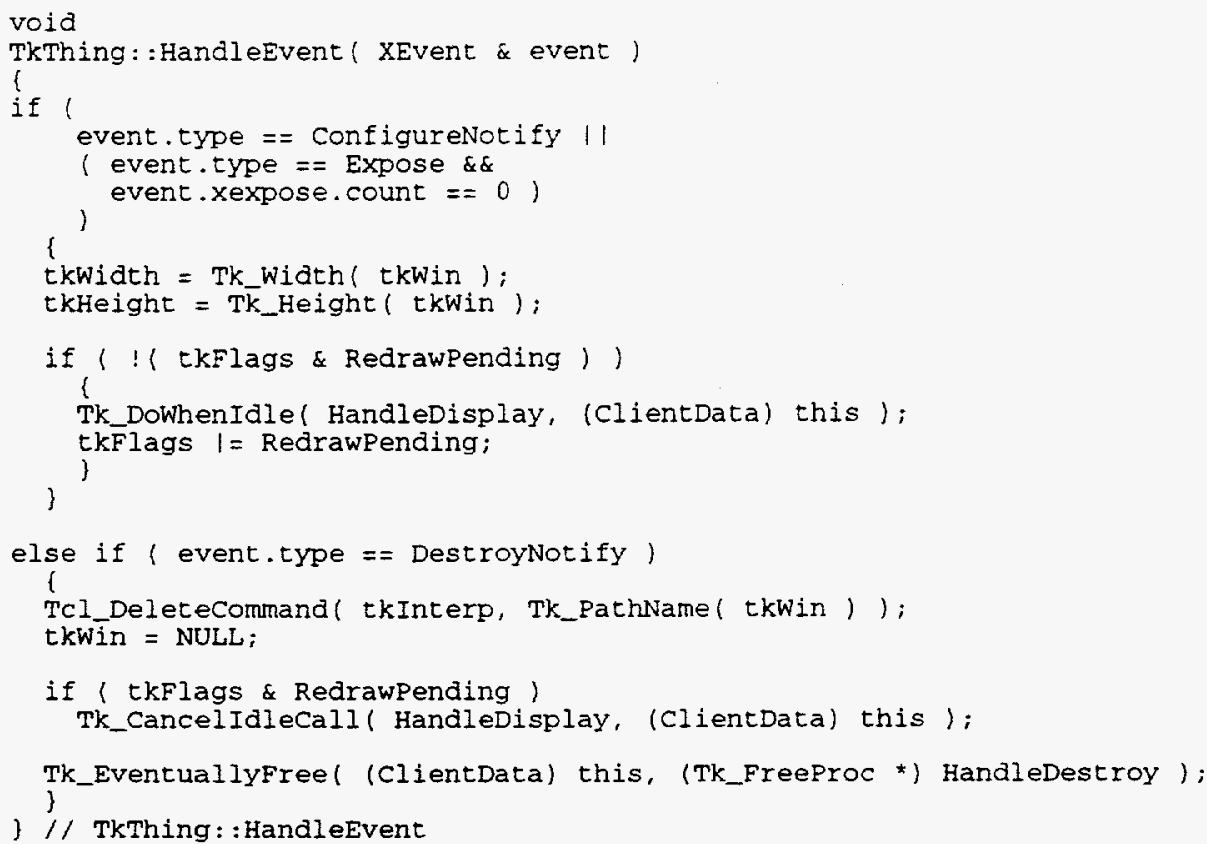

Figure 4-4 C++ Class Method for Handling X Events

The types of $X$ Window events to be handled for a widget are dependent upon the nature of the widget, of course. Highly interactive widgets must respond to appropriate events (e.g., ButtonPress, MotionNotify). Resizable widgets must handle configurenot ify, but all widgets should process Expose and DestroyNotify. Thing widgets are not interactive, but the class method TkThing: : HandleEvent (), listed in Figure 4-4, shows the algorithm for drawing a widget. Since things are resizable, we may need to draw on Configurenotify as well as Expose events. (We draw the entire widget rather than trying to reconstruct occluded regions, so we wait for an expose count of 0 .)

Tk provides a mechanism to improve overall performance with Expose events. A call to Tk_DowhenIdle () requests that a function (or $\mathrm{C}++$ static method) be invoked when little other processing is ongoing. The RedrawPending mask bit is cleared when the widget is drawn in TkThing: : Display ().

\subsubsection{Displaying the Widget}

Since display of a window is requested by the $X$ server via an Expose event, the event handler method initiates most drawing of the widget. Some configuration changes require update of a widget's appearance. The static class method TkThing: : HandleDisplay (), which merely invokes Display () for the object, is necessary so that a function address may 


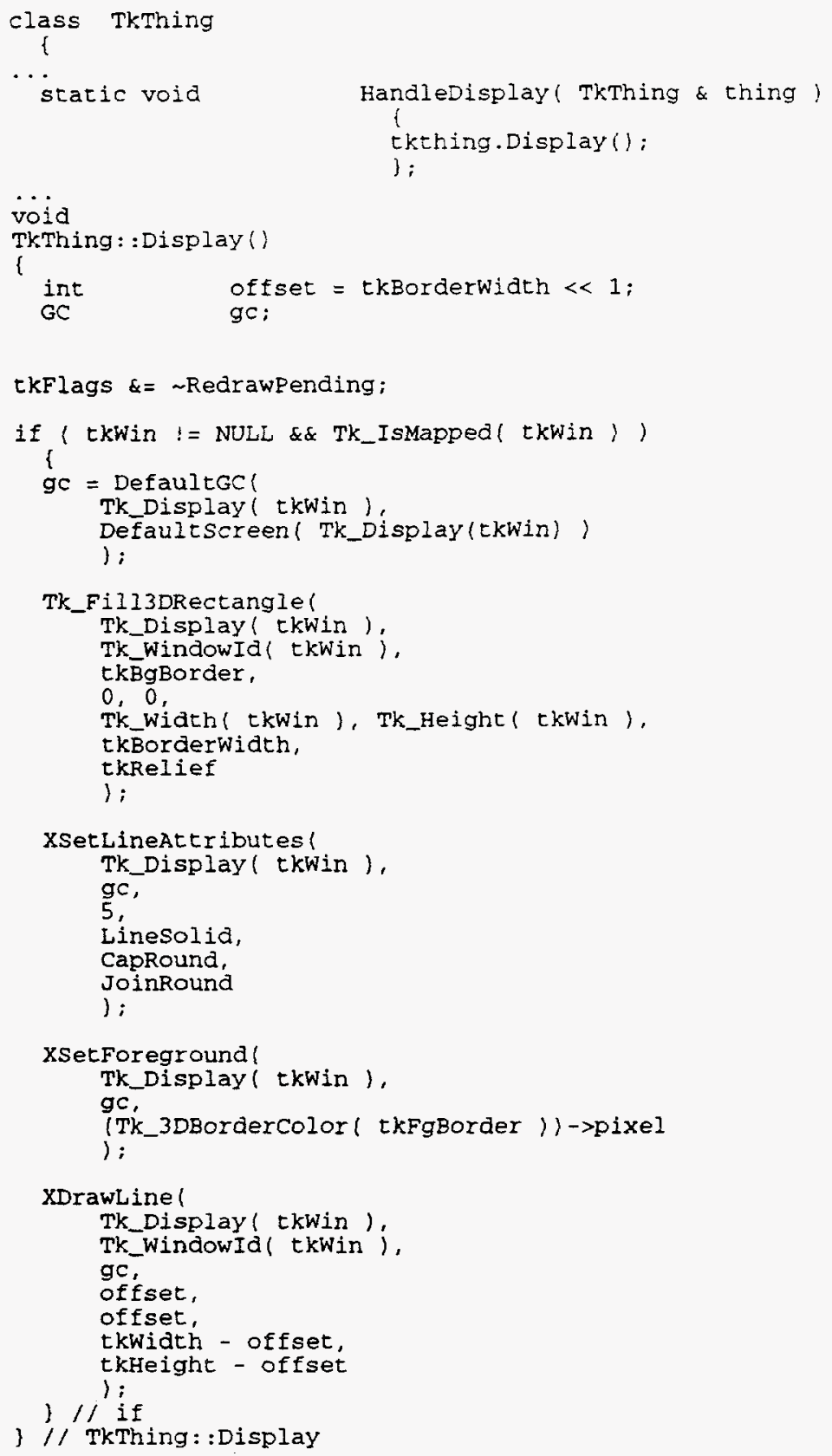

Figure 4-5 Widget Display Code

be passed to Tk_DowhenIdle (). Figure 4-5 lists the two methods. ${ }^{4}$

4 The client data value passed in the Tk_DoWhenldle() call is the C++ object address, which is passed by TclTk to the invoked routine. This address can be declared as a reference in the called method. 
Widget display is accomplished using Xlib and Tk drawing functions. $X$ context information (display, screen, drawable, graphics context) can be obtained from the tkwin object attribute (class member).

\subsubsection{Destroying the Widget}

A DestroyNot ify event instigates widget destruction. The $\mathrm{Tcl}$ command for the widget must be unregistered, any pending display calls must be canceled, any memory allocated for the widget must be freed, and the widget data structure itself must be freed. This presents some potential problems. In the parent-child widget tree, it is possible for a child widget data structure to be deallocated prior to reference within a parent. Tk provides the Tk_Eventual IyFree () function to circumvent this problem. It delays a widget's deallocation until all its ancestors have destroyed themselves.

Note also that our use of $\mathrm{C}_{++}+$requires another static class method, TkThing: : HandleDestroy ( ), which is passed to Tk_EventuallyFree (). The widget object is deallocated with the $\mathrm{C}++$ delete operator.

\subsubsection{Responding to Widget Commands}

As with options, the nature of the widget determines what commands it will support. A good deal of design effort should go into the subcommands a widget will process. In addition to configure, which all widgets should provide, widgets representing a value should provide subcommands for storing and retrieving values (e.g., set and get).

TkThing: : Processcommand (), the object method for handling the thing commands, is called from the static class method TkThing: : Processwidgetcommand () (refer to Figure 4-6). Here the only subcommand is configure, but it must be handled specially due to its three forms:

- no additional arguments -- request the values of all options

- one additional argument -- request the value of an option

- two or more additional arguments -- set the values of one or more options

Tk_ConfigureInfo () builds return value strings for the first two forms. Refer to Figure 4-6 for a widget object command handler.

\subsubsection{Creating Widget Objects}

Widget objects are created via a factory command. In our example, the command is thing, the implementation for which is the class method TkThing: : ProcessFactorycommand(), listed in Figure 4-7. Tk_CreatewindowFromPath() handles creation of the widget's $X$ window from the window path name. A new $\mathrm{C}_{++}$object is allocated with the new operator, which calls the appropriate object constructor for initialization. The widget's class name (for the option database operations) is set, the $X$ event handler for the window is registered, and a command for the new widget object is registered. 


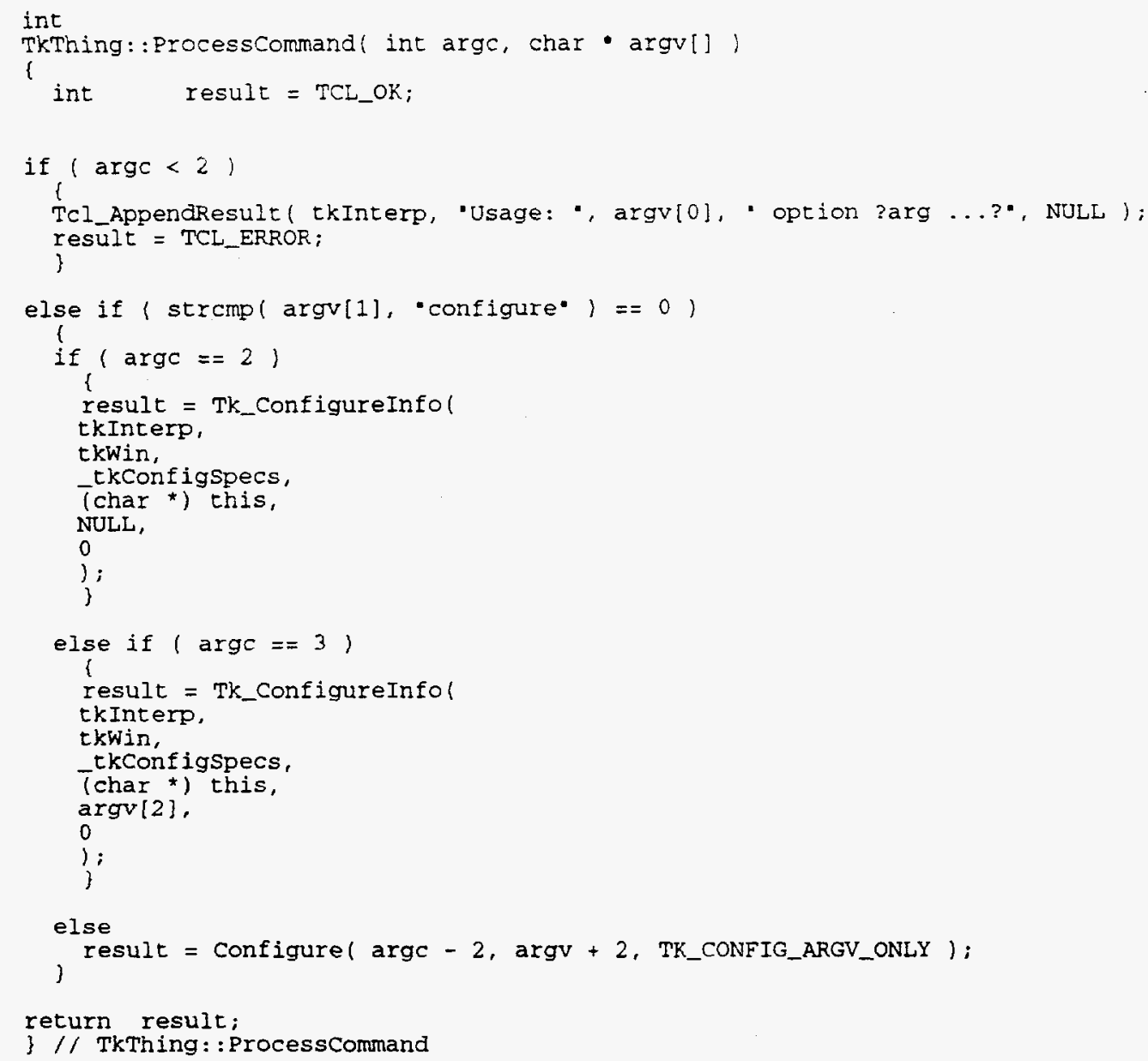

Figure 4-6 Widget Object Command Handler

\subsection{ADDING CANVAS ITEMS}

Unfortunately, the ease of adding widgets to TclTK is not quite duplicated for canvas items, though there is arguably less need for extension of canvas item types. Canvas items are not windows, but they are otherwise very similar to widgets in that both must support configuration (using the same facilities), deletion, and both must draw themselves. Section 2.3 enumerates the requirements for canvas item type implementations. Of particular note are the distance computation and PostScript generation routines.

Appendix $\mathrm{C}$ contains the source code for a canvas item extension, the bar item, implemented as the $\mathrm{C}++$ TkBarItem class. TkBarItem contains two class attributes: a Tk_Configspec object describing configuration options, and a Tk_ItemType object, necessary for canvas item implementations. It gives the canvas widget information necessary for processing the item type, including: 


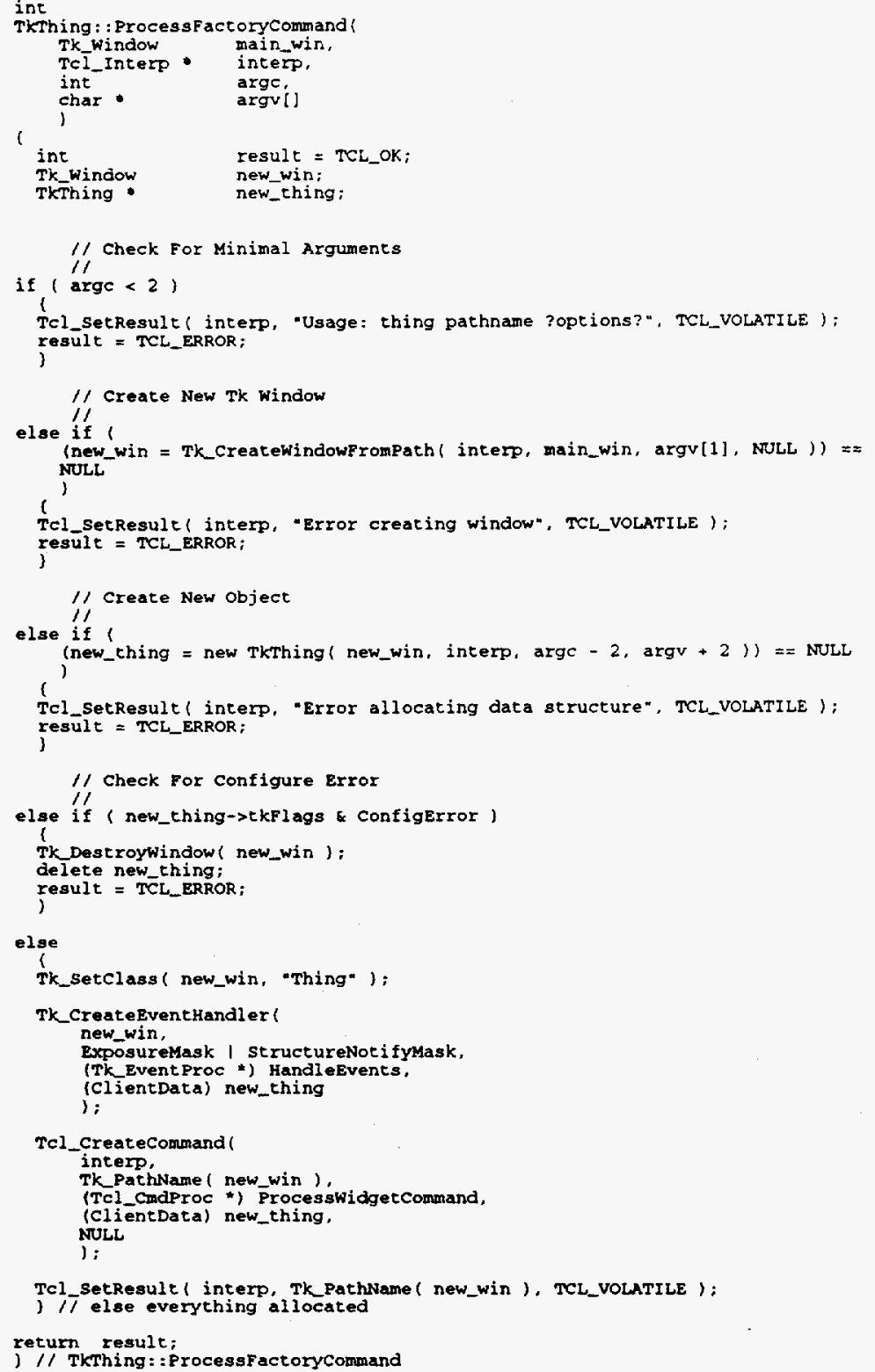

Figure 4-7 Widget Factory Command Handler

- the item type name,

- the size of the item's data structure, and

- the addresses of required and optional routines

Item types are registered by passing the Tk_ItemType object to the Tk_CreateItemType () function. Figure 4-8 shows the definition of the two class attributes. 


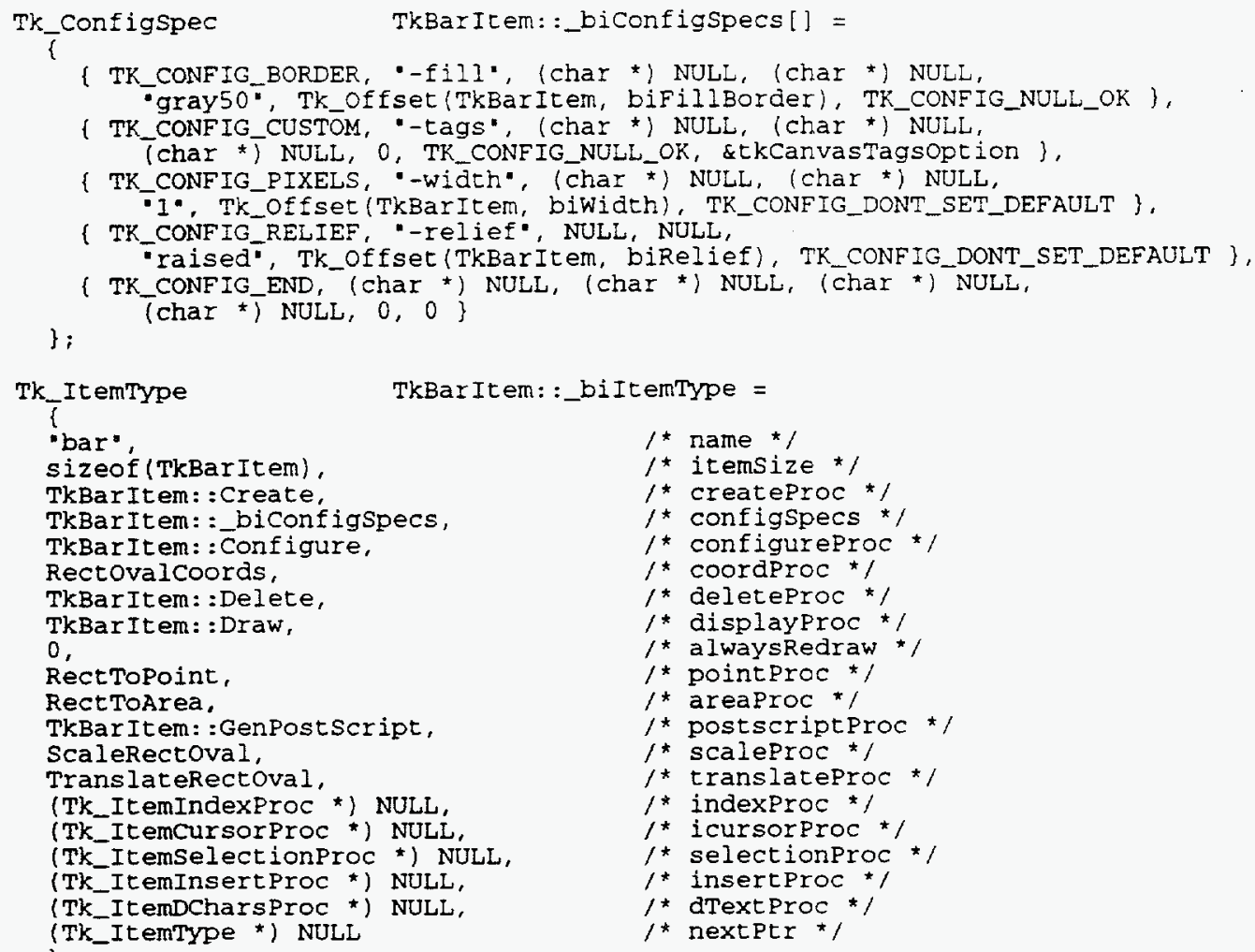

\section{Figure 4-8 Canvas Item Configuration and Type Definition}

\subsubsection{Creating an Item Instance}

Canvas item types are created via the canvas create subcommand, so no factory command is necessary. In our example, the creation is implemented with the class method TkBarItem: : Create (), listed in Figure 4-9. The canvas allocates the item object's memory prior to calling the create function. As with widgets, initial configuration must be performed, and the initial coordinates passed as command arguments are set using the TkGetCanvasCoord() function.

\subsubsection{Item Configuration}

Canvas item configuration mirrors widget configuration with one exception, the need to update any coordinate changes. After calling Tk_Configurewidget (), the class method TkBarItem: : Configure () calls ComputeRectOvalBox () to update bounding box information, as illustrated in Figure 4-10.

\subsubsection{Storing and Retrieving Coordinates}

Extent or boundary information must be maintained for a canvas item for drawing, 


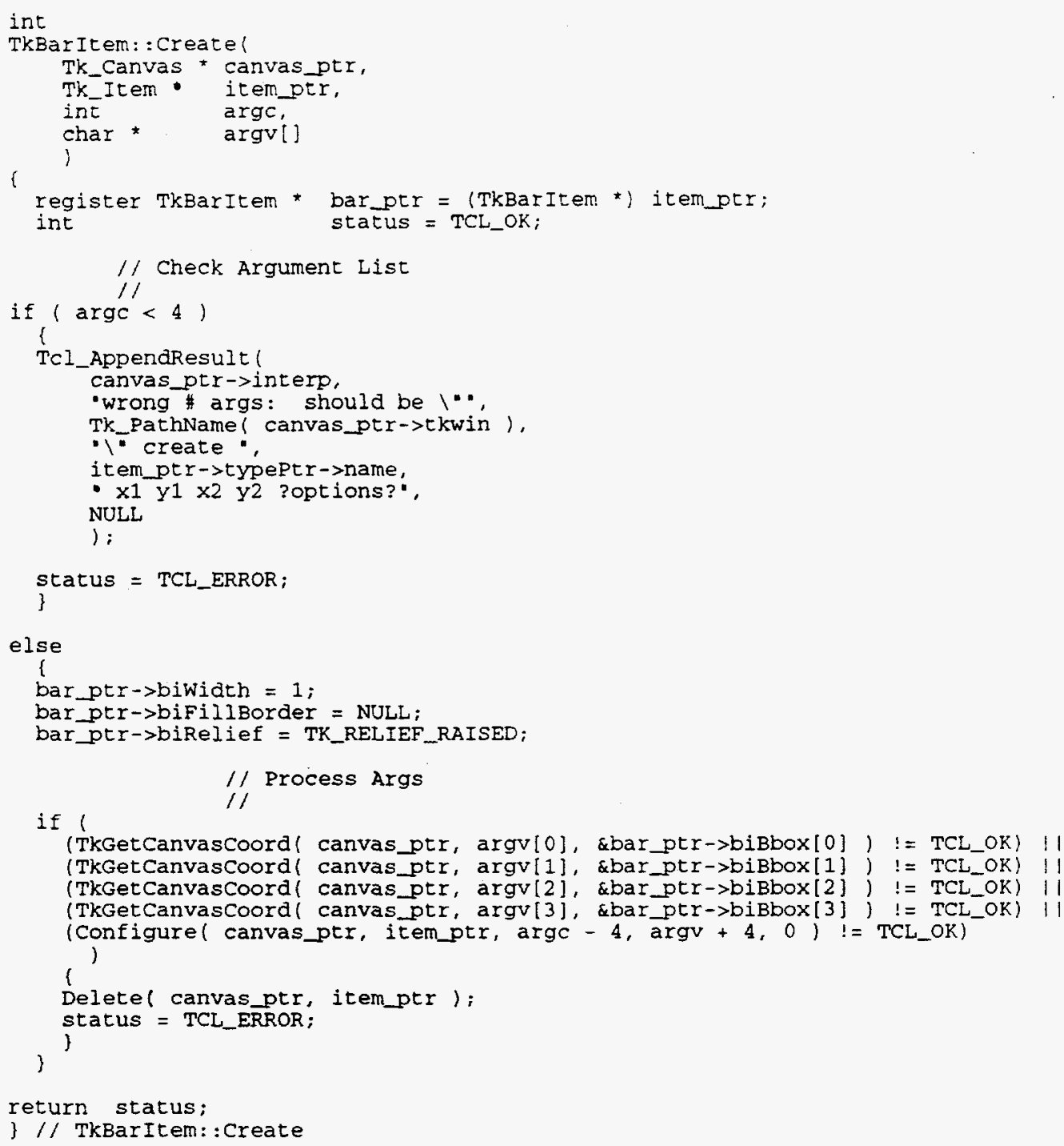

Figure 4-9 Canvas Item Create Method

computing distances to points, and determining overlap with regions. Canvas item types native to Tk include rectangles, ovals, polygons, and text, so most geometric situations are already handled within Tk source code. However, these $C$ routines, internal to the Tk library, are defined as static, and in order to make them exportable, and thus usable, modification of the Tk source is necessary. The bar item type is rectangular in shape, so we removed static tags and used the routines from tkRectoval.c. RectOvalcoords () is registered to handle maintenance of the item's bounding box.

\subsubsection{Deletion}

Obviously, when a canvas item is deleted, any memory or objects allocated during the 


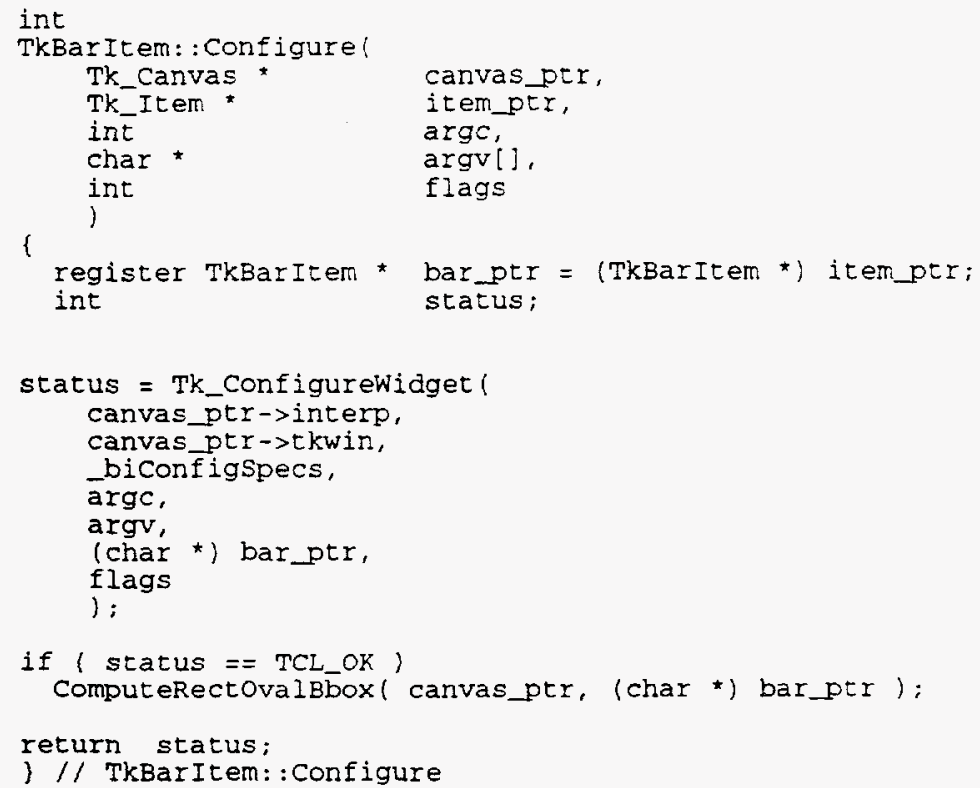

Figure 4-10 Canvas Item Configuration

item's life must be freed. The canvas takes care of deallocating the memory for the item itself. Refer to the class method TkBarItem: : Delete().

\subsubsection{Drawing the Item}

Unlike widgets, canvas items are not themselves windows. Instead, they are drawn by the canvas in its widget window. Thus, item coordinates must be translated to the coordinate space of the canvas's current scroll region. TkBarItem: : Draw () is listed in Figure 4-11. Note that the canvas passes the $X$ Drawable for use with $X$ rendering commands. This is often a pixmap used for buffering canvas display.

\subsubsection{Distance Computation}

As with bounding box maintenance, the distance computation routines are taken from tkRectOval.c. RectToPoint () computes the distance from the item's extent to a point, and RectToArea () determines whether the item lies within, outside, or overlaps a rectangular region.

\subsubsection{Generating PostScript}

Canvases provide the postscript subcommand to generate a PostScript representation of the canvas. Thus, each canvas item type must be capable of rendering an item in PostScript. TkBarItem: : GenPostScript (), listed in Figure 4-12, demonstrates the PostScript generation for a canvas item. Again, Tk provides support routines. TkCanvPsY () projects the canvas coordinate into the vertical dimension of the PostScript page, and 


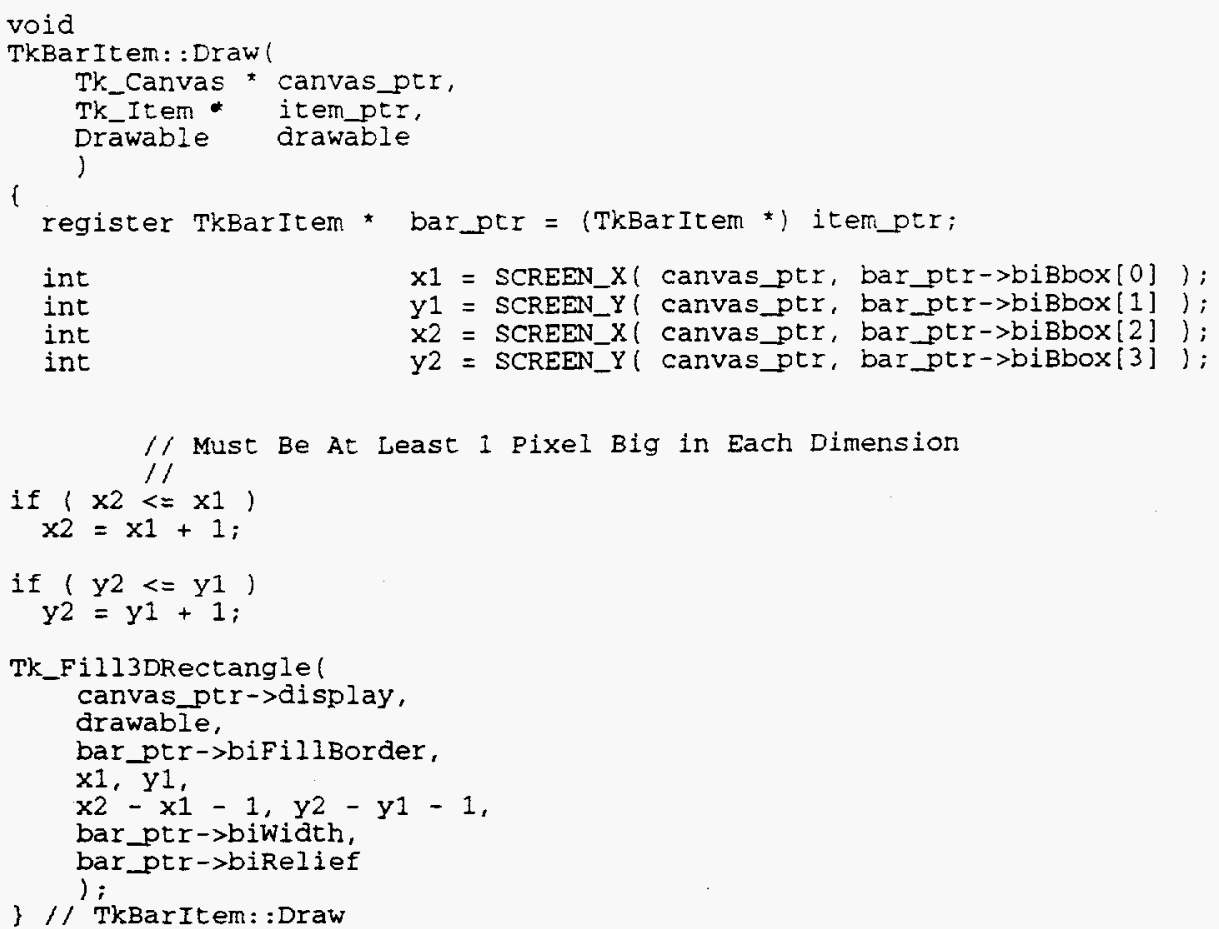

Figure 4-11 Drawing the Canvas Item

TkCanvPsColor () generates PostScript commands to set an appropriate color. PostScript commands are set as the interpreter result in the generator routine.

\subsubsection{Scaling and Translation}

In response to the scale and move subcommands, a canvas widget calls routines for referenced items to scale or translate, respectively. Both operations require update of an item's extent or bounding box. For the bar item we use ScaleRectoval () and TranslateRectoval (), respectively, from tkRectoval.c.

\subsection{OTHER ENHANCEMENTS}

Enhancements other than new widgets or canvas items will likely require modification of source in the Tk distribution. We describe two examples here: a simple enhancement of the Tk_3DBorder facilities, and extension of bitmap processing to include XPM2 pixmap files.

\subsubsection{Border Color Functions}

There are many data structures and functions defined for internal use within $T c l / T k$. The discussions above identified the need to export needed functions. Adhering to the software engineering principle of information hiding, many data structures have extemal or exported versions which are merely opaque pointers. Examples include: 


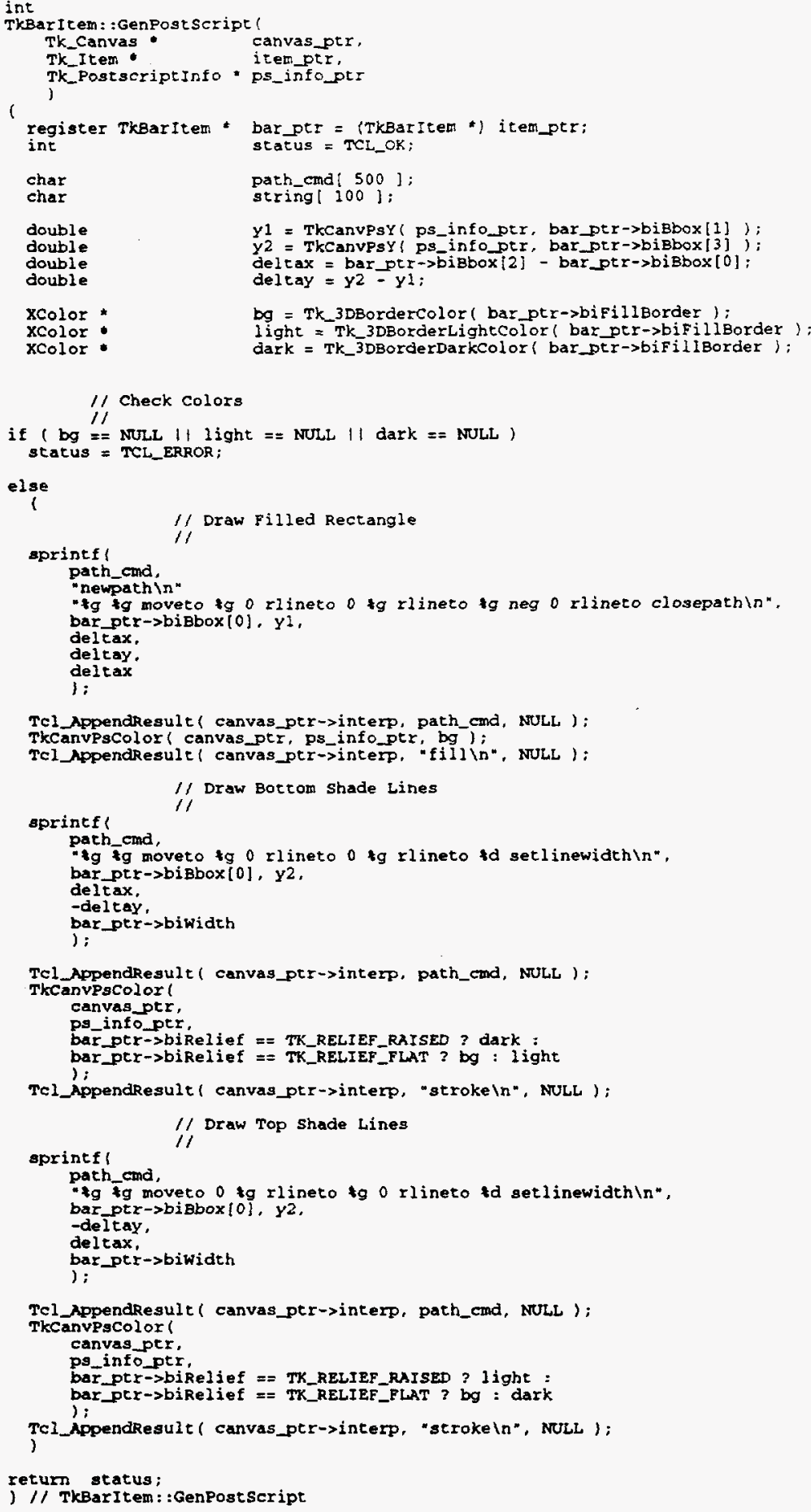

Figure 4-12 Canvas Item PostScript Generation

- private TkWindow and public Tk_Fakewin

- private Border and public Tk_3DBorder 


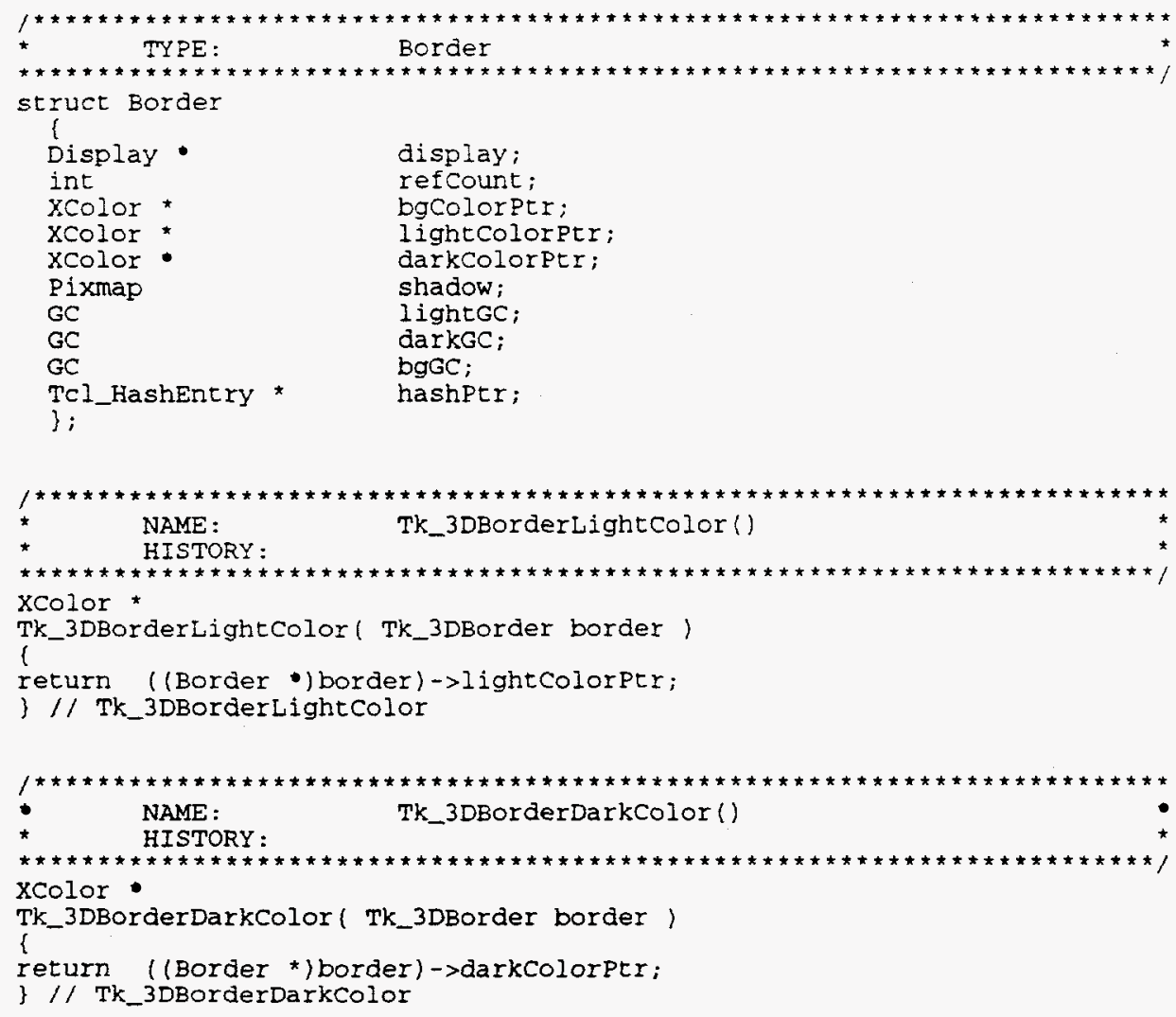

\section{Figure 4-13 Retrieval of Shade Colors From a Tk_3DBorder}

Border objects store XColor values for background, light shade, and dark shade colors. However, only the opaque pointer type $\mathrm{Tk}$ _3DBorder is exported. A function is provided to retrieve the background color from a border, Tk_3DBorderColor (), but no facilities are provided for retrieving the shade colors. Figure 4-13 lists the trivial code to support retrieval of the shade colors. The Border structure definition is repeated (not good from the maintenance point of view) to allow access of the necessary fields.

\subsubsection{Pixmap Processing}

Bitmap facilities are not effectively localized in an object package in Tk. Instead, they are spread among the various widget packages which process them:

- buttons,

- canvases,

- menus, and

- menubuttons.

Unfortunately, this requires modification of several Tk source files: 
- tkBitmap.c,

- tkButton.c,

- tkCanvBmap.c,

- tkMenu.c, and

- tkMenubutton.c

Tk extensions for handling XPM files exist, but at the time of our efforts there were no facilities for handling XPM2 pixmap files in the a format generated by iconedit under OpenWindows. We added this capability with two additional source files, tkPixmap. $h$ and $t k P i x m a p . c$, as well as slight modifications to the files listed above. Appendix $D$ contains the source listings. Tk supports reference of $X$ Window bitmap files (most commonly used as the value of the bitmap option) by name with a prefix of ' $a$ '. We extend this notation to include the 'os' prefix for XPM2 format files and the '^' prefix for $X$ Window dump files. Examples of such references follow:

- a/usr/include/X11/bitmaps/xlogo32

- $q /$ usr/openwin/include/pixmaps/folder.xpm

- ^/usr/local/xwd/picture.xwd

Two basic modifications were necessary to existing Tk code: changes within tkBitmap. $C$ to enhance bitmap processing, and changes to the widget source for rendering "bitmaps." Within tkBitmap. c, the unexported TkBitmap structure definition was modified to include a depth field, a Tk_DepthofBitmap () function was added for retrieval of a bitmap object's depth value, and the Tk_GetBitmap () function was enhanced to support the new prefixes in file name references.

As delivered, Tk widget sources render bitmaps using XCopyPlane (). Each such call had to be modified with a call to $T k$ _DepthofBitmap () to determine bitmap versus pixmap, and a conditional statement for rendering. Whereas a depth of one indicates a bitmap and results in an XCopyPlane () call, a depth greater than one is a pixmap and is rendered using $\mathrm{XCopy} P$ lane (). This is illustrated in Figure 4-14, which lists a code snippet from the DisplayButton () function in tkButton.c. 


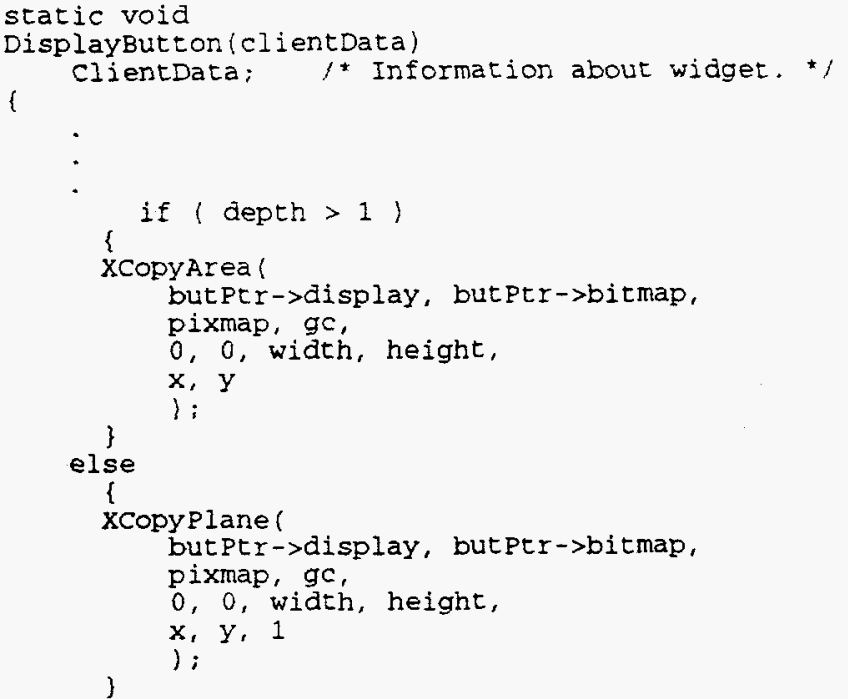

Figure 4-14 Code to Render Pixmaps and Bitmaps 


\section{EMBEDDING CLASSES AND FUNCTIONS}

One of the most practical ways to exploit the power of $\mathrm{TCl}$ is to build commands for classes, data structures, and functions developed in a $3 \mathrm{GL}$, thereby embedding their functionality within an interactive scripting language. This allows test harnesses to be scripted, permitting more exhaustive tests to be developed quickly. Further, one should work to improve the exportability of modules and classes in order to increase the likelihood of their reuse. Two examples of $\mathrm{Tcl}$ interfaces to $\mathrm{C}++$ classes are used to discuss the mechanics of such an interface and techniques for data exchange. The first, TclFabclient, is built on an application-specific socket communication facility. Second, Tclsampler fronts a statistical sampling facility. Source code for the examples is listed in Appendix E.

\subsection{TCL INTERFACES TO C++ CLASSES}

$\mathrm{C}_{++}$supports inheritance, the single characteristic distinguishing object-oriented from object-based environments [1]. Thus, we can construct a derived class which provides the boilerplate and processing methods for achieving a Tcl interface to the class from which it inherits. Figure 5-1 shows the declaration of such a class (with comments removed). Common to all such classes are object attributes, class methods for Tcl callbacks and registration, and object methods to implement subcommands. Clearly we want to use the object-based extension mode.

\subsubsection{Object Attributes}

A necessary object attribute for any $\mathrm{Tcl}$ class is a pointer to the $\mathrm{Tcl}$ interpreter, for it is passed to most Tcl/Tk functions. For object-based extensions, it is necessary to store the name of object instance as referenced in the Tcl script in order to unregister the object's command when it is destroyed. Hence we have the tfcInterp and $t$ fcName attributes, respectively. The first two parameters in the constructor are for setting their values. Thus, they must be provided when an object is instantiated.

\subsubsection{Class Methods for Tcl Callbacks and Registration}

C++ class examples in Section 4 demonstrated the need for class methods implementing the factory command, object command, and object deletion. In addition, the inline class method Register () calls TC1_CreateCommand () to register the factory command name, Fabclient, and the factory method, Processclasscommand().

Figure 5-2 lists TclFabclient: : ProcessclassCommand (). If the necessary arguments are provided, a new object is allocated and initialized via the $\mathrm{C}++$ new operator, which calls the constructor. If object allocation succeeds, TCl_createcommand () is invoked to register a command with the same name as the object itself. The callback for all object commands is ProcessobjectCommand (), and the object command deletion handler is Deleteobject ().

For consistency with other Tcl object-based extensions (e.g., Tk widgets), object 


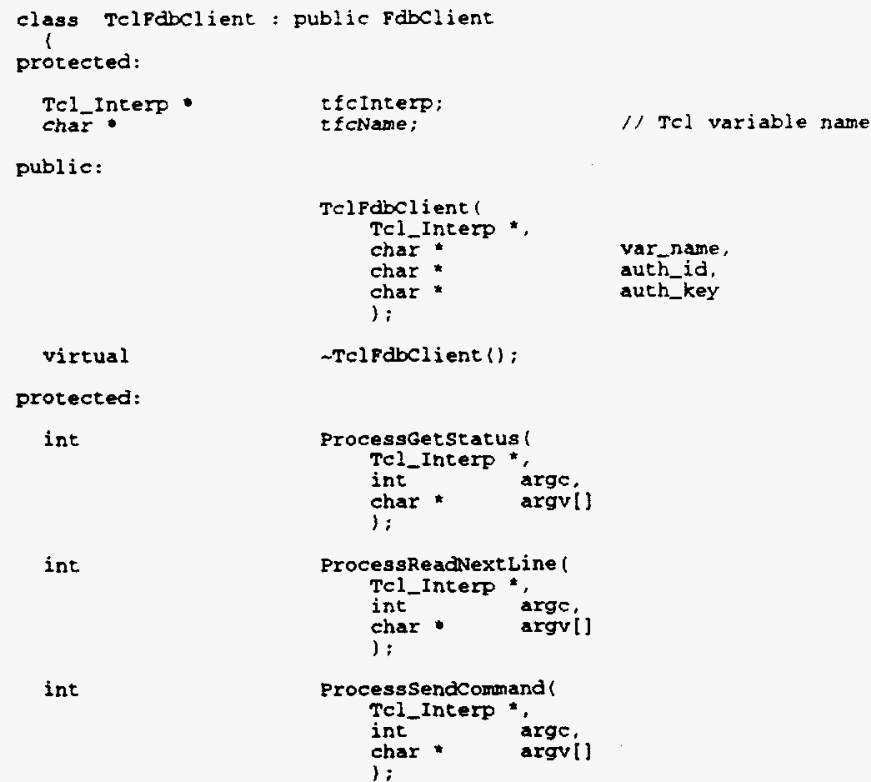

\section{Figure 5-1 Tcl Derived Class Declaration}

commands should take a subcommand as the first argument and subcommand-specific arguments and options on the remainder of the command line. In designing the command structure, we need only refer to the class from which we inherit. Methods to be interfaced should have a corresponding subcommand. In the case of TClFdbClient, we implement three such subcommands: GetStatus, ReadNext Line, and SendCommand. ${ }^{5}$ For each subcommand there is a corresponding object method. Tclpabclient : : Processobjectcomnand() is listed in Figure 5-3. When the subcommand is recognized as one of those supported, the

5 The use of capitalization in commands and subcommands is a departure from standard Tcl practice, but it identifies our local $\mathrm{C}++$ implementations and distinguishes them from other extensions. 


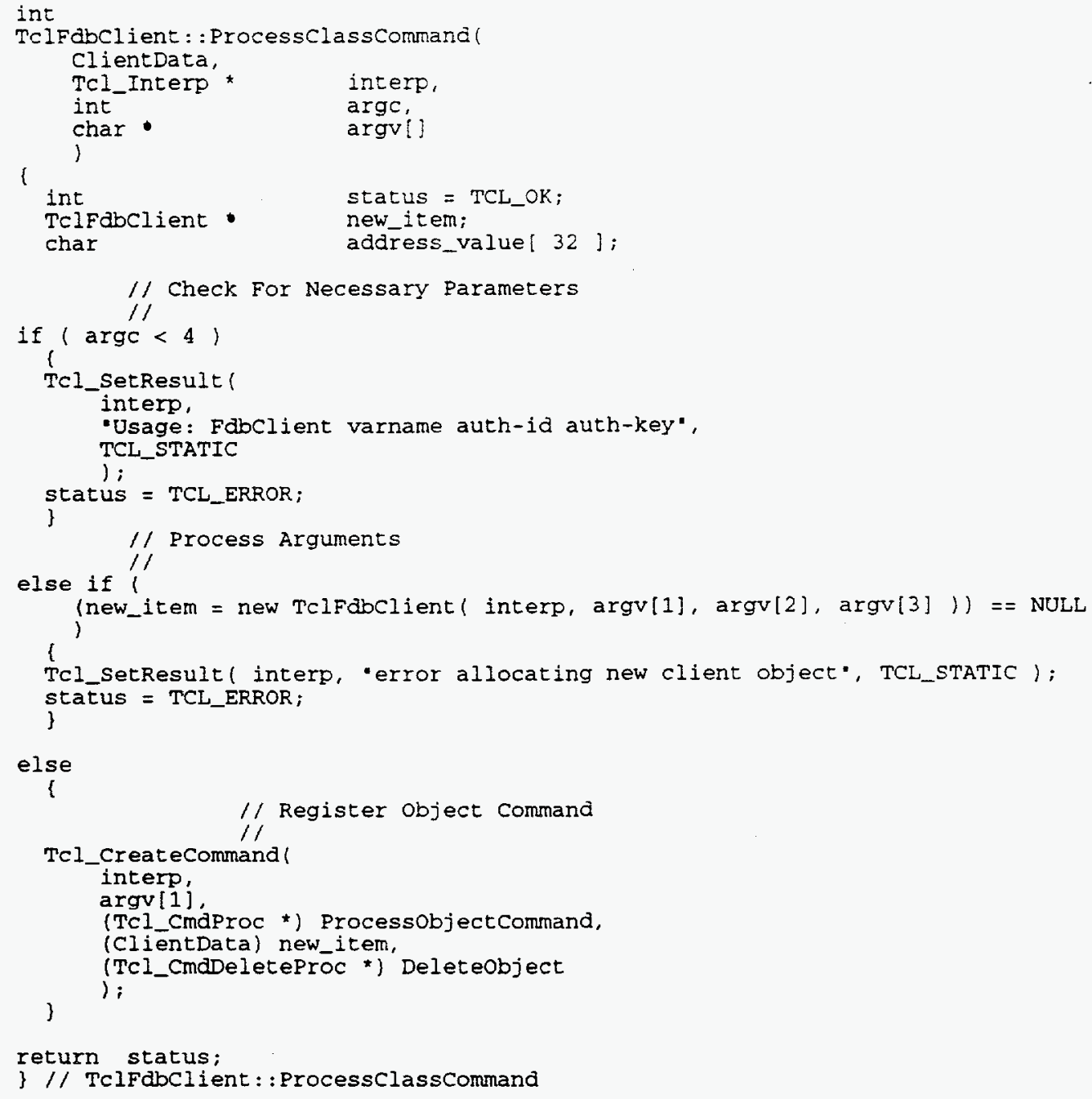

Figure 5-2 Tcl Class Factory Command Method

corresponding object method is invoked for the object. The remainder of the command line after the subcommand itself is passed to the subcommand method. Note that the Delete subcommand is common to all object-based commands and is handled with a call to TCl_DeleteCommand ().

For uniformity, one of our software engineering principles, the method naming scheme should be repeated for all $\mathrm{Tcl}$ interface classes:

- Processclasscommand() -- class factory command method

- Processobject Command() -- class object command method

- Deleteobject () -- class object deletion method

- Process???() -- object subcommand methods 


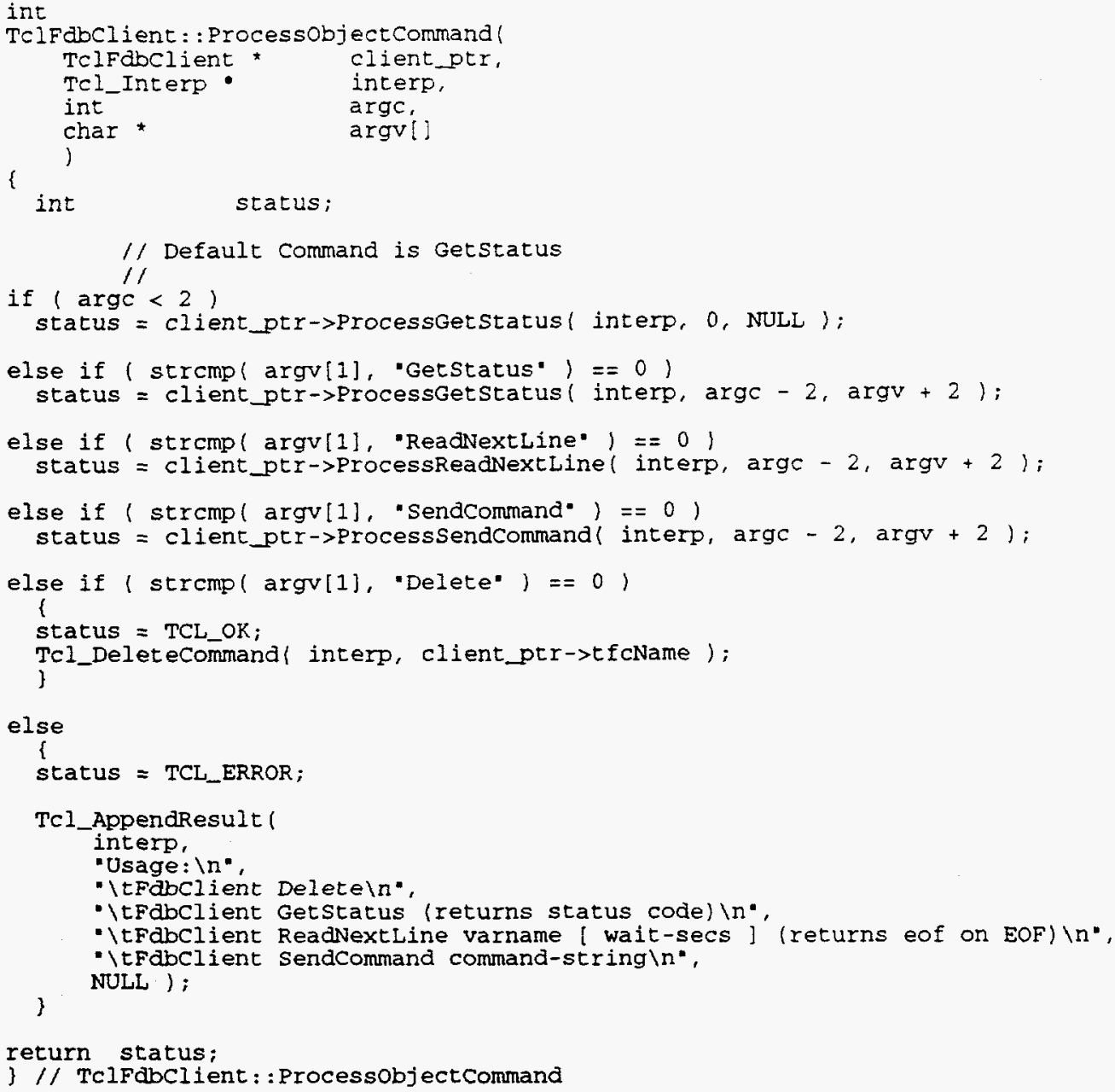

Figure 5-3 Tcl Class Object Command Method

\subsubsection{Object Subcommand Methods}

The specification for object subcommand methods contains three parameters: a pointer to the $\mathrm{TCl}$ interpreter, and argc and argv values describing the subcommand arguments. For an example, refer to Figure 5-4 which lists TclFdbClient : : ProcessReadNextLine (). Recall that all data are represented within Tcl as strings. As with any UNIX command, numeric arguments must be converted from string format. Within Tcl, numeric results must be converted back to strings. Command results are retumed by setting the result field of the $\mathrm{TCl}$ interpreter. Tcl provides five functions to assist in setting results:

- TCl_SetResult()

- Tcl_AppendResult ()

- Tcl_AppendElement ()

- Tcl_ResetResult() 


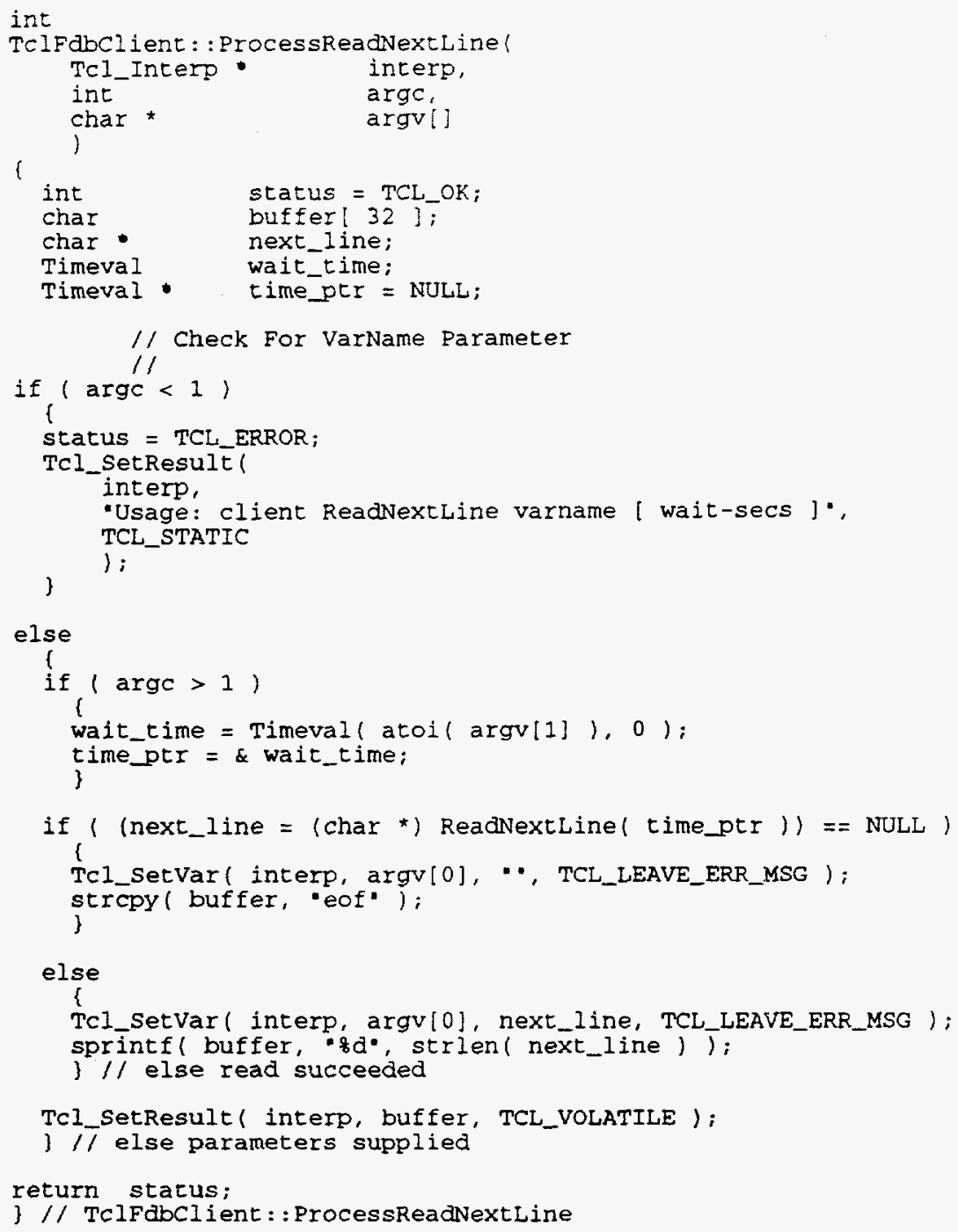

Figure 5-4 Tcl Object Subcommand Method

- Tcl_Freeresult()

ReadNextLine takes the name of a Tcl variable to set as an argument. Variable values may be set and retrieved via TCl_SetVar () and Tcl_GetVar (), respectively. Other functions are provided for setting and retrieving array values and removing a variable.

\subsection{TECHNIQUES FOR DATA EXCHANGE}

Inevitably, one will encounter a need to exchange a large amount of data, such as an array or structure, between the $\mathrm{Tcl}$ language and $3 \mathrm{GL}$ code. $\mathrm{Tcl}$ lists are an effective mechanism for this exchange. Further, addresses and handles may be passed as well. 


\subsubsection{Arrays and Lists}

Lists are the primary construct for storing collections of data in $\mathrm{TCl}$ and are implemented as strings. Elements are delimited within the string. Tcl provides several functions for manipulating lists, including TCl_SplitList () and TCl_Merge() for dividing a list into individual element strings and combining element strings into a list, respectively. If special handling is not required (e.g., processing backslash characters or nested quotes), lists can be created using string functions.

Figure 5-5 lists Tc1Sampler: : ProcessGetLastSample(). The GetLastSample subcommand of a Sampler object retums an array of tuples. We accomplish this by building a list of lists, which is merely a large string with the element lists inside braces. Within the for loop of Figure 5-5, sprint $f()$ is called to append the tuple lists to the outer list string. Figure 5-7 shows a sample Tcl subcommand call. Data arrays are passed in a similar way. Elements are extracted from the argument containing the list via TCI_SplitList ().

\subsubsection{Addresses and Handles}

The need may arise to exchange a memory address or data handle between $3 G L$ modules through the $\mathrm{Tcl}$ language. Very simply, these values may be exchanged as numbers in string form. An example of such an operation is in Tclsampler: : ProcessGet ImageAddr (), listed in Figure 5-6. The value of the sImage attribute, an XImage address, is set as the

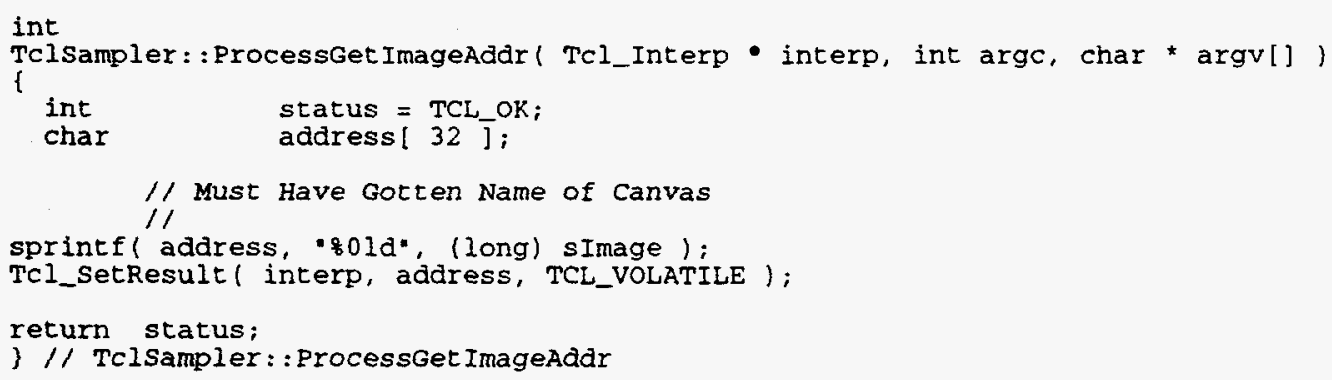

Figure 5-6 Returning a Memory Address in a Tcl Object Method

command result.

Figure 5-7 contains a Tcl script snippet invoking the Get ImageAddr subcommand. The resulting string address is processed as any other value resulting from a $\mathrm{Tcl}$ command. Figure 5-8 shows configuration data structure and method for the TkImageItem which receives the image address as the-address option value. 


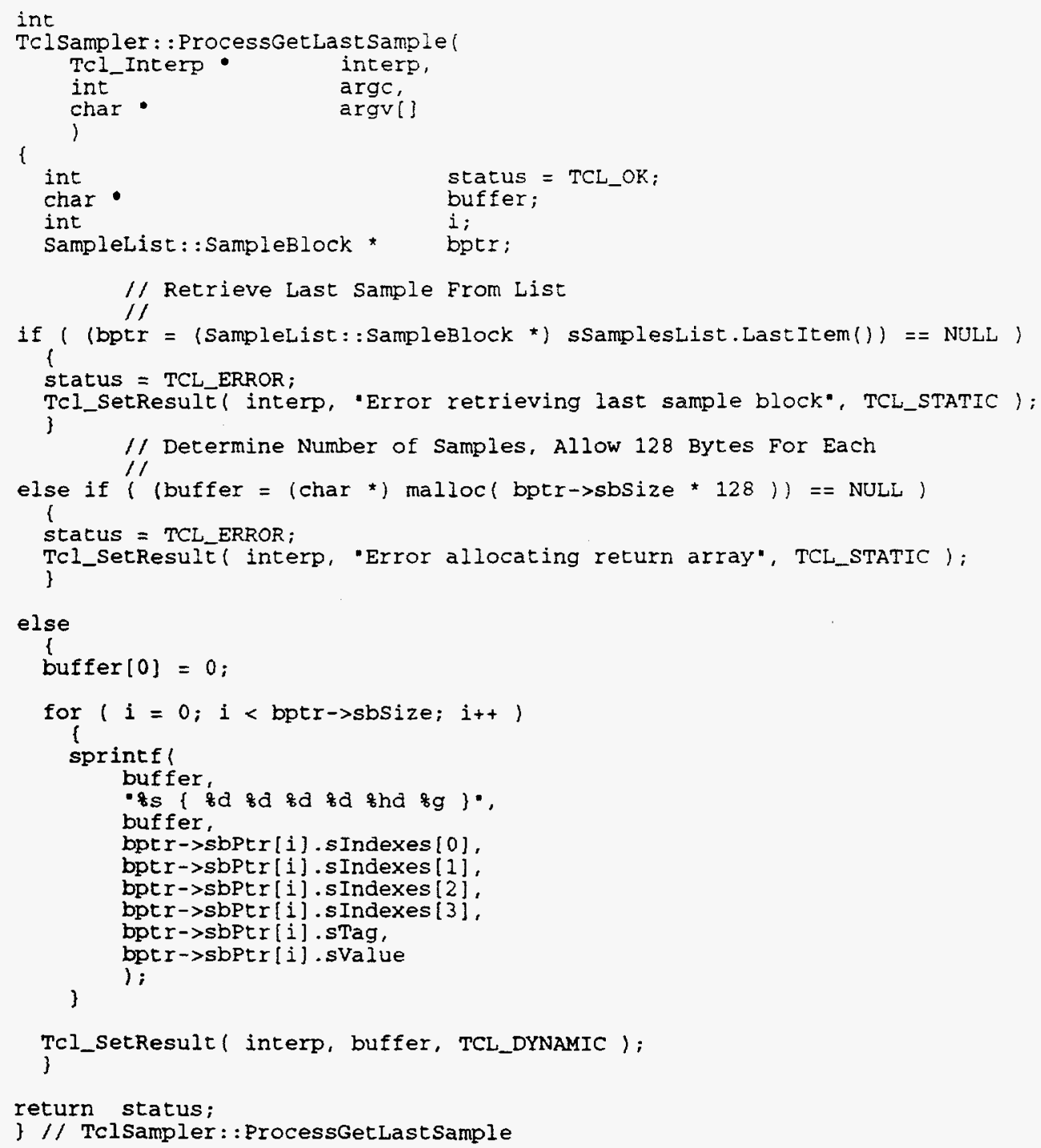

Figure 5-5 Return of Array Data in Tcl Object Method 


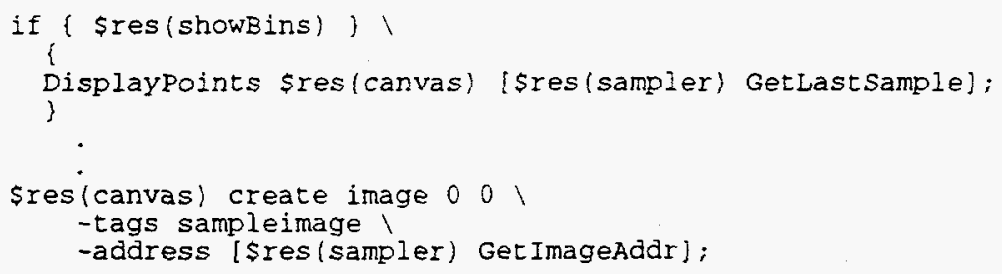

\section{Figure 5-7 Tcl Code Snippets}

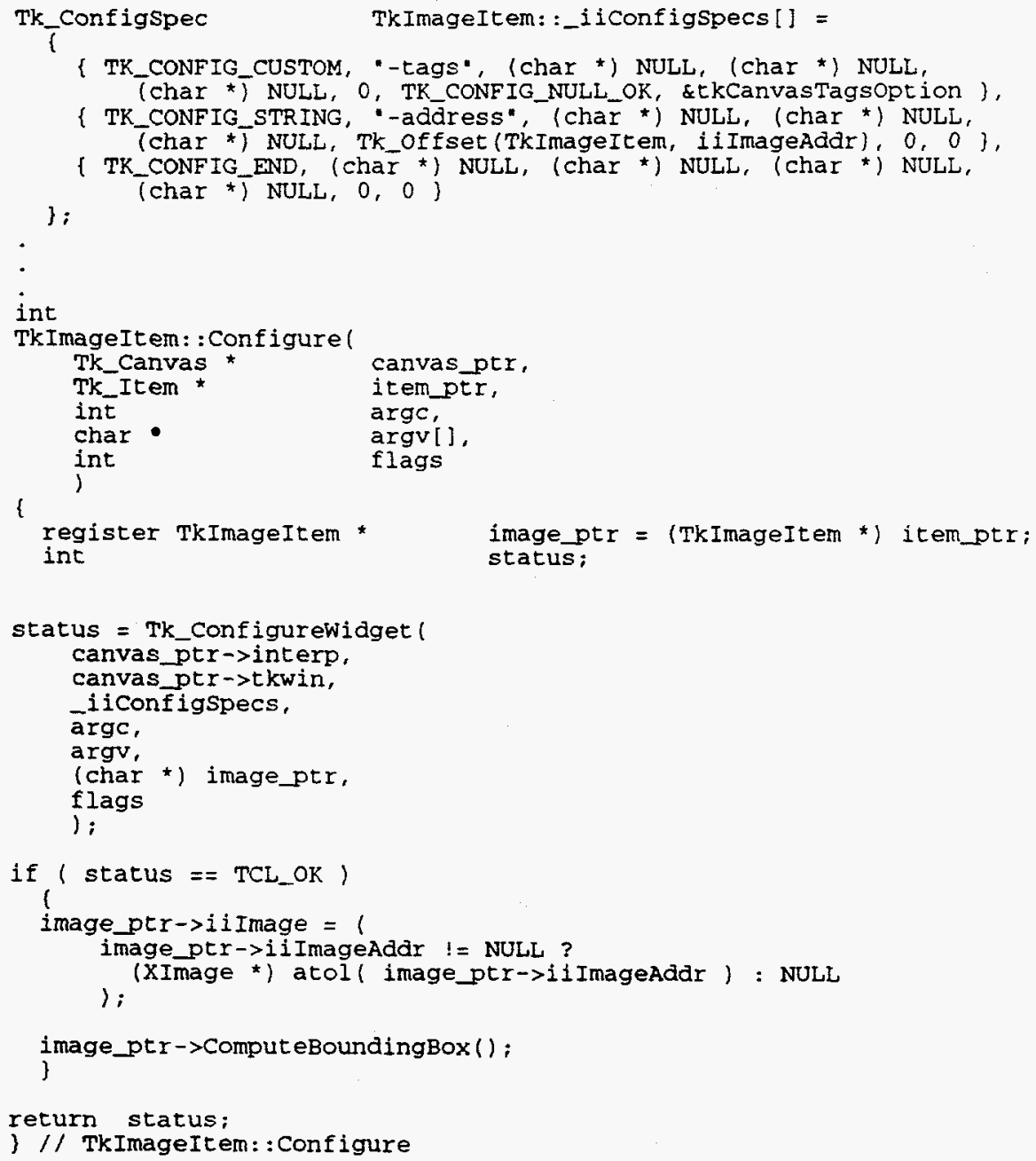

Figure 5-8 Configuration for Processing a Memory Address 


\section{COPROCESSES}

Tcl/Tk provides a very effective means for quickly generating an $X$ Window interface with interactive widgets such as push buttons, text fields, and radio buttons. Graphics can be generated rather quickly as well with canvas widgets. However, graphics requiring a lot of operations (e.g., drawing thousands of dots) can suffer from poor performance with command interpretation in Tcl. Intensive graphic applications, such as image manipulations, using Xlib calls will all but require implementation in $\mathrm{C} / \mathrm{C}++$. There are two development options in such a case: implement the capabilities as Tcl commands, or implement the functionality in a separate process. Even if the latter option is chosen, it is desirable to build $\mathrm{Tcl} / \mathrm{Tk}$ interfaces to control such applications. Further, there are utilities and applications which use standard input and output to receive textual commands and retum results. Tcl/Tk interfaces to such processes prove useful as well.

The second alternative for such interfaces uses coprocesses, two cooperating processes in a master-slave relationship. The slave process reads commands from standard input, performs required operations (e.g., drawing graphics to an $X$ window) and returns information to standard output. The master directs the slave by writing its input and reading its output.

In this section we present a simple example of a Tcl/Tk master process and a slave written in $\mathrm{C}_{++}$and describe the technique of embedding windows. Source listings are given in Appendix F.

\subsection{SLAVE PROCESS}

By design, slave processes read commands from standard input. Clearly, the command language must be developed to meet the needs of the master and slave. Our example slave reads an $X$ Window dump file, permutes the points, and renders a specified number of points from the permuted image. Each new line of standard input is parsed as a command, in this case merely the number of points to display and a scale factor.

Slaves controlling windows must also respond to $X$ events. Figure 6-1 shows a portion of permute_view. cc which handles both input sources. A call to select () with read mask bits set for standard input $(0)$ and the $X$ display connection file descriptor blocks until either becomes active on input. $\mathrm{X}$ events are given priority.

\subsection{MASTER SCRIPT}

The Tcl/Tk script is the master and spawns the slave with a pipe for communication between them. Figure 6-2 shows the script fragments for spawning the slave process. Tcl opens pipes for standard input, output, and error. Thereafter, input from the file object reads from the slave's output, and output to the file object writes to the slave's input. Note that information flows in both directions. Figure 6-3 shows the writevalue procedure which outputs to the slave. The flush command is necessary in order for the slave to receive the output immediately. 


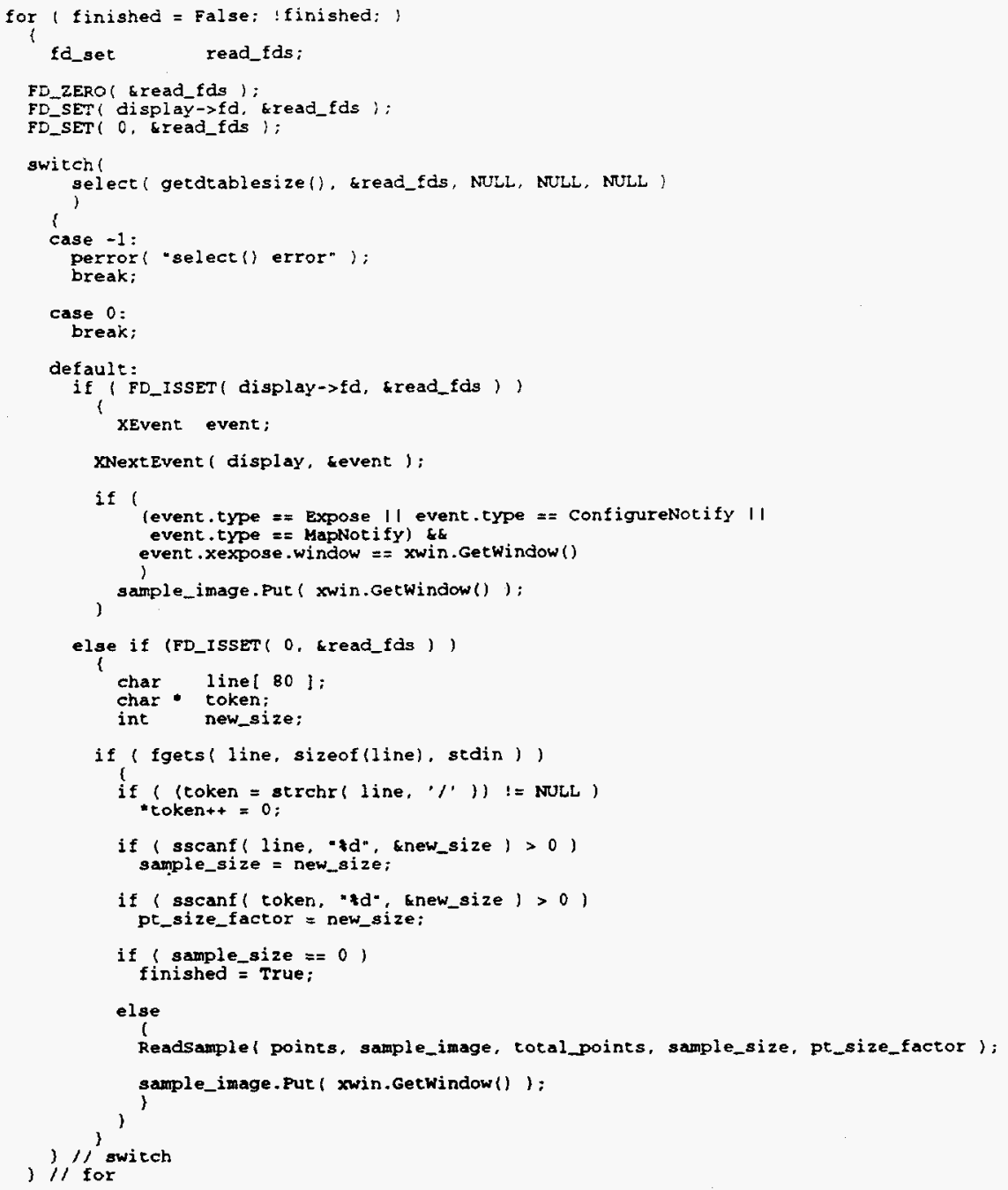

\section{Figure 6-1 Handling $X$ Events and Standard Input}

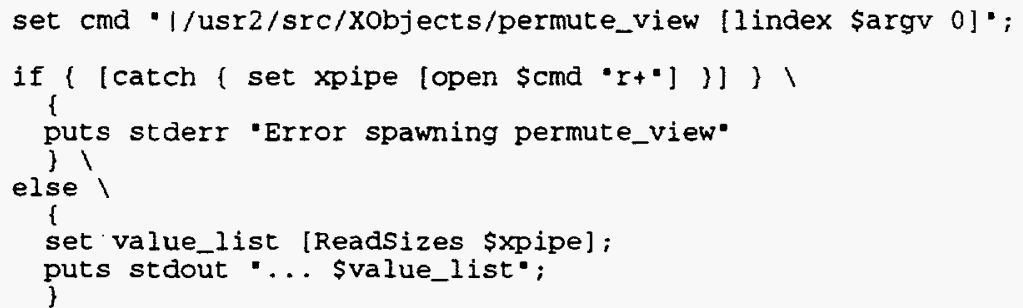

Figure 6-2 Spawning the Slave from Tcl 


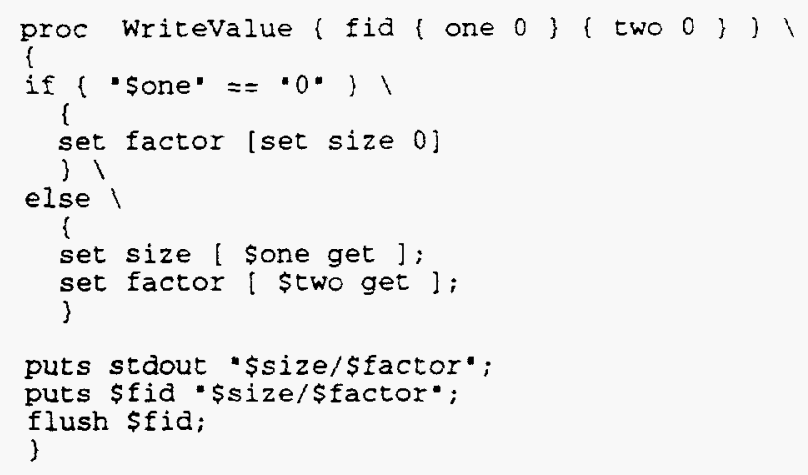

\section{Figure 6-3 Writing Output to the Slave}

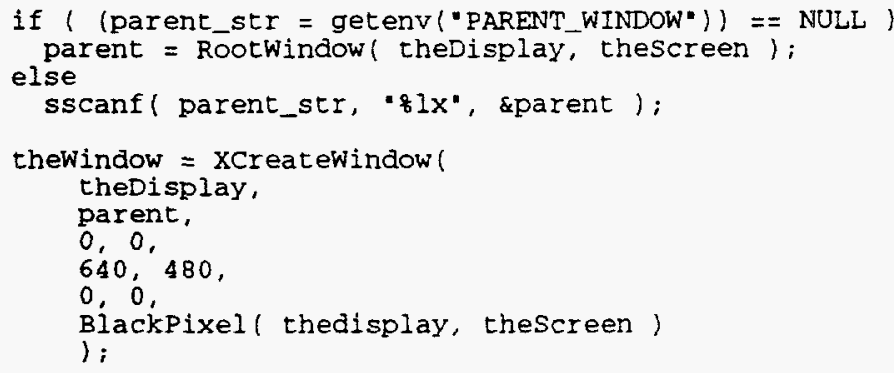

Figure 6-4 Slave Process Checking for Parent Window Handle

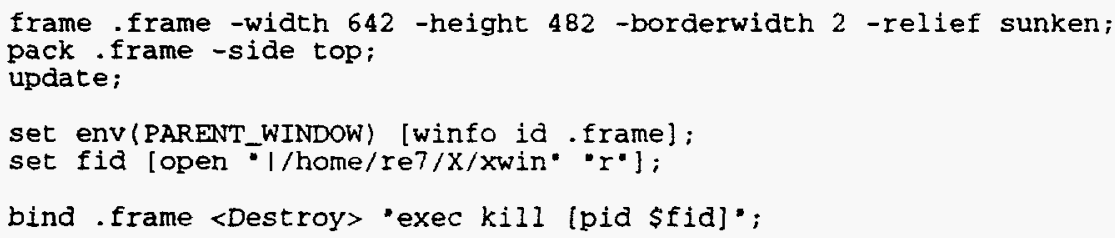

Figure 6-5 Tcl/Tk Commands to Embed Slave Window 


\subsection{EMBEDDING WINDOWS}

The permute_view slave creates its own window as a child of the root window of the $X$ display. Thus, the TclTk window and slave process window are separate on the screen. One may embed the slave window within a TclThk window very simply. Tk provides the winfo command to provide information on the $X$ windows it controls. The id subcommand returns a string with the hexadecimal value of a window's handle. This can be passed to an $\mathrm{X}$ window slave to be used as the parent parameter when creating a window. Figure 6-4 lists a code fragment of a slave process which checks the environment for the value of parent window handle. Figure 6-5 gives sample Tcl/Tk commands to embed the slave window within a frame widget and ensure proper termination of the slave process when the widget is destroyed. 


\section{SUMMARY}

$\mathrm{Tcl} / \mathrm{Tk}$ is a powerful and effective development environment for building Motif ${ }^{\mathrm{TM}} \mathrm{X}$ Window interfaces. Prototype efforts are especially aided by the ease with which Tcl/Tk scripts can be generated.

Extensibility allows the developer to embed new functionality within the language. Developers may program in the scripting language, extend the Tk widget set and drawing constructs, wrap Tcl interfaces around $3 G L$ code, and build TClTK and $3 G L$ coprocesses. However, the scripting language is limited in its support of structured development, and its use should probably be limited to interface objects and constructs. C++'s inheritance feature is especially useful in building Tcl classes on top of existing classes. 


\section{REFERENCES}

1. Booch, G., Object Oriented Design With Applications, Benjamin/Cummings, California, 1991.

2. Booch, G., and Bryan D., Software Engineering With Ada, 3rd Edition, Benjamin/Cummings, California, 1994.

3. Cox, B.J., Object Oriented Programming: An Evolutionary Approach, Addison-Wesley, Massachusetts, 1987.

4. Lee, R.W., and R. Hume, Geoserver: "A Dynamic Geographic Presentation System," Proceedings of the 1992 ACM Symposium on Applied Computing, Vol. 3, pp. 1265-1273.

5. Nye A. (editor), Xlib Reference Manual, O'Reilly \& Associates, 1992.

6. Open Software Foundation, OSF/Motif ${ }^{\mathrm{TM}}$ Release 1.2 Style Guide, Prentice Hall, Englewood Cliffs, NJ, 1993.

7. Ousterhout, J.K., TCl and the Tk Toolkit, Addison-Wesley, 1993. 


\section{APPENDIX A. TCLTK SOURCE LISTINGS}

\section{A.1 BARWINDOW.TCL}
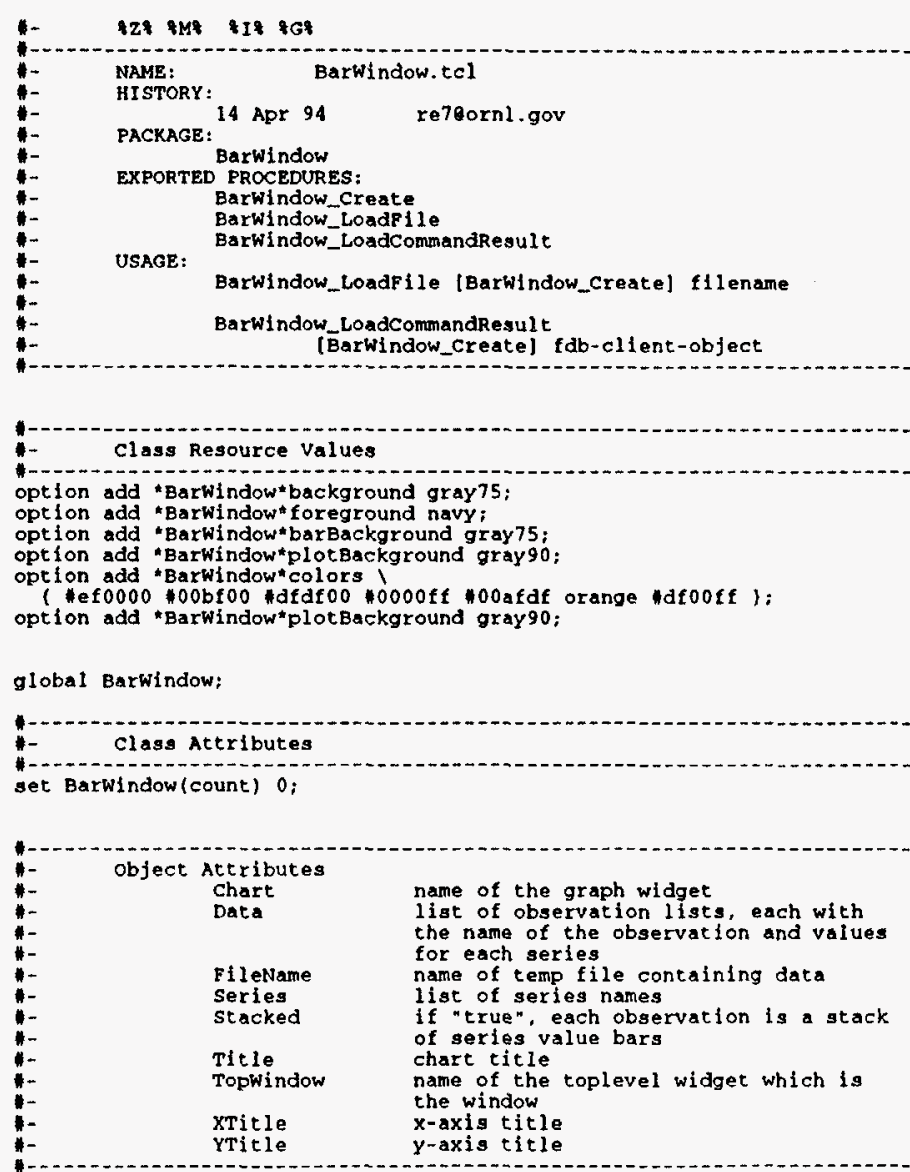

NAME:
HISTORY: 14 Apr $94 \quad$ re7eornindow_creat

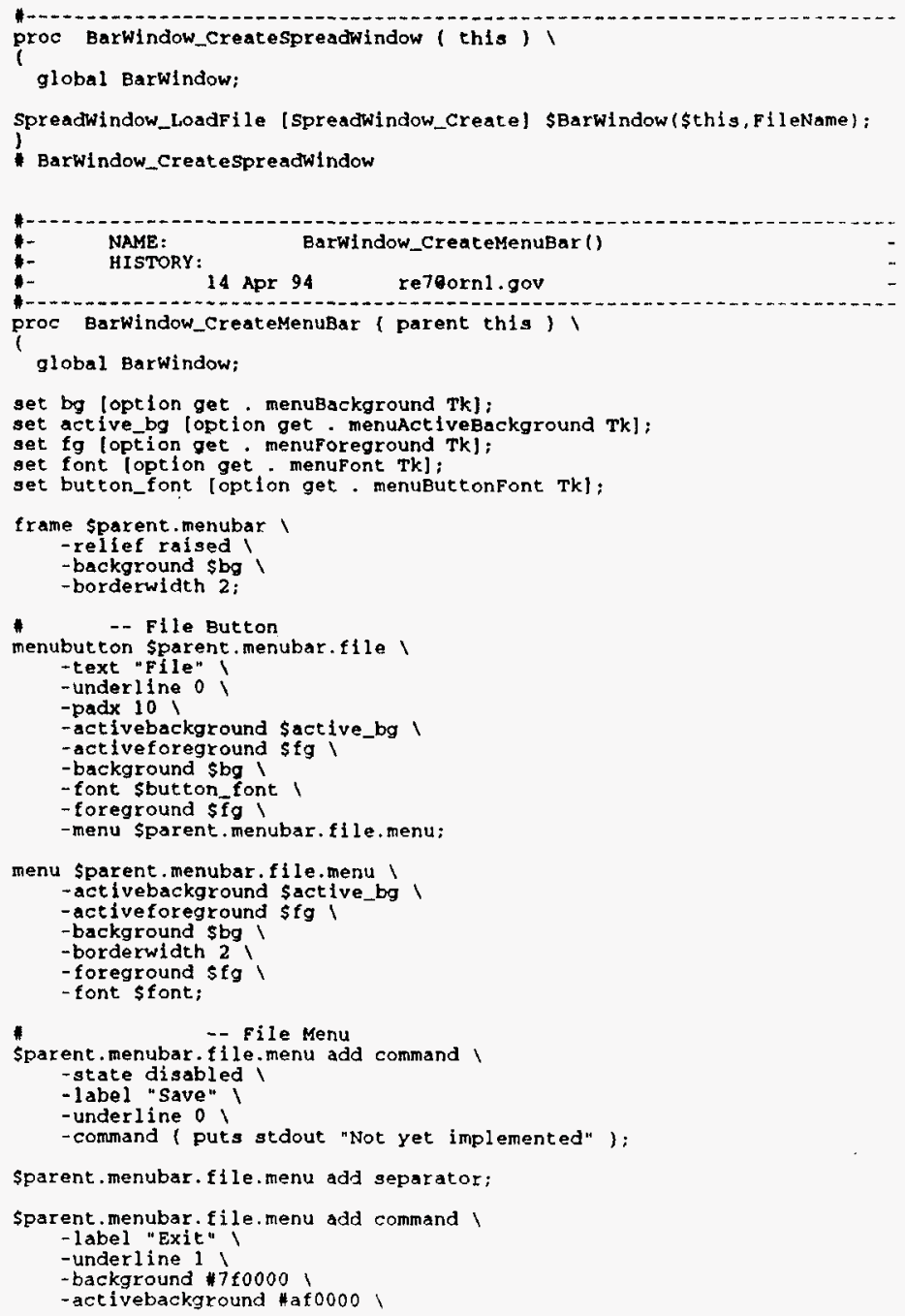


-command "destroy sparent"

menubutton Sparent. menubar.display 1 -text "Display"$$
\text { - -underline }
$$

-activebackground Sactive_bg I

- active foreground $\$ \mathrm{fg}$

- background \$bg - foreground $s \hat{f} g$ s -menu \$parent. menubar. displ ay. menu,

menu sparent menubar.display.menu - activebackground sactive . bg - activeforeground $\mathbf{s f g} \mid$

- background \$bg

- foreground $\$$

\$parent menubar. display.menu add command - label 1 Spread sheet "। - command "Barwindow_createspreadwindow sthis"

pack - - set up Menu

Sparent. menubar. file l
sparent.menubar.display - side left - 1 padx 5 :

bind sparent <Any-Focusin>

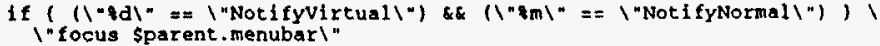

tK_menuBar sparent.menubar
\$parent.menubar.file;

return sparent.menubar

- Barwindow_CreateMenuBar

NAME: ${ }_{\text {HISTORY: }}{ }_{14}$ BarWindow_CreateGraph()
PURPOSE: ${ }_{\text {Creates the graph widget }}$

proc Barwindow_creategraph ( this ) ,

global Barwindow:

set top \$Barwindow(\$this, Topwindow):

blt_barchart schart !

- background loption get \$top background $\mathrm{TK}$ !

-plotbackground loption get stop plotBackground TK]

schart legend configure -background [option get \$top plotBackground Tk];

return schart:
" Barwindow_Creategraph

HAME:
HISTORY: 14 Apr $94 \quad$ BarWindow_Create()
PURPOSE: ${ }_{\text {Creates a Barwindow object }}$

proc Barwindow_create () ।

global Barwindow;

- - - set Name of This object

set this barwinsBarwindow (count)

- -- Name of This window

set name.

- - Buila Toplevel

toplevel Sname -elass Barwindow;

wm iconbitmap \$name option get \$name 1tbrary Barwindow]/barchart. xbm

wit lconmask sname

win title \$name "BarChart window - SBarwindow(count)"

- -- Bulla Frames

Barwindow_CreatemenuBar sname sthis:

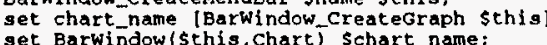

- -- pack Prames

pack \$name.menubar -side top-fill $x$ :
pack schart_name-side top -expand yes - $f 111$ both:

- -. Increment window count

incr Barwindow (count).

return sthis:

* Barwindow_create

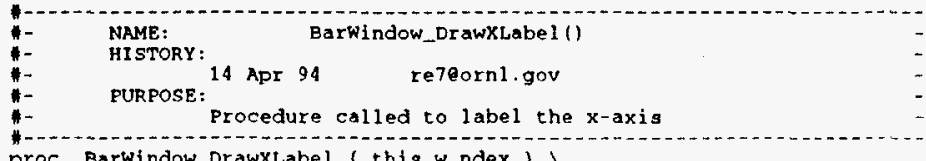

Barwindow DrawXLabel (this w ndex )

global Barwindow;

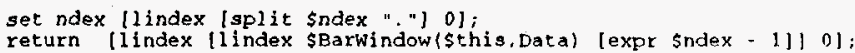

Barwindow_DrawXLabel

NAME:
HISTORY: 


\section{- PURPOSE: ${ }^{14}$ APY 94 re70ornl.gov

$$
\begin{aligned}
& \text { Builds the observation-series bars } \\
& \text { creates the graph widget }
\end{aligned}
$$

proc Barwindow_Bulldelements ( this )

global Barwindow;

set Barwindow(Sthis,Series) (split \$Barwindow(\$this, Series) $\because: "$ );

set top \$BarWindow (\$this, Topwindow);
set chart \$Barwindow (\$this Chart);

Schart xaxis configure !

-command "Barwindow_DrawXLabel \$this" ।

-font $9 \times 15$ ।
-title \$Bawindow (\$this, XTitle);

Schart yaxis configure ।

- ticle \$sarwindow(Sthis, yTitle):

schart conflgure I
-title \$Barwindow (\$this, Title),

Let sindex Buila Dummy Legend Elements

set sindex 0 ;
foreach ser \$Barwindow (Sthis, series) ।

schart element create dumny_s(ser) ।

-foreground [1index loption get stop colors Tk] ssindex] । incr ${ }^{-1}$ abel sser: incr

set bg loption get stop barBackground Tkl;

set oindex oy

dow (Sthis, Data) ।

Schart el ement create el_\$(oindex) ।

-xdata lexpr solndex +11 ।

- stacked SBarwindow (\$this, stacked) ।

-relief raised I

- fgl loption get stop colors Tk]

incr oindex;

Barwindow_ButldElements

NAME:
HISTORY; ${ }_{14}$ Aaxwindow_Parseattrs() $94 \quad$ refoornl.gov
PURPOSE: ${ }_{\text {Parse the attribute line in the data stream }}$

proc Barwindow_Parseattrs ( this line) I

global Barwindow;

foreach piece [split \$line "\t"] ।

set parts (split \$piece " $="]$;
set Barwindow(Sthis. (lindex sparts 0)) (1index sparts 1);
" Barwindow_Parseattrs

MAME:
HISTORY 14 Apr $94 \quad$ re7eornl.gov
PURPOSE:
Populate the data for the chart from the data flle

- proc global Barwindow,

- $\quad$-- Initialize Actributes

set Barwindow(Sthis, Title) "Could not open file sfilename",

set Barwindow (\$this, XTitle) "",

set Barwindow (Sthis, stacked) "true"

set BarWindow (Sthis, Data)

Bawindowisthis, FileName) "."

- -- open file

if ( leatch ( set fid lopen sfilename "r" $)$ ) = = 0) ।

set Barwindow(sthis, FileName) sfilename:

: -- Read Attribute tine

gets Sfid line;
Barwindow_parseaters sthis sline

set Barwindow(Sthis, Data) ();$$
\text { ! }
$$

while ( (gets sfld line) > -1)

lappend BarWindow(Sthis, Data) (split sline "It"l)

)

Barwindow_Bulldelements sthis
update:

- Barwindow_Loadfile

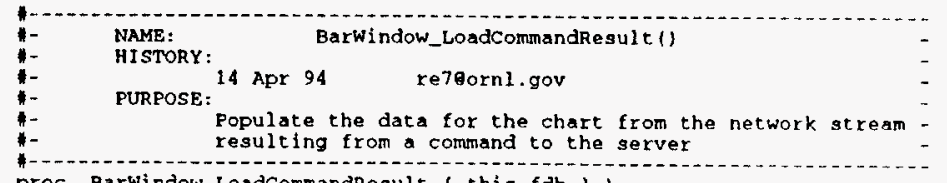

proc BarWindow_LoadCommandResult $(\mathrm{this} \mathrm{fdb}$ ) ।

global Fab Earwindow;

- -- Set Data file Name

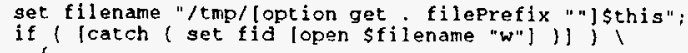

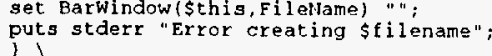

else । 
set Barwindow(\$this, Filename) sfilename;

- $\quad$-- Initialize Attributes

set Barwindow(Sthis, Title) "Error reading results";

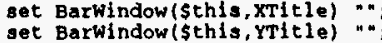

set Barwindow (sthis, stacked) "true";

set Barwindow (sthis, serles)

- I. Read Attribute Line

if ( "Istab ReadNextline line]" I = "eof" )

catch (puts \$fid \$line );
Barwindow_Parseattrs sthis \$line;

set Barwindow (\$this, Data) () ;

. -- Read Observation Lines

while ( "Isfdb ReadvextLine 1ine]" I= "eof")

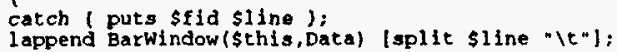
)

catch ( close Sfld):

BaxWindow_Buildelements Sthis:

update;

- Barwindow_LoadCommandResult

\section{A.2 FDB.TCL}

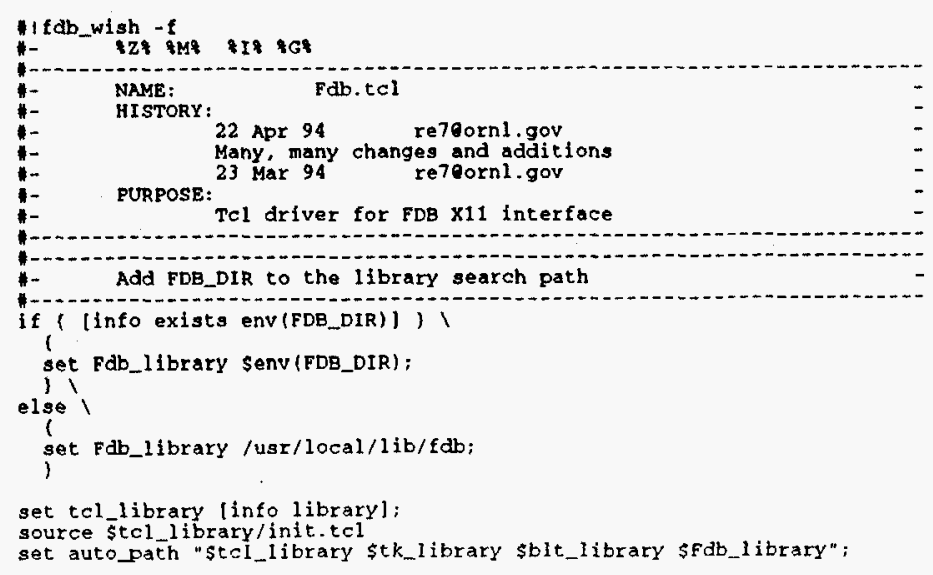

set tcl_precision 10;

\begin{tabular}{|c|c|c|c|c|}
\hline & set & Resources For Imported & cartes & \\
\hline $\begin{array}{l}\text { ption } \\
\text { ption } \\
\text { ption }\end{array}$ & $\begin{array}{l}\text { add } \\
\text { add } \\
\text { add }\end{array}$ & 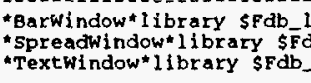 & $\begin{array}{l}\text { ary } \\
\text { ibrary: } \\
\text { orary: }\end{array}$ & \\
\hline & set & Resources & & \\
\hline $\begin{array}{l}\text { option } \\
\text { option } \\
\text { option } \\
\text { option } \\
\text { option } \\
\text { option } \\
\text { option } \\
\text { option } \\
\text { option } \\
\text { option } \\
\text { option } \\
\text { option } \\
\text { option } \\
\text { option }\end{array}$ & $\begin{array}{l}\text { add } \\
\text { add } \\
\text { add } \\
\text { add } \\
\text { add } \\
\text { add } \\
\text { add } \\
\text { add } \\
\text { add } \\
\text { add } \\
\text { add } \\
\text { add } \\
\text { add } \\
\text { add }\end{array}$ & 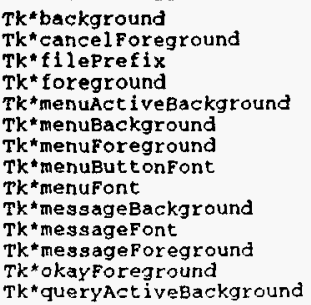 & 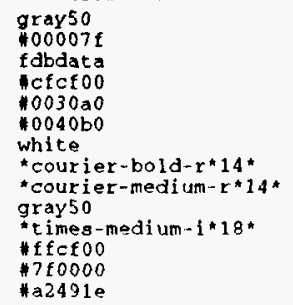 & $\begin{array}{l}\text { startupfile } \\
\text { startupFile } \\
\text { startupFile } \\
\text { startupile } \\
\text { startupfile } \\
\text { startupile } \\
\text { startupile } \\
\text { startupFile } \\
\text { startupile } \\
\text { startupFile } \\
\text { startupFile } \\
\text { startupile } \\
\text { startupFile } \\
\text { startupfile }\end{array}$ \\
\hline
\end{tabular}




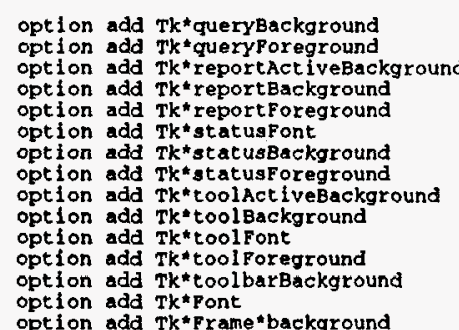

b2591e

yellow
000600

Hoosf00

thelvetica-medtum- $r+14 \cdot$ DarkGreen

yellow

gray65

courier-bold-r"14" 10000af

gray50 graurier-bold-r*14*

startupflle; startupfile: tartupFile; startupFile: startupFile:
startupfile: startupFile: startupFile startupFile tartupfile: startuprile: tartupFile;

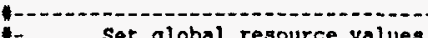
global Fdb:

set Fdb (menuBar) menubar;

set Fdb (megsage) Tmessage;

set Fdb (statusBar) .statusbar;

set Fdb(Queries) ।

( Budget ( "Cost Center Account" ) "Cost center Budget* spread ),

set Fdb(Reports)

( Test ("First parameter" "Second Parameter") -pard (CCSummary ( "Cost Center Account Substring"

( cCDumny () "Current Assumptions Example" text)

set Fab (Tools)

( budget.xbm "FindQuery Ccsummary" "“" )

DAME: DOEXIt proc DoExit i) i

global Fdb Fdb_library:

catch ( exec \$Fdb_library/clean loption get . fllepreflx " ") h

exit;

proc FindQuery (qname )
proc

global Fab;

foreach $\times \$$ Fab (Queries)

if " "[1 index $\$ \times 0] "==$ "\$qname" \}

Docommand query [1 ist $\$ x$ ] break;

Findquery

NAME:
proc Popup ( mgg )
global Fdb;

toplevel - Top Lopel - window

wm title poptop "Dlalog";

essage poptop.mes

-relief sunken

-justify center

-text smsg:

- . Buttons

button poptop. button_frame .ok
-text "OK.

-text "OR."
-background [option get. toolsackground Tk]
-foreground [option get. OkayForeground TK]

- activeforeground foption get : oksyForeground Tk] I $\mathrm{Tk}$ । - font loption get . tool Font $T \dot{k}$ ]

pack

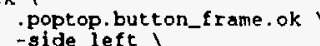

poptop. button_f rame.ok

- padx 20
- ipadx 2

,

.poptop.message I
.poptop.button_f rame

- side top

$$
\text { -pady } 5 \text {; }
$$

tKwait visibility Dialog window come up

grab poptop;

* popup

NAME: Rundialog()
proc RunDialog (dlist i

global Fdb dialog_values run_flag

toplevel - Top Level window

-background loption get toolBackground Tkl

wm title top "Parameter Dialog"; 


\section{Writestatus "Sending command to FDB server ..." ;}

if ( [\$Pab(fdbclient) Getstatus $]$ 1=0)

Writestatus "Send failed 11";

else'

Writestatus "Reading query results ..."

set wtype (11ndex sqlise 3):

If ( "\$wtype" == "spread" ) ।

spreadwindow_LoadcommandResult [Spreadwindow_create] $\$ F d b$ (fdbclient)

elseif ( "Swtype" == "bar") ,

Barwindow_LoadCommandResult (Barwindow_Create) \$Fab(fabclient) else'

TextWindow_LoadCommandresult (TextWindow_Create) SFdb(fdbclient)

writestatus "Query complete":

Docommand

NAME: CreateMenuBar ()
proc createMenuBar (\}$)$

global Fdb;

set bg loption get. menuBackground $\mathrm{Tkl}$,

set $f g$ loption get . menuforeground $T k$ l ;

set font loption get menuFont TKl; ;
set button font loption get. menuButtonfont Tkl:

\section{frame menubar 1}

-relief raised
-background $\$ b g$

-borderwideh 2;

session button

- underline o!

-padx 101

ont \$button_fone

-activebackground sactive_bg

- $f$ oreground $\$ \mathbf{f g}$

- act lve foreground sfg !

menu -menubar.session.menu 1

-activebackground \$active_bg

- background \$bg 1

-borderwidth 2 ।

foground sig

- Menubar session menu a - session menu

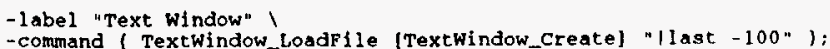
.menubar. session. menu add command
-1 abel "Message of the Day"

global rab;

writestatus "Sending command to FDB server ..."

"\$Fab(fabclient) sendCommand "cat /etc/motd";

Writestatus "Reading servez response ..." "; writestatus "Dialog complete":

menubar.session.menu add separator;

menubar.session.menu add command -label "Exit" -background 7 f 0000 - activebackground "af 0000

- Qnubutton - Queries butto -text "Queries

- padx 10 ।

- font sbutton_font

-activebackground sactive_bg । - activeforeground $\$ \mathrm{fg} !$
- menu . menubar.quextes.menu;

menu menubar.queries. menu

-borderwidth 2

-background loption get . queryBackground Tk]

- foreground loption get queforeground [opt ion get queryForeground $\mathrm{Tk}$ ].

foreach rep \$Fdb(Queries) । Queries menu

menubar. queries menu add command

-label [1index srep 2] activebackground [option get querYActiveBackground $T k$ ]

-- Reports button

ubutton .menubar.reports 1

- tunderline 0 .

- font sbutton_font

-background $\$$ bg

- activebackground sactive_.bg

- foreground $\$ \mathrm{fg}$ - activeforeground $\mathrm{sfg}$

- menu . menubar, reports. menu

menu .menubar reports.menu

- font sfont 2

-background loption get reportbackground $T k$ l ; 
-activeforeground loption get . reportForeground $\mathrm{Tk}$ ];

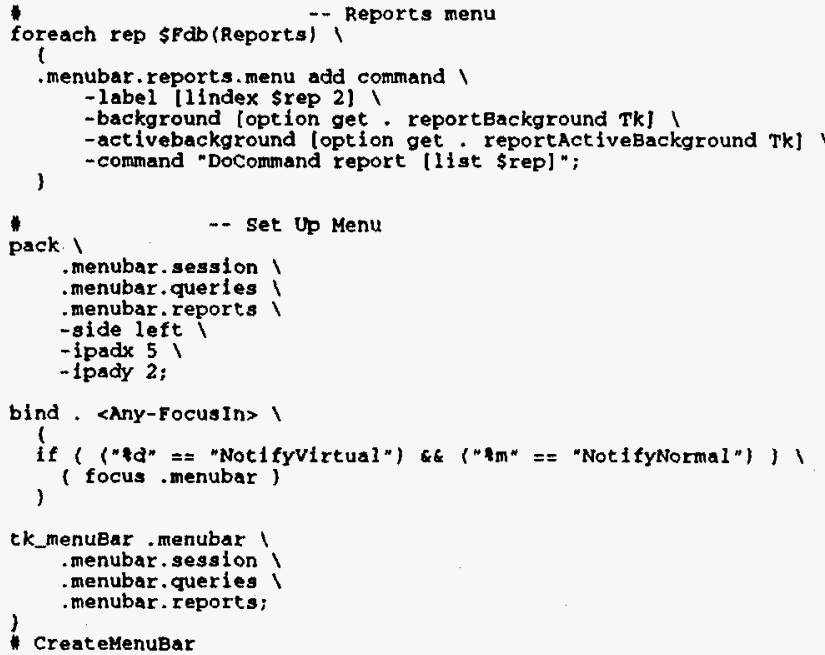

bind . <Any-rocusin>

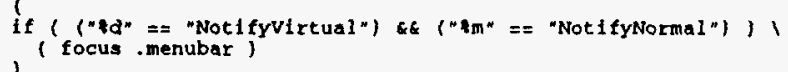

tk_menuBar - menubar I menubar. session -menubar. queries

- createmenuBar

CreateToolBar(1
proc CreateToolBar 01

global Fab Fab_library;

frame toolbar

background loption get . toolbarBackground $T K$ l

set count of
foreach tool sFab (Tools)

button toolbar.toolscount

-bitmap osfdh library/litindex stool of

activebackground loption get. toolActiveBackground $T k_{1}$ ।

-activeforeground lopt lon get. toolforeground $\mathrm{Tk}$ ) ।

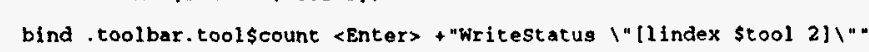

pack toolbar. toolscount I

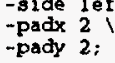

incr count;

createToolBar

NAME:

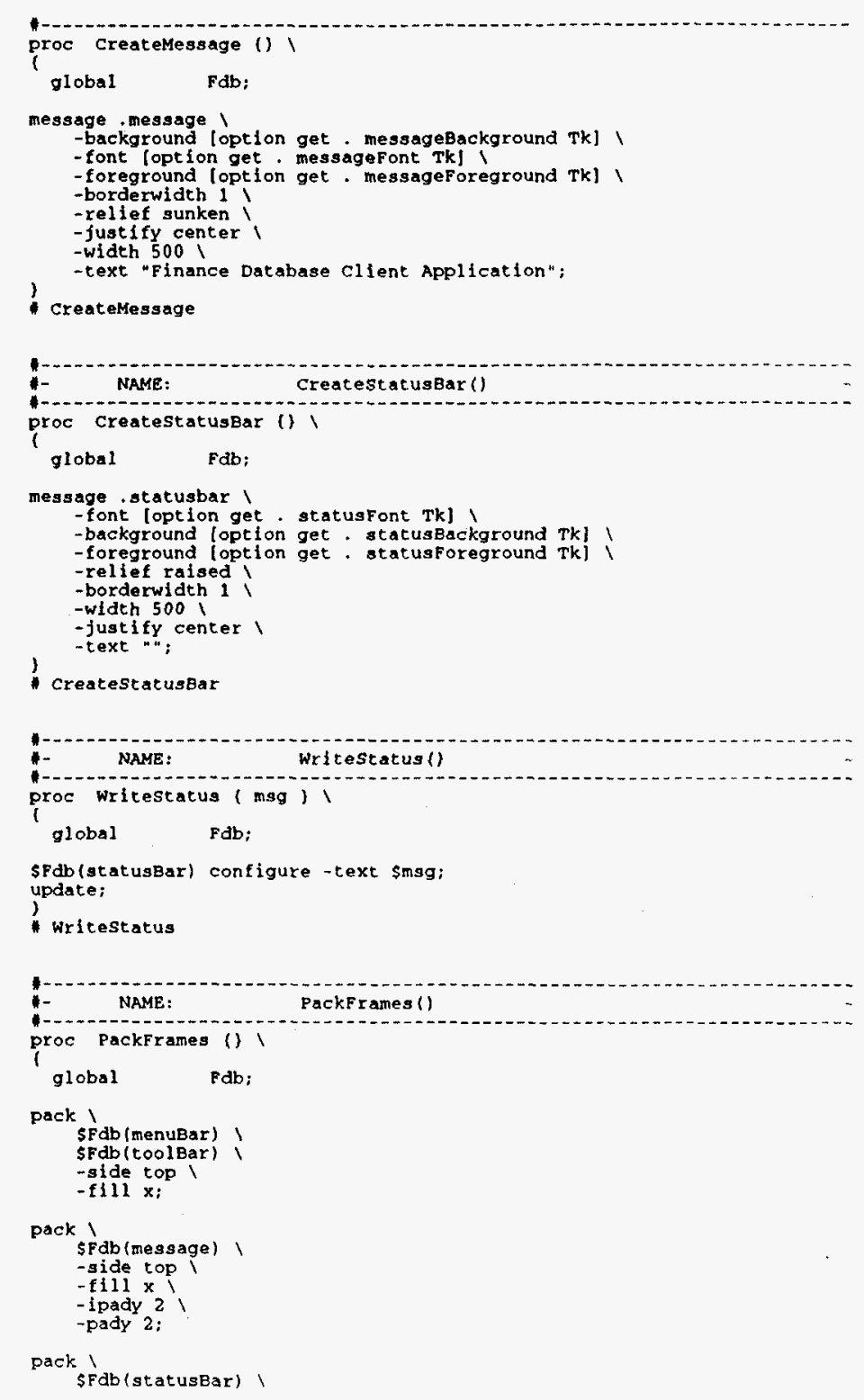




\section{-side top \\ -pady 2; \\ ? PackFrames}

mains

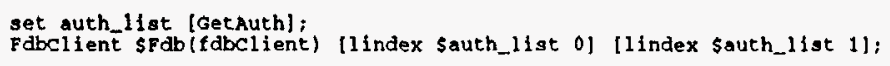

if ( "(fdb Gerstatus)" i= "0" ) ,

Popup "User Authentication Palled";

$\sum_{\text {else }}^{\text {exit; }}$

thm t1C16. "FDE VaR8.7LR";

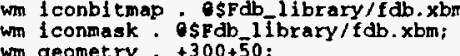

Createmenuiar;
CreatereroolBar;

Createmessage:
Creacesstatusair

writestatus "User Authentication successful";

in deleonify .:

\section{A.3 SPREADSHEET.TCL}

NAME:
HISTORY:

global spreadsheet;

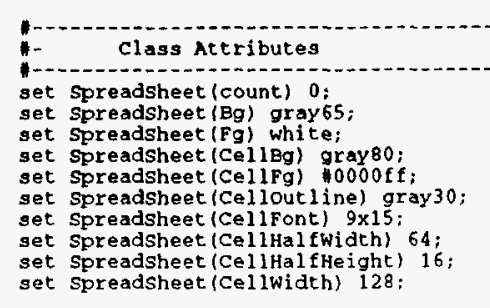

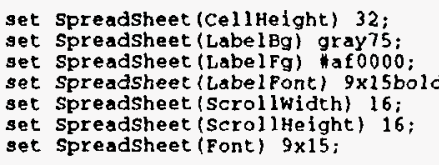
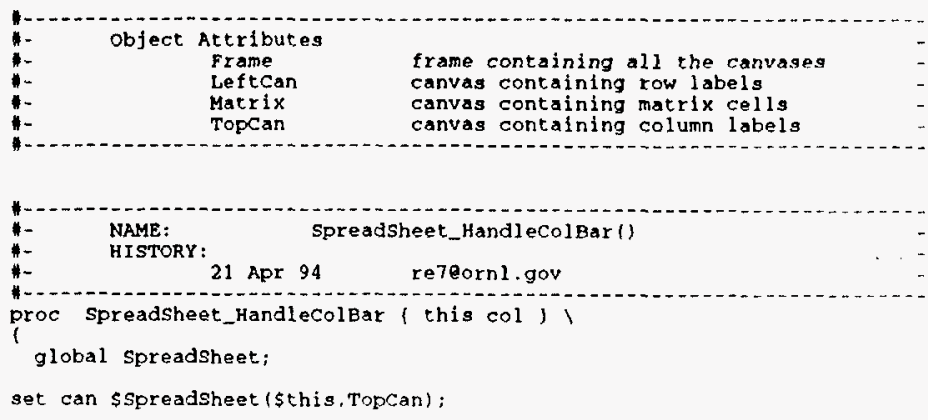
set matrix \$Spreadsheet (\$this, Matrix) :

1f ( "[1index [\$can itemconfigure colbar_\$col -relief] 4]" == "raised" ) Scan itemconfigure colbars -rellef raised;
\$can itemconfigure colbar_scol -relief sunken

- Smatrix itemconfigure cell_col_\$col -outline \$Spreadsheet(Celloutline) else 1

$\$$ can temeonfigure colbar_\$col -rellef raised;

Spreadsheet_Handl eColBar

NAME:
HISTORY: ${ }_{21}$ Apr $94 \quad$ Spreadsheet_HandleRowBar 11
-2 re70ornl.gov

proe spreadsheet_HandleRowBar ( this row)

global spreadsheet;

set can \$spreadsheet (\$this, LeftCan);
set matrix \$spreadsheet ( $\$$ this, Matrix)

If $\{$ "[lindex (\$can ftemconfigure rowbar_\$row -relief] 4 ]" == "raised") Scan itemconfigure rowbars -relief raised;
Scan itemconfigure rowbar $\$$ row -relief sunken

- \$matrix itemconfigure cell_row_\$row -out 1ine \$spreadsheet(Celloutline), else !

scan itemconfigure rowbar_srow -relief ralsed

Ispreadsheet_Handl eRowBar

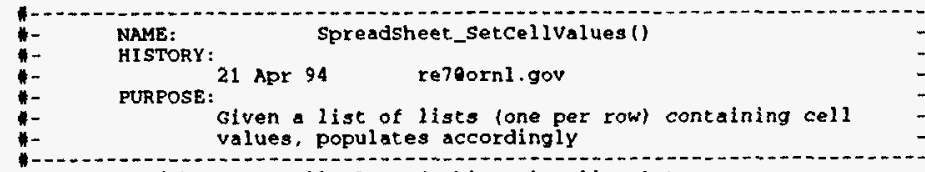

proc spreadsheet_setcellvalues ( this value_list) i

global spreadsheet:

SpreadSheet (\$this, LeftCan) delete celltexts;

set rindex

(c)

foreach row_list \$value_list

sat eindex 0 foreach value srow_list

\$spreadsheet(Sthis. Matrix) create text

\$tags "celltexts celltext_s(rindex)_s(cindex) " ।

- anchor o '

-fill \$spreadsheet (Ce11 rg)
Incr xpos Sspreadsheet (Cellwidth),

incr clndex

Incr ypos sspreadsheet (CellHeight);

I spreadsheet_setcellvalues

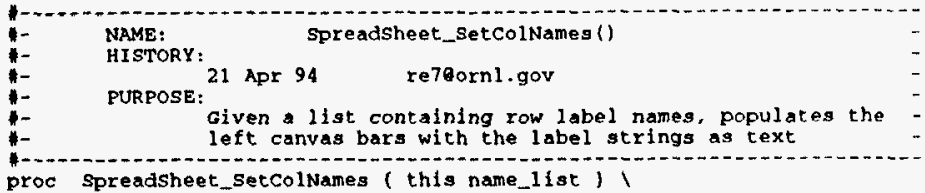

Droc soreadsheetsercolvames ( this name list) 1

global spreadsheet;

sspreadsheet (sthis, Leftcan) delete coltexts;

set cindex 0 ;

\$spreadsheet (\$this, Topcan) create text

Sxpos \$Spreadsheet (Cell half height)

- anchor c l
-font SSpreadsheet (Label Font)

- text Sname '

\$spreadsheet (\$this, Topcan) bind coltext $\$ c$ index <But onkelease-1>

incr cindex;

NAME:
HISTORY: 21 Apr 94 SpreadSheet_SetrowNames 0
PURPOSE:
Given a list containing row label names, populates the
left canvas bars with the label strings as text

proc spreadsheet_SetRownames ( this name_list)

global Spreadsheet:

* -- Delete Existing Text Items

\$spreadsheer (\$this, Leftcan) delete rowtexts

set rindex 0;

set ypos S.SpreadSheet (Cell Hal fHeight)

oreach name sname_list

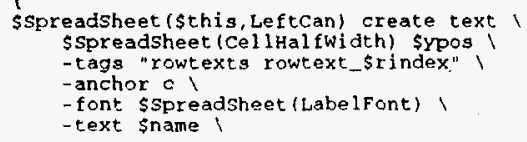


- fill \$Spreadsheet (Label Fg);

\$spreadsheet (\$this, Leftcan) bind rowtext_\$rindex <ButtonRelease-1>

Incr rindex;
Incr ypos $\$$ Spreadsheet (Cell Helght);

? spreadSheet_setrownames

NAME:
HISTOR: 21 Apr $94 \quad$ re7eornl.gov
FURPOSE:
Given a list contalning row values (label followed by
column values), populates the spreadsheet

proc spreadsheet_Setrowvalues ( this rv_list)

global spreadsheet;

- -- Delete Existing Text Items

Sspreadsheet (sth1s, Leftcan) delete rowtexts;

- -- Process Each List Item as a Row

set ypos \$Spreadsheet (Cell Hal fHeight):

foreach row_list Srv_list

- -- create Label rext

sspreadsheet (\$this, Leftcan) ereate text spreasheet (cellHal fwidth) \$Ypos । - anchor $c 1$

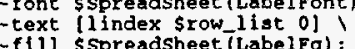

Spreadsheet (\$this, Leftcan) bind rowtext_\$rindex <ButtonRelease-1>

:

set cindex 0 ;
set $x$ xpos $\$$ Spreadsheet (Cel l Hal fWidth); -- Process Remaining Items in sublist as column values

foreach value (1range \$row_list 1 end) ।

sspreadsheet (sthis, Matrix) create text

\$xpos \$ypos 1 igs celltext_s(rindex)_s(cindex)"

- font sspreadsheet (Cellfont)

- text Svalue $\$$ Spreadsheet (CellFg)

incr xpos $\$ s p r e a d s h e e t$ (cellwidth)
incr cindex:

incr ypos sspreadsheet (Cell Height):

iner rindex;

Spreadsheet_Setrowvalues

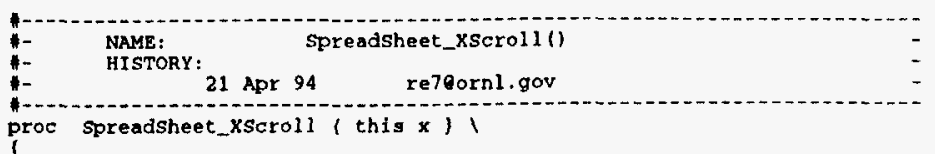

global spreadsheet:

\$spreadsheet (\$this, Matrix) xview $\$ x$;
\$spreadsheet ( $\$$ shis, Topcan) xvi ew Sx:

spreadsheet_xscroll

NAME:
HISTORY: 21 Apr $94 \quad$ SpreadSheet_YScroll1)
proc SpreadSheet_YScroll (th1s y )

global spreadsheet:

\$SpreadSheet (\$this, Matrix) yoiew \$y;
sspreadsheet ( $\$$ this, Lettcan) yview sy;

Spreadsheet_yscrol

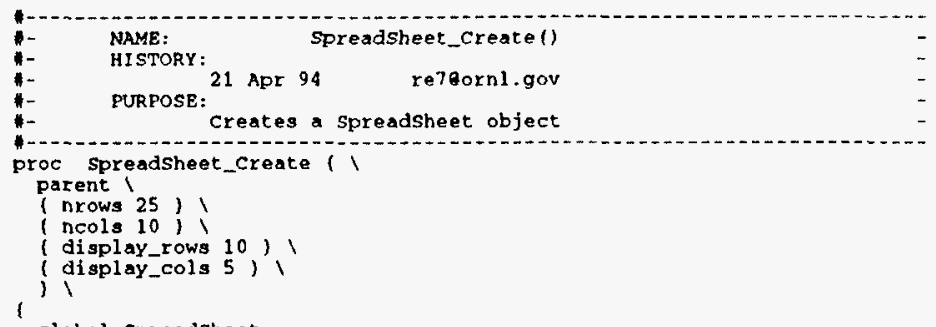

'global spreadsheet;

- - Get Name of New object

set name spreadsspreadsheet (count):

- $\quad$-- Compute Canvas display values

if (sdisplay_cols > 5ncols) ( set display_cols sncols )

If (sdisplay_rows > snrows ) ( set display_rows snrows

set display hathe (expr

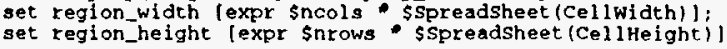

- - - container frame

frame Sparent. \$ (name)_frame
-background $\$$ Spreadsheet (Bg) relief sunken:

set Spreadsheet (sname, Frame) sparent. \$(name)_frame;

" $\quad$-. Packing Dummies 
frame sparent. S (name) frame. right -widh $\$$ spreadsheet (Scrollwidth)

pack sparent.S(name)_frame.right -side right -fill y;

frame Sparent. $\$$ (name)-frame. left I

-width \$spreadsheet (Celiwidth)

-borderwidth 1;

pack sparent. $\$$ (name)_frame.left -side left -fill $Y$ :

frame sparent.\$(name)_frame.right, bottom । - background \$SpreadSheet (Bg) '

pack sparent. \$ (name)_frame. right. botrom -side bot tom;

frame sparent. 5 (name) f frame.right. top 1

background SSpreadsheet (Bg) I

pack \$parent. \$(name)_frame, right. top -side top

frame sparent. $\$$ (name)_frame. left.bottom background \$Spreadsheet (Bg)
height \$SpreadSheet (Scroll Height)

pack sparent $s$ (name) frame left.bottom -side bottom

frame \$parent. $\$$ (name) frame. left. top
-background $\$$ Spreadsheet (Bg) background \$Spreadsheet (Bg)
-height \$Spreadsheet (Cell Height)

-borderwidth $1 ;$
sparent. $\$$ (name)_frame. left.top -side top;

- -- Top Canvas

canvas Sparent. $\$$ (name) frame.topcan 1

h 'spreadsheet (CellHeight)" background sspreadsheet (Bg)

-borderwidth 1

pack Sparent. \$(name)_frame. topcan $-s i d e$ top -fill $x ;$
set spreadsheet (\$name, Topcan) Sparent. \$(name)_frame.topcan

- -- Left Canvas

canvas sparent. $\$$ (name) frame. left. leftcan

width \$Spreat

readsheet (Cellwidth) sregion_height " -background \$Spreadsheet (Bg)

-scrollincrement sspreadsheet (Cell Height):

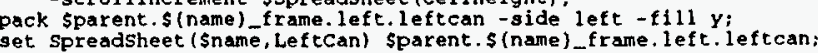

* -- Bottom Scrollbar

serollbar sparent.\$(name)_frame.xscroll

relief sunken

-width \$spreadsheet (ScrollHeight) ।

-command "SpreadSheet_xscroll Sname";
pack \$parent. \$(name)_frame. xscroll -side bottom -fill x

* - - Right Scrollbar

scrollbar Sparent.\$(name)_f rame.right.yscroll -orient ver 1
-width \$Spreadsheet (scrollwidth) ।

pack Sparent. \$(name)_frame.right.yscroll" -side right -fill y,
-command "Spreadshet_YScroll \$name";

- -- Matrix Canvas

anvas Sparent. $\$$ (name)_frame . matrix

height sdisplay helight

scrollregion "0 0 \$region_width \$region_height" ।

-background spreadsheet(bg)

(cellHeight) ।

- xscroll "Sparent. S (name)-frame. xscroll set" ' '

pack sparent. S(name) frame-matrix-side top-expand yes -fill both

frame.matrix:

- - Create Buttons and Cells

set row $0 ;$ set ypos 0 ) 1

( srow < snrows) ' 1 incr row; incr ypos sspreadsheet (Cell Height),

('parent s(name) frame.left.leftcan create bar

sspreadsheet (Cellwidth) [expr \$ypos + sspreadsheet (Cell Height)] ।

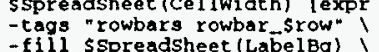

Sparent.S(name)_frame.left. leftcan bind rowbar_srow <ButtonRelease-1>

for

(set col $0 ;$ set xpos 0 ) ।

(incr col; incr xpos sspreadsheet (Cellwidth) \} ।

if ( srow $=0$ ) )

sparent. $\$$ (name)_frame. topcan create bar

5xpos 0 lexpr 5 xpos + sspreadsheet (cellwidth)| sspreadsheet (CellHeight) -tags "colbars colbar scol"

- fill \$Spr

Sparent. \$(name)_frame, topcan bind colbar_\$col <ButtonRelease-1>

sparent.\$(name) frame.matrix create rectangle ।

(expr \$xpos + \$Spreadsheer (Cellwidth) - 1 1

- tags "cells cell_row stow cell_col_scol cell s(row) s(coll)"

- out line sspreadsheet (Cellout I ine) ।

return Sname;

Spreadsheet_create 


\section{A.4 SPREADWINDOW.TCL}

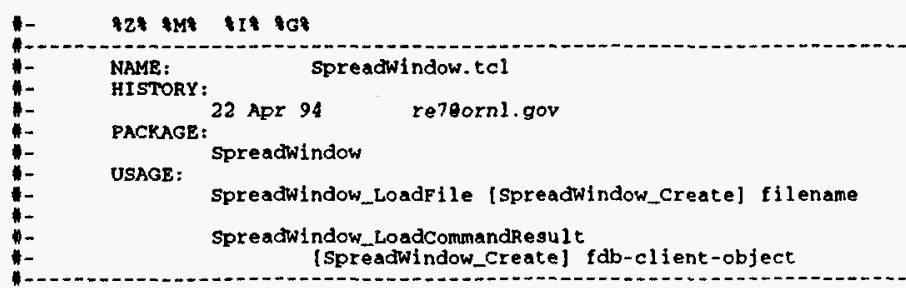

globa I spreadwindow:

Class Actributes

set Spreadwindow (Bg) gray 75 ;

set spreadWindow (TitleFg) navy;
set SpreadWindow (Titlefont) $10 \times 20$

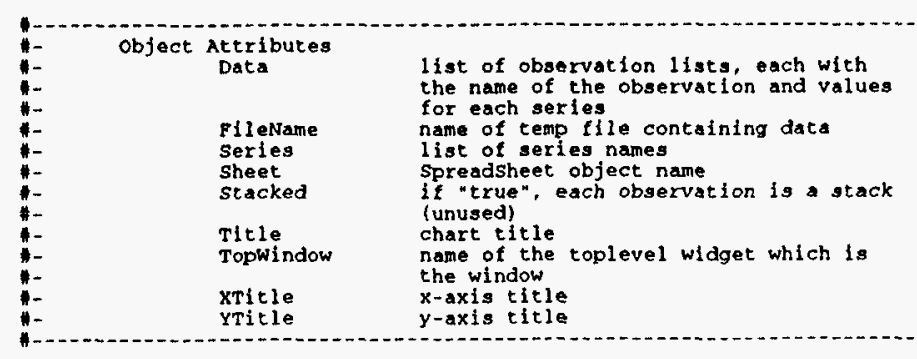
NAME: SpreadWindow_CreateBarwindow()
"- PURPOSE: 22 Apr 94 re7eorn1.goV
Creates a Earwidnow object from the data file

proc spreadwindow_createBarwindow ( this ) ।

global Spreadwindow:

Barwindow_LoadFlle (Barwindow_Create) sspreadwindow (\$this, FileName);

Spreadwindow_CreateBarwindow

NAME:
HISTORY:

proc spreadwindow_createmenubar (parent this) i
' global Fdb spreadwindow;

set bg loption get menuBackground $T k$ l,

set fg loption get - menuforeground $\mathrm{Tk}$ ]

set font loption get . menuFont TK]:

frame sparent.menubar

-relief raised 1
-background $\$ \mathrm{bg}$ ।
-borderwidth 2 ;

menubution sile Button -text "File" !

-underline 0

padx 10 r 01

_activeforeground sactive_bg

-background shg 1

- font sbutton-font

- foreground $s$ fg $l$
-menu sparent, menubar. file.menu

menu sparent.menubar. file. menu 1 background \$bg ।

- foreground $s \mathrm{fg}$

foreground
font sfont;

s

arent menubar file, menu add command I - state disabied

- - undel "Save"

- command ( puts stdout "Not yet implemented" ,

sparent.menubar.file.menu add separator:

sparent menubar file menu add comend

- Iabel "Exit"
-underline 1
-

background 7 f 0000

-activebackground "af 0000
-command "destroy sparent."

- -- Display Button

menubar. di splay - text "Display"
- underline 0 .

- actix lo - activeforeground $\mathbf{s} \mathrm{fg}$

- background Sbg 1

- font Sbutton $\mathrm{fo}$

- foreground $\$ \bar{f} g$ ।

menu \$parent. menubar. di splay.menu

activeforeground $\$ \mathrm{fg}$ ।

-background $\$$ bg : 
-borderwidth 21

-foreground $\$$ f

Darent Display Menu

add command

-underline 1 '
-command "Spreadwindow_createBarwindow sthls"

sparent.menubar.display.menu add command ।

- label " Line Graph " I

- underline 1 ' command ( puts stdout "Not yet implemented" ,

-

$$
\text { -- set up Menu }
$$

sparent. menubar. file ।

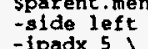

- ipadx 5 ;

bind sparent <Any-Focus In>

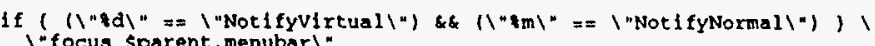

Ck_menuBar Sparent.menubar
Sparent.menubar.file;

return sparent.menubar

Spreadwindow_createmenuBar

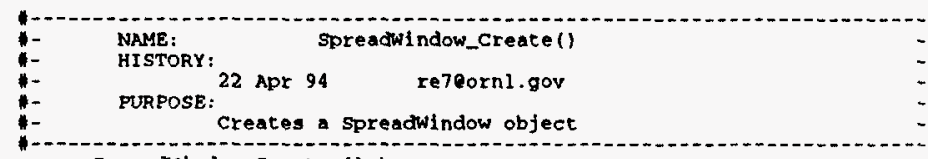

proc Spreadwindow_create (i)

global spreadwindow Fdb_llbrary:

- -- Name of This object

set this spreadwins Spreadwindow (count),

set name . swtop\$Spreadwindow (count);

-- Buila Toplevel

toplevel sname

wm Iconnask Sname esFab_library/spread. xbm

wm minsize $\$$ name $300200 ;$
wII eitle \$name "spreadsheet window - \$spreadwindow(count)"

Spreadwindow_createmenuBar sname sthis

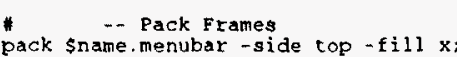

... Increment window count incr Spreadwindow (count);

return sthis:

- spreadwindow_create

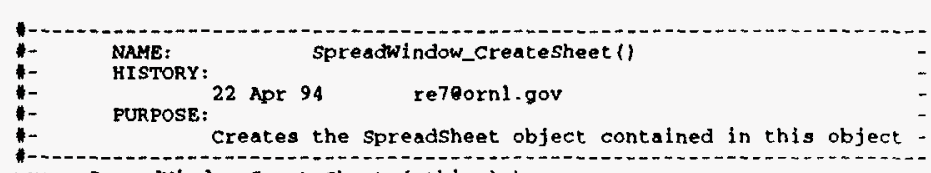

proc spreadwindow createsheet ( this) )

global spreadWindow spreadsheet;

- create mitte uabel

label sspreadwindow (\$this, Topwindow) . title -background \$Spreadwindow(Bg) -text sspreadwindow (Sthis, Title):

- -- create Spreadsheet widget

et sheet

ISpreadsheet_create I

11 length \$Spreadwindow (\$this, Data) I ।

set spreadwindow (\$this, Sheet) \$sheet;

Spreadsheet_SetcolNames \$sheet \$spreadwindow(Sthis,Series);

Spreadsheet_SetrowValues ssheet \$Spreadwindow(sthis, Data)

pack \$spreadwindow (5this, Topwindow). title ।

- side top 5

pack \$spreadsheer (\$sheet, Frame) I

- Spreadwindow_createsheet

NAE:
HISTORY: 22 ApreadWindow_Parseattrs() 94 refeornl.gov
PURPOSE:
parse the attribute line in the data stream

proc Spreadwindow_Parseattrs (this line)

global spreadwindow,

foreach piece [split sline "\t"| |

set parts lsplit spiece " $=1$ i

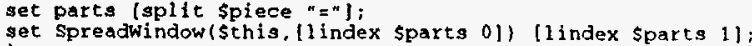

Spreadwindow_Parseatcrs

HAME: Spreadwindow_LoadFilei 


\section{HISTORY: ${ }^{22}$ Apr 94 re76ornl.gov
PURPOSE:
Populate the data for the chart from the data file}

proc spreadwindow_Loadfile (this filename)

global spreadwindow Fdb_library;

- -- Initialize Attributes

set spreadwindow (sthis, Tit1e) "Could not open file \$filename";

set spreadWindow (Sthis, XTitie) "

set spreadwindow (\$this, stacked) "true",

set spreadwindow (sthis, ser1es) " "

set Spreadwindow(Sthis, Data) (); "
set spreadwindow (Sthis, FileName) ",
set spreadwindow (Sthis, sheet) " ";

- -- open fille

if ( catch ( set fid lopen sfilename " $x$ "I $)==0$ ),

1 -- save file name

set Spreadwindow(\$this, Filename) Sfilename;

" -- Read Attribute Line

\section{gets Sfld line;}

Spreadwindow_ParseAttrs Sthis $\$ 1$ ine:

SpreadWindow (Sthis, Series)
(split sspreadwindow(Sth1s, serles) " : "l:

set spreadwindow(\$this, Data) ():

while 1 laets

while ( Igets s̈fia line $>-1\} \mid$

lappend spreadwindow(\$this, Data) (split sline "|t"l:

- -- create spreadsheet

Spreadwindow_createsheet sthis;

update;

Spreadwindow_Loadfile

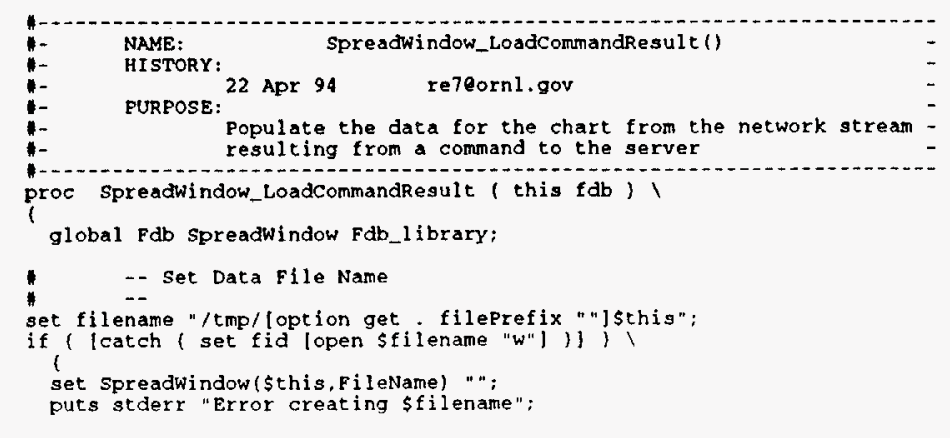

elise

set Spreadwindow(sthis, FileName) \$filename;

- -- Initialize Attributes

set SpreadWindow (\$this.Title) "Error reading results"

set spreadwindow (Sthis, xTitle) "" "

set spreadwindow (Sthis, Stacked) "true"

set Spreadiwindow (sthis, Series)

set spreadwindow (\$this, Data) ():

- - Read Attribute Line

if $($ "[sfäb Readvextline line $] " 1=$ "eof" 1

catch (puts sfid sline);

set Spreadwindow (Sthis, Series)

set spreadwindow (sthis, Data) ()

while ( "(\$fab ReadNextLine line)" $t=$ "eof")

catch (puts sfid sline)

lappend Spreadwindow (Sthis, Data) [split sline "It"l:

--- create spreadsheet

Spreadwindow_Createsheet sthis;

catch ( close sfid) :

update:

SpreadWindow_LoadCommandResult 


\section{A.5 TEXTWINDOW.TCL}

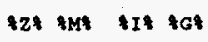

NAMB: Textwindow.tel

4 Mar 94 re78ornl.gov

PACKAGE:

USAGE: Textwindow

Textwindow Loadpile [Textwindow Create f filename

TextWindow_LoadCommandResult (a)

global Textwindow:

class Attributes

set Textwindow(Bg) gray30;

set Textwindow (Font) $9 \times 15 ;$
iset Textwindow (Font) $8 \times 13$

set Textwindow(count) 0

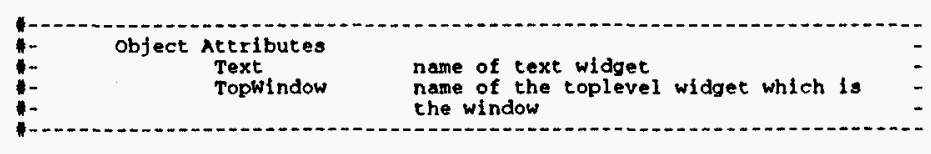

NAME:
HISTORY 4 Mar $94 \quad$ TextWindow_CreateMenuBar ()
reteornl.gov

proc rextwindow_CreatemenuBar ( parent this ) ,

global Fdb Textwindow;

set bg loption get menuBackground TkJ;
get active bg lopt ion get. menuActiveBackground TKl;

set fg loption get. menuForeground $T k$ l

set font loption get menuFont TK] ;
set button_font loption get. menuButtonfont $\mathrm{Tk}$ ]

frame sparent.menubar ।

background $\$ b g$

width 2

- -- File Button

button sparent.menubar. file

- text pile"

activebackground sactive_bg

activeforeground \$fg

- fackground sbg

- menu \$parent. menubar. file. menu menu sparent menubar.file. menu 1 Sactive_bg activeforeground $\$$ fg $।$ -background $\$$ bg - foreground $\$$ f $g$ ! - font sfont;

spare

File Menu state disabled - command (ine o I

sparent. menubar. file, menu add separator;

sparent.menubar.file.menu add command

-label Exit "
-underline 1 ।

170000 - comnand "destroy sparent";

pack

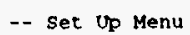

sparent menubar. ftle

-side left

- ipady 2;

bind sparent <Any-Focusin>

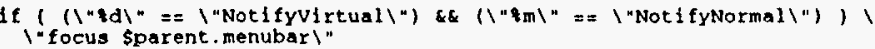

tk_menuBar \$parent.menubar
sparent. menubar. file:

turn sparent.menubar;

? Textwindow_createmenubar

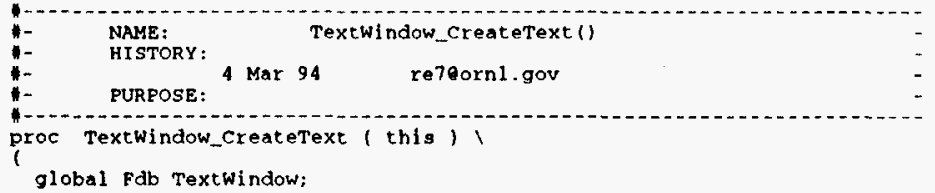

et parent \$Textwindow(\$this, TopWindow);

- - Frame for Text and Scrollbar

rame Sparent.text_f $z$ ame
-background STextwindow (Bg)

set text_widget sparent.text_frame. text;

- $\quad$ - Build Text widget

text stext-widget $\backslash$
background \$Textwindow(8g) । 


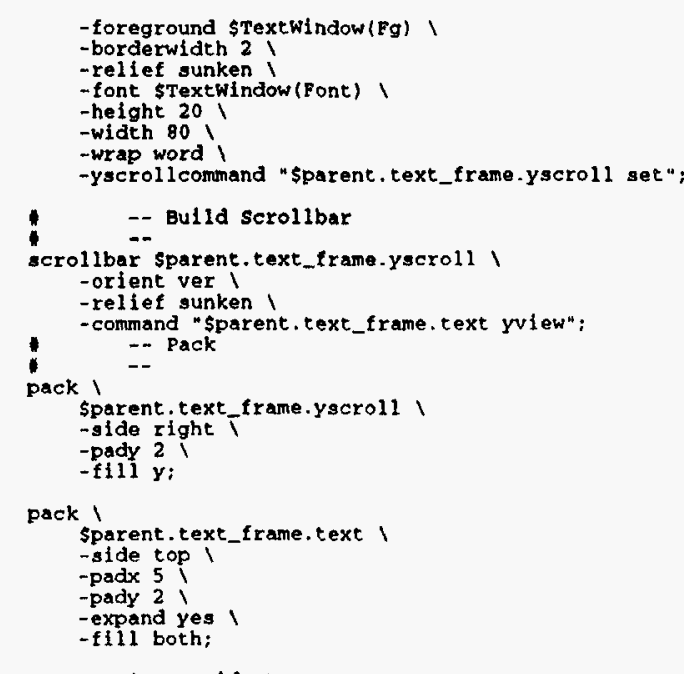

return stext_widget;

Textwindow_createrext

NAME:
HISTORY: 4 Mar $94 \quad$ rextwindow_Create(1)

proc Textwindow_create () 1

global Textwindow Fab_library;

- - - set Name of This object

set this cextwinstextwindow(count)

- -- Name of This window

set name twtopstextwindow (count):

* -- Build toplevel

toplevel Sname;
wm f conbi tmap sname SSFab_library/terminal $\times b_{m}$

mi iconmask sname @sFab_library/terminal.xbm

iconname sname "Text - sTextwindow(count)"

wm minsize Sname $1010 ;$
wm title sname "Text window - \$Textwindow (count)"

- - Buila Frames

rextwindow_createmenuar sname \$this;
set Textwindow(Sthis, Text) [Textwindow_CreateText \$this]

- -- Pack Frames

pack sname.menubar -side top -fill $x ;$
pack sname.text_frame -side top -ipadx 5 -ipady 2 -expand yes -fill both;
--: Increment window count

window (count):

return \$this

Textwindow_create

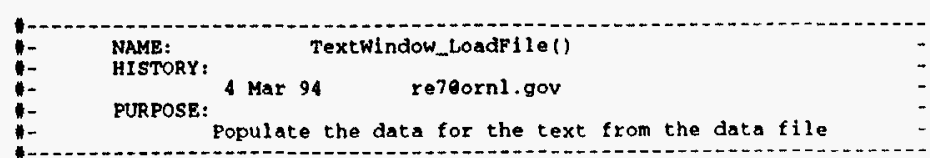

proc Textwindow Loadfile ( this fllename)

global Textwindow Fdb_l1brary;

set text_widget \$TextWindow(sth1s, Text)

-- open File

if ( leatch ( set fid lopen sfilename " $r$ ") 11$)$,

stext_widget insert end "Could not open file \$fllename";

else !

while ( [set wiath [gets sfid line)] > -1 )

if $($ swidth $>80$ ) ( stext_widget configure -width 132$)$;

stext_widget insert end [format "sisin" sline]:

update;

update

Textwindow_toadfile

NAME:
HISTORY: 4 Mar 94 rextwindow_Loadcommandresulti)
PURPOSE:
Populate the data for the rext from the network stream -
resulting from a command to the server

proc Textwindow_LoadCommandResult ( this fdb )

global Textwindow Fab_library:

set text widget sTextwindow (sthls, Text)

while ( "Iset width [sfab ReadNextLine line]1" ! = "eof")

if ( Swidth > Bo) ( stext_widget configure -width 132 ),

stext_widget insert. end [format " $s \backslash n$ " sline]:

update;

Textwindow_LoadCommandresult 


\section{APPENDIX B. WIDGET EXTENSION SOURCE LISTINGS}

\section{B.1 TKTHING.H}

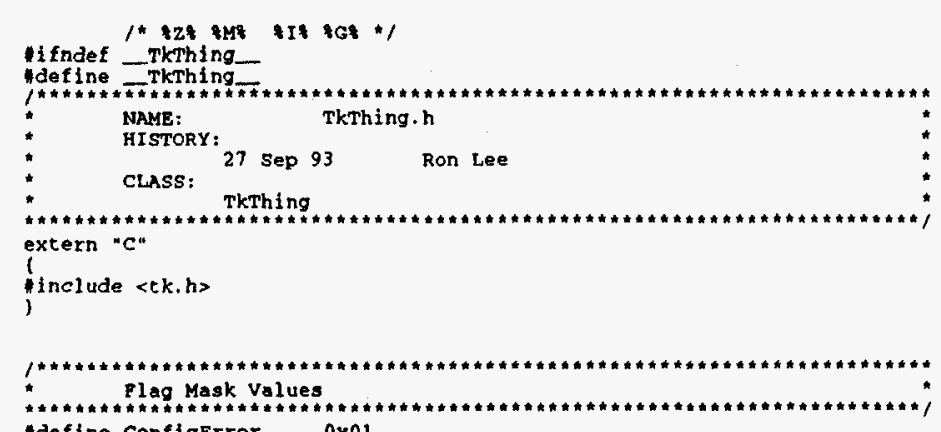
$\begin{array}{ll}\text { idefine configerror } & 0 \times 01 \\ \text { idefine RedrawPending } & 0 \times 02\end{array}$

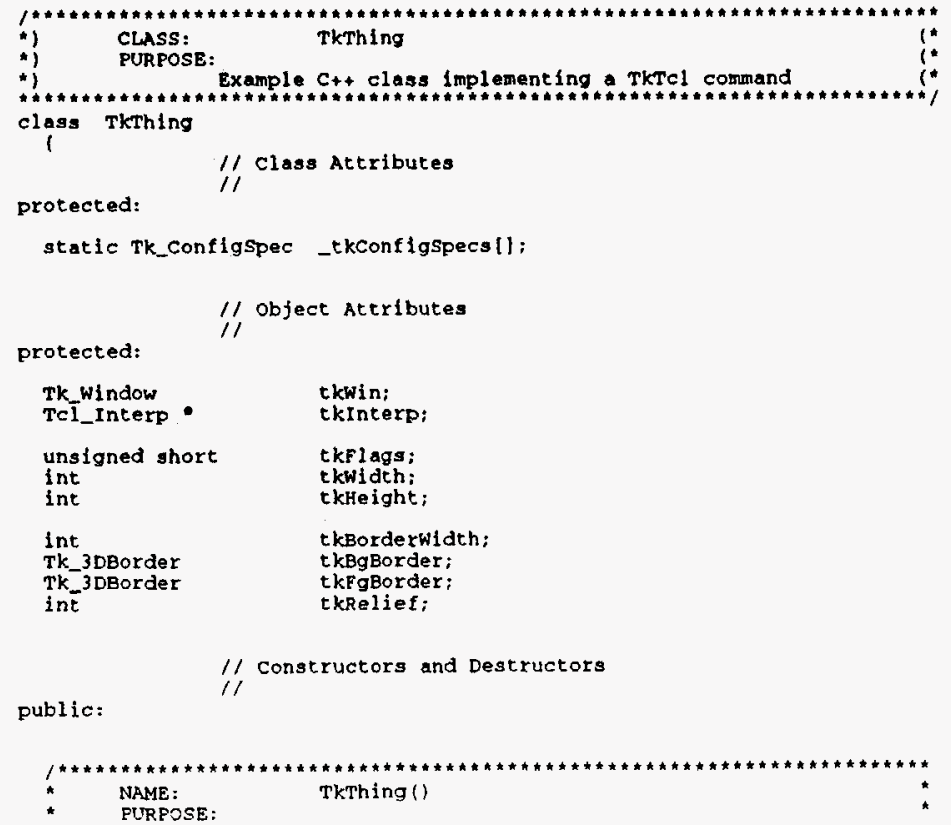

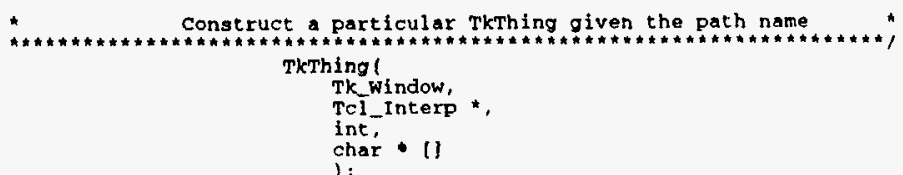




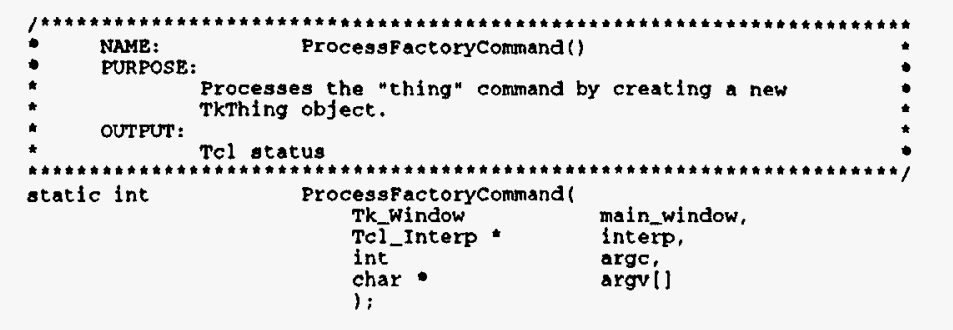

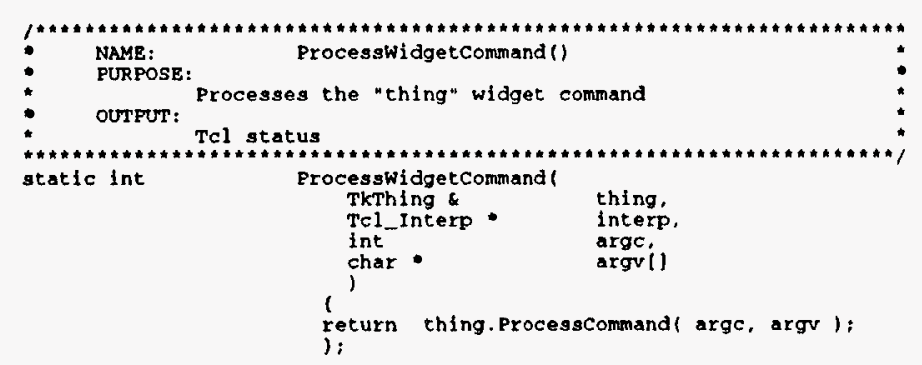

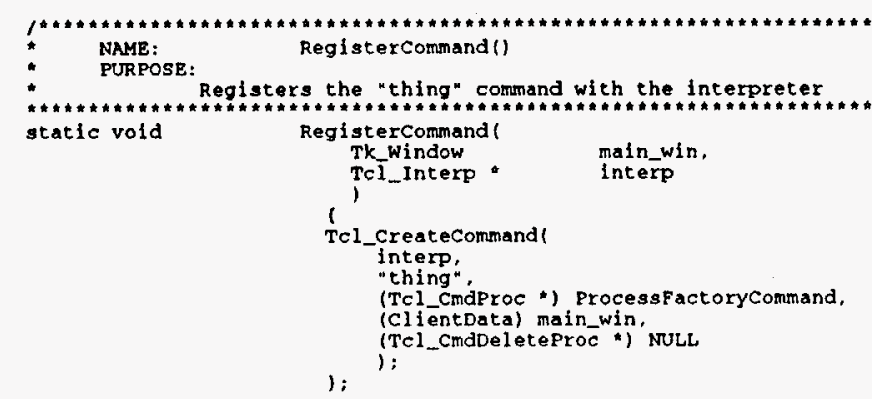

protected:

1/, Object Methods

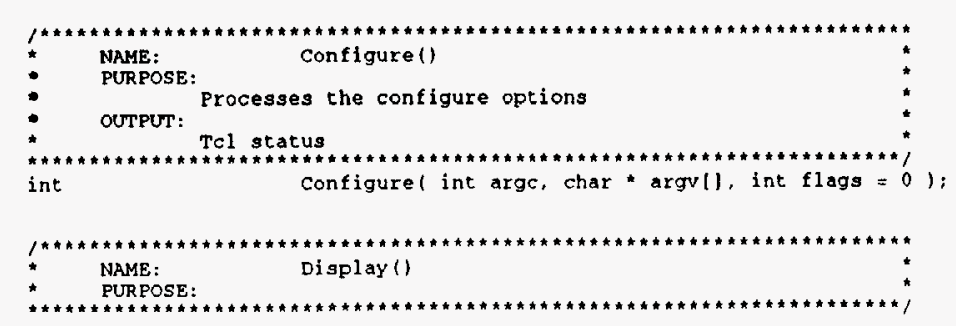

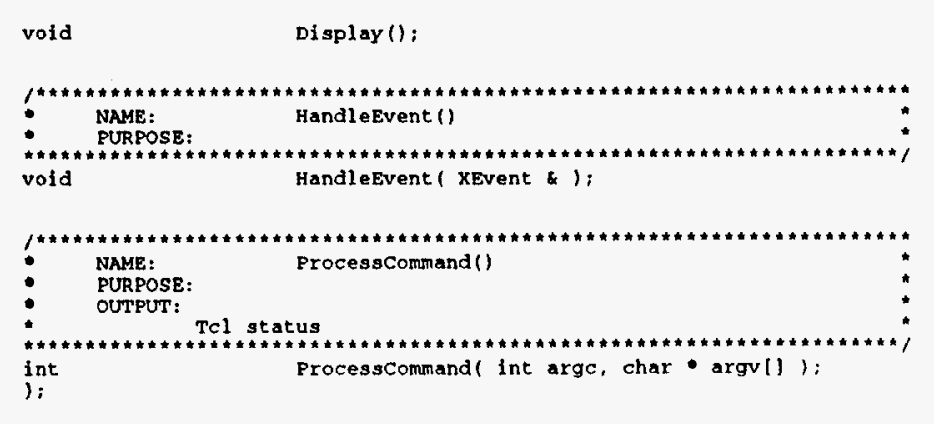

*endif 
Defaultscreen(Tk_Display(tkwin) )

TK_Fill 3DRectangler

Tk Display ( tkwin ),

tkBgBorder.

Tk_width ( tkwin), Tk Helght ( tkwin),

kBorderwidth threlie

xsetLineAttributesi

TK Dispiay ( tkwin),

gc.

Linesolid,

CapRound,

$x \operatorname{set} F$

Tk Display ( tkwin)

iTk_3DBordercolor( tkFgBordex )

Drawlinel

Tk_Display ( tkwin ),

gc.
of fset.

of tset.

tkWidth - offset

1,1 if

if

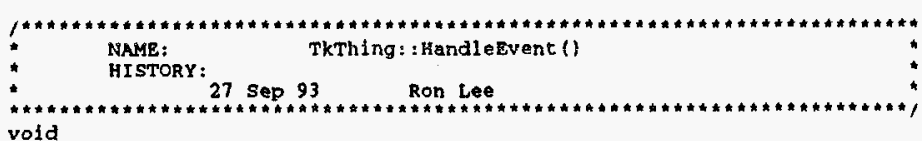

Toid TkThing: : HandleEvent( XEvent a event )

if ?

event. type $==$ ConfigureNotify $\|$

( event. type $==$ Expose $6 \&$
event. xexpose. count $==0$

'

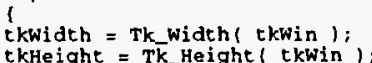

if ( 1 ( tKFlags \& RedrawPending) )

TK_DowhenIdle( Handledisplay, (ClientData) this )

tkFlags I I Redrawpending;

elge if ( event.type $=$ = Destroynotify )

TC1_Deletecommand ( tkInterp. Tk_PathName( tkwin ) )

if ( tkFlags \& RedrawPending )
Tk_Cancel Idlecall ( HandleDisplay, (ClientData) this);
Tk_Eventuallyfree( (ClientData) this, (TK_FreeProc *) Handledestroy); I// TkThing: : Handle Event

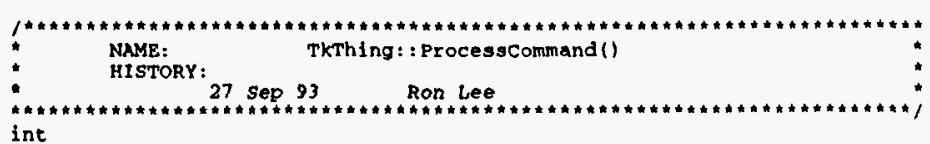

Tkthing: : Processcommand( int argc, char $*$ argv $[1$ ।

int result $=$ TCL_OK

if $($ arge $<2$ )

TCl_AppendRegult ( tkInterp, "Usage: ", argv(0), " option zarg $\ldots z "$, NULL );
result = TCL ERROR; result $=$ TCL_ERROR

else if ( strcmp(argul1\}, "configure") = =0

if 1 argc $=2$,

result $=T k_{\ldots}$ configureinfol

tkInterp.
tkwin.

tkconfigspecs

NULL,

)

i:

else if $(\operatorname{argc}=23)$

result $=\mathrm{Tk}$ _configureinfol

tkinterp
tkwin.

tkeonfigspecs.

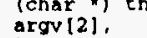

i:

else
result

return result:

NAME:
HISTORY:


If Check For Minimal Arguments

If $($ arge $<2)$ Tcl_SetRegult ( Interp, "Usage: thing pathname roptions?", TCL_VOLATILE ):
result = TCL_ERROR;

$1 /$ Create New Tk window

else if "

(new_win = Tk_createwindowfrompath( interp, main_win, argv[1], NULL 1$)==$ NeLL

Tcl_setResult ( interp, "Error creating window", TCL_VOLATILE ),

Yesult = TCL_ERROR;

// Create New object

else if

Tel_SetResult ( interp, "Error allocating data structure", TCL_VOLATILE) result

1/ Check Por Configure Error

else if ( new_thing->tkFlags \& Configerror)

Tk_Destroywindow( new_win ):

delete new thing;
result $=$ TCL_ERROR

else

Tk_Setclass( new_win, "Thing"):

Tk_CreateEventHandler

Exposuremask | structureNotifyMask.

(TkEventProe *) HandleEvents,

Tcl_Createcomnand

Interp Tkathame ( new win)

Tcl_CmdProc ") Processiwidgetcommand,

Tcl_SetResult( interp, TK_PathName( new_win), TCL_VoLATILE );

everything allocated

recurn result,

作 


\section{APPENDIX C. SOURCE LISTINGS FOR CANVAS ITEM EXTENSION}

\section{C.1 TKBARITEM.H}

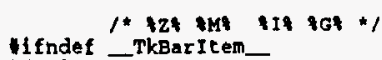

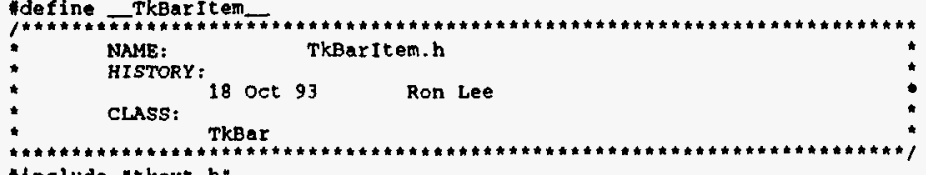

include "tkext. $h$ "

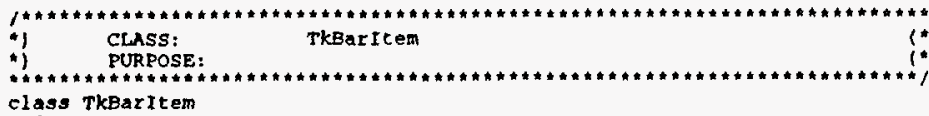

class TkBarltem

II Attributes

protected:

static Tk_configspec
static Tk_ttemType

public:

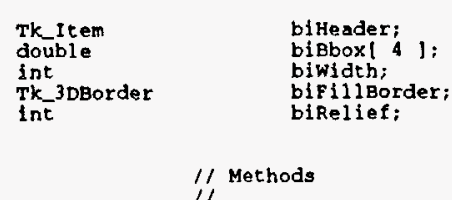

protected:

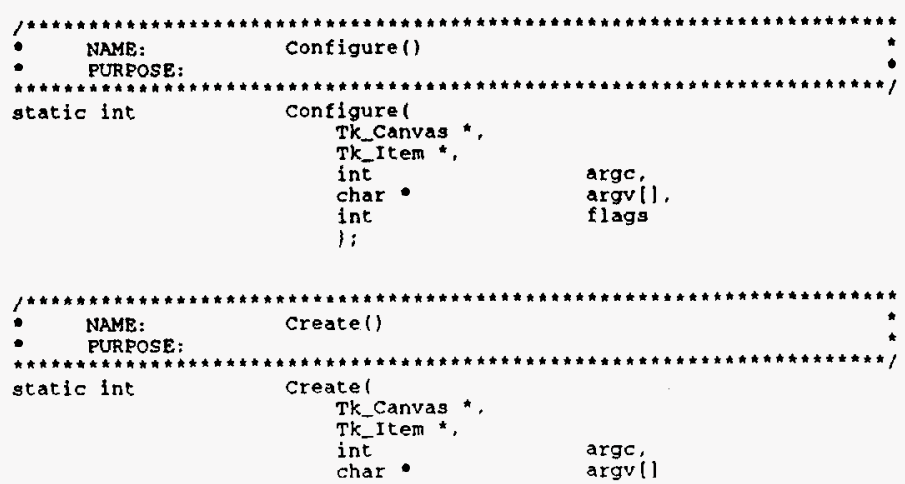

$1 ;$

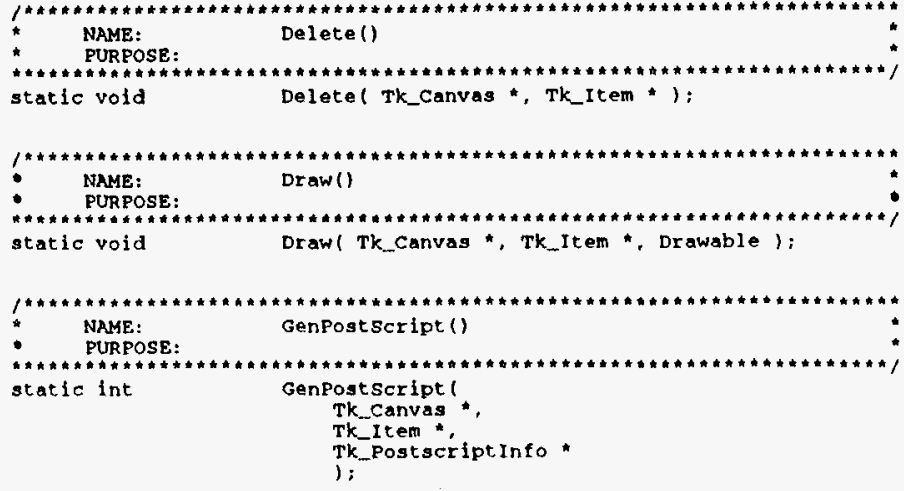

public:

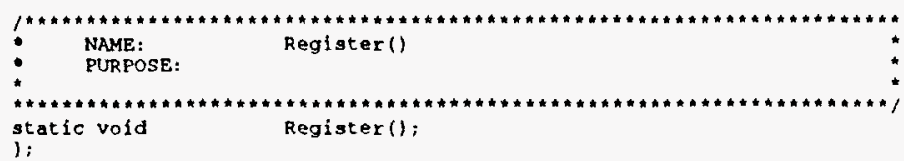

lendif 


\section{canvas ptr-stkwin, \\ argv: \\ (char ") bar_ptr \\ li:}

if $($ status $==\mathrm{TCL} O \mathrm{OK}$ )

(char *) bar_ptr)

Yeturn status;

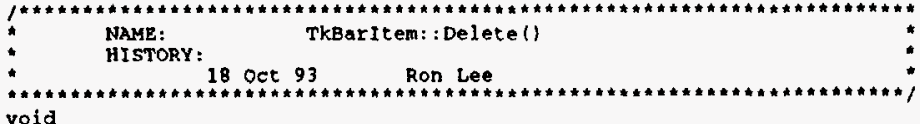

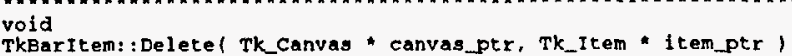

register TkBarItem * bar_pCr $=($ TkBarItem $)$ item_ptr:

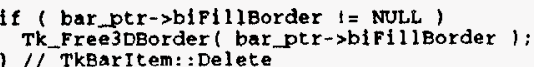

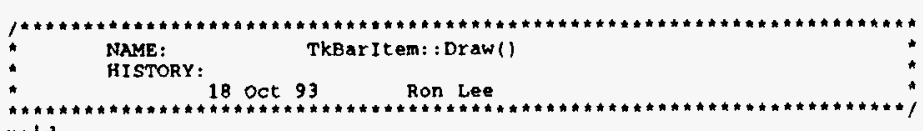$$
\text { vola }
$$$$
\text { Ron Lee } 93 \text { oct } 93 \text { R }
$$

Tkaritem: : Drawl

Tk_Canvas" "canvas_ptr.

Drawable drawable

' registex TkBarItem * bar_ptr = (TkBarItem *) item_ptr;

int

int
int
int

$x 1=$ sCREEN_X $($ canvas_ptr, bar_ptr->b1Bbox $(0)$ ):

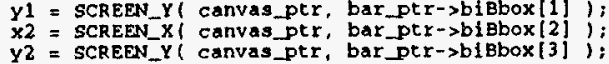

$1 /$ Must Be At Least 1 Pixel Big in Each Dimension

If $\begin{aligned} & x^{x} 2<=x 1 \\ & x^{2}=x^{2}+1 ;\end{aligned}$

if $\begin{aligned} & \left(y^{2}<=y^{1}\right. \\ & y^{2}=y^{1}+1 ;\end{aligned}$

TK_Fill30Rectanglel
canvas_ptr->display.

canvas_ptr->display,
drawable,
bar ptr->bifillBorder

$x 1, y 1$
$x_{2}-x i-1, y^{2}-y 1$

bar-ptr->biwidet,

1/ TkBaritem: : Draw

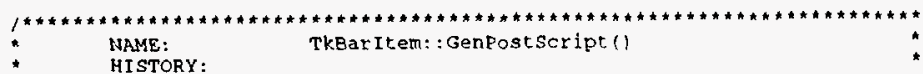

18 Oct 93 Ron Lee

TkBarItem: : Genpostscript

Tk Canvas?ostscript canvas_ptr,
TK Item.

TK_Item.

register TkBaritem * bar_ptr $=$ (TkBarItem
int status = TCL_OK; 1 tem_ptr;

$\begin{array}{ll}\text { char } & \text { path_emd } 500 \text { l } \\ \text { char } & \text { stringl } 100 \text { ]; }\end{array}$

double $\quad y 1=$ TkCanvpsy ( ps_info_ptr, bar_ptr->biBbox 11 )

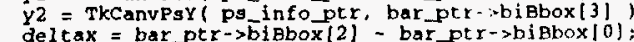

deltay $=y^{2}-y^{1}$

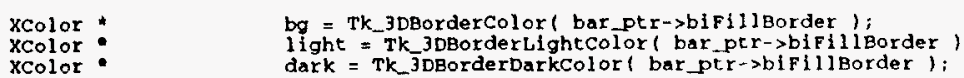

II Check colors

f $(\mathrm{bg}==$ NULL 11 light $==$ NULL 11 dark $==$ NULL $)$

else

I/ Draw Filled Rectangle

sprintfl

path_cmd,

"Ig ig moveto ig 0 rlineto o ig rlineto g neg 0 rlineto closepathin".

bar-ptr $\rightarrow$ bisboxiol, $Y 1$.

deltax,
deltay.
deltax

di:

Tcl_AppendResulc( canvas_ptr->interp, path_cmd, NULL );

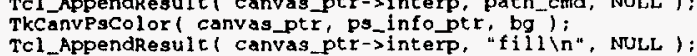

II Draw Bottom Shade Lines

sprintel

path_cmd,

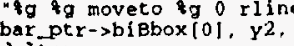

deltax,

baltay,

cl_AppendResult canvas_ptr $\rightarrow$ interp, path_cmd, Nuth

TkCanvPsColor 1

canvas_ptr.

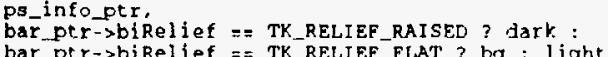

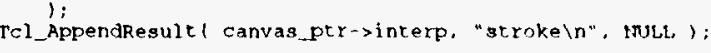
$1 /$ Draw Top Shade Lines

sprintf

path-cmd. 
bar_pty->biBbox $101, y 2$

bar deltay

bar_ptr $>$ biwidth

TRl_AppendResult ( canvas_ptr->interp, path_cmd, NuLL );

$$
\text { canvas_per. }
$$

gantinfo_pt

ar_ptr $\rightarrow>b i$ Rellef $==$ TK_RELIEP_RAISED $?$ light :

Tel_AppendResulte( canvas_ptr->interp, "strokeln", NULL ):

feturn status: // TkBaritem: : GenPost Script

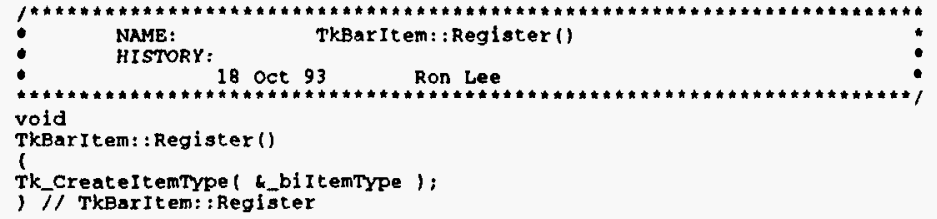




\section{APPENDIX D. SOURCE LISTINGS FOR PIXMAP EXTENSIONS}

\section{D.1 TKPIXMAP.H}

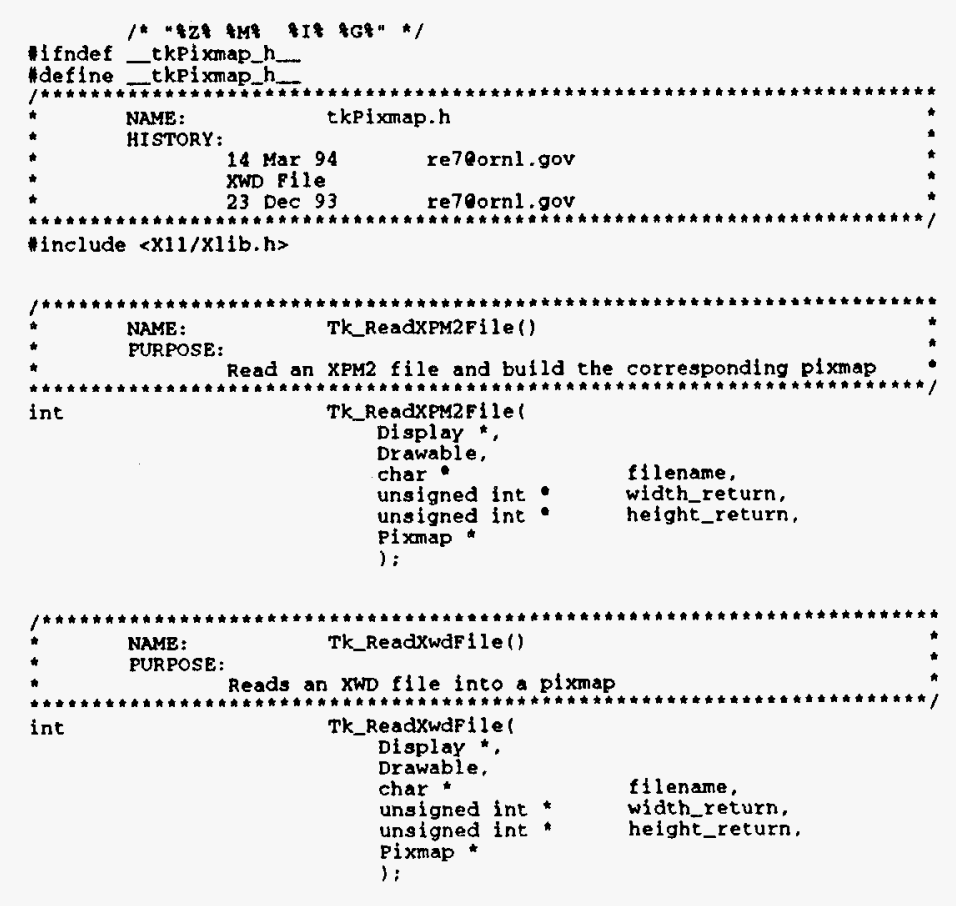

Mendif

\section{D.2 TKPIXMAP.C}

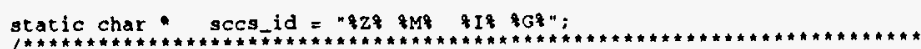

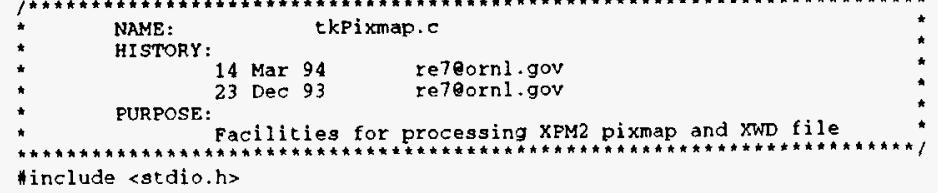

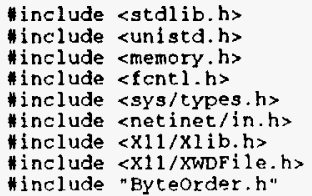
include <stdio.h> 


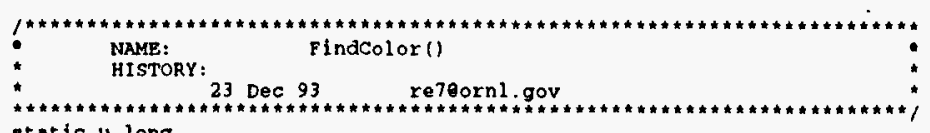

Findcolox ( xcolor - xeolors, int count, char ch)

int 1 ;

for $(i=0 ; 1<$ count $; i++)$

if $(\mathrm{ch}==$ (char) xcolors(i).pad

,

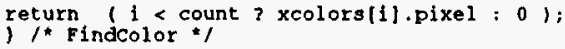

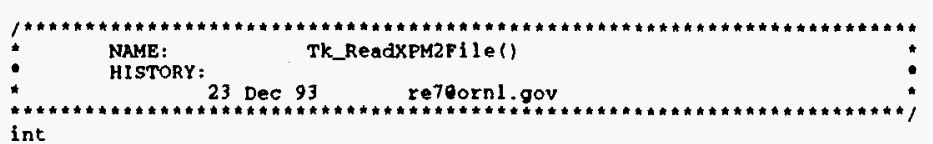

int

ReadXpM2Filel$$
\text { Drawable }
$$

igned int - width return,

unsigned int
pixmap

' in

FILE *

status = success;

GCCValues

xcolor*

int

fp;

ge:

xcolors;

, chars per pixel

rine [ 256 ]:

If ( display $==$ NoLL $)$
status = BadAccess;

else if ( drawable $==$ None
status = BadDrawable;

elge if ( (fp $=$ fopen ( tilename, " $r * 11)=$ NULL)

else

cmap = Defaultcolormap ( display, Defaultscreen ( display ) :

$l^{*}$ Look For Signature

if (fgets ( line, sizeof (1ine), fp $)= \pm$ NULL
status $=$ BadName:$$
\begin{aligned}
& \text { else if ( memcmp ( line, "( XPM2", } 6 \text { ) } \\
& \text { status = Badvalue; }
\end{aligned}
$$

status =

else
- Skip Comments, Read Parameters line

while (fgets( line, sizeof(line), $f p$ ))

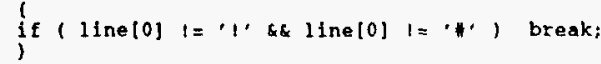

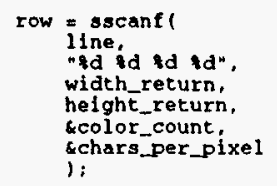

\section{". Check Parameters}

if ( row < 4 It chars_per_pixel $>1$,
status = Badvalue;

" Allocate xcolors Buffer

else if status a BadAlloc;

else

!"Read Color Descriptions

for ( row $=0$; row < color_count: rowt+

if (fgets ( line, sizeof(1ine), fp)

xeolors [ row ].pad a line $[0]$.

sscanfl

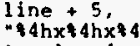

excolors! row i.red, sxcolors l row l.green
Excolors
i:

XAlloccolor ( display, cmap, xcolors + row

, 1 " for each color line *I

"Create Pixmap

"pixmap_return $=$ xcreatepi xmap 1

$$
\text { display, }
$$

"width_return

DefaultDepth ( display, Defaultscreen (display)

if ( "pixmap_return <= Lastextensionerror)

else

$$
\text { xsetfunction( display, gc, Gxcopy ): }
$$




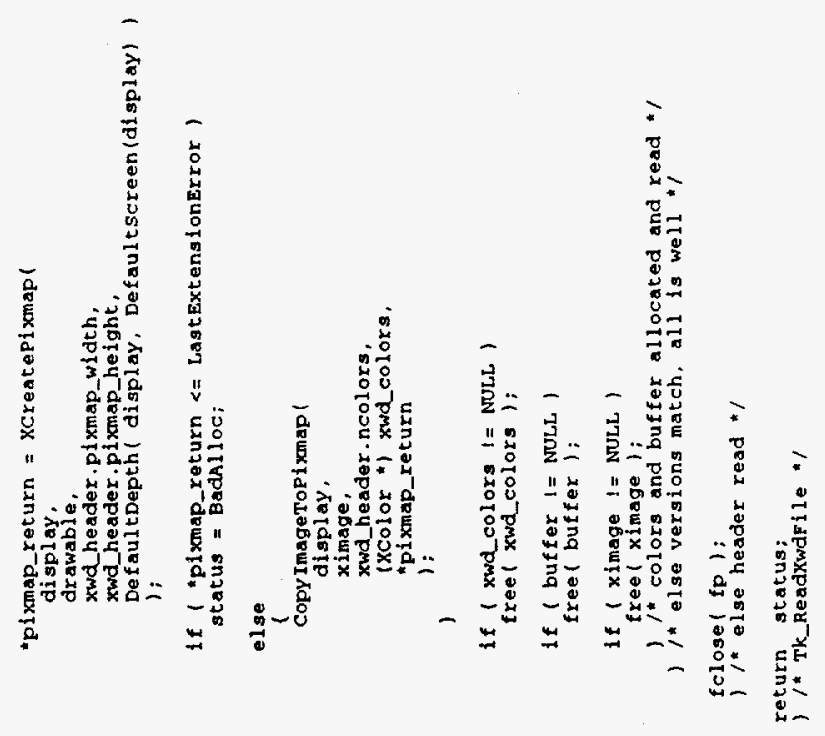

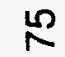

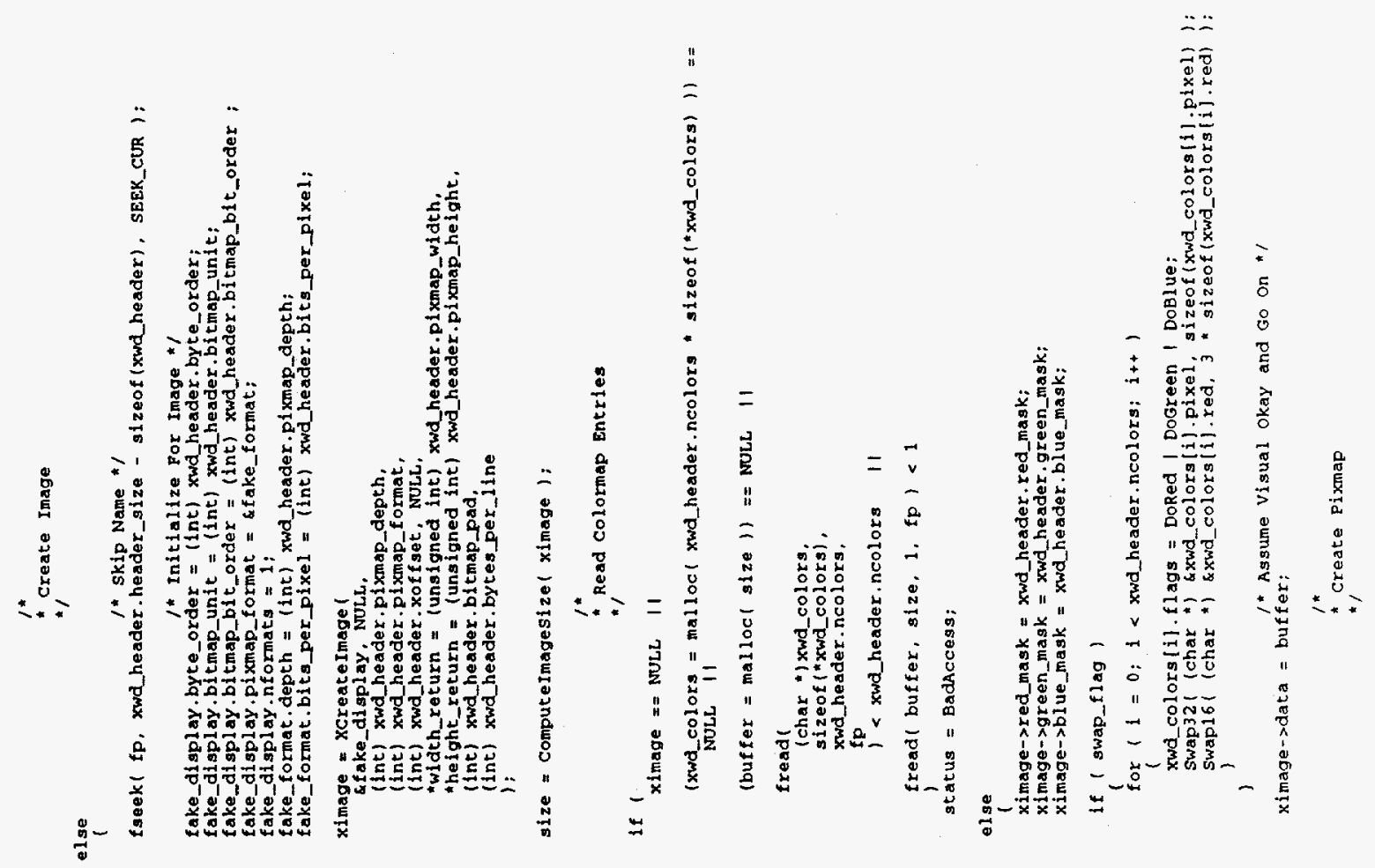




\section{D.3 TKBITMAP.C MODIFICATIONS}

Inelude "tkPi remap.h"

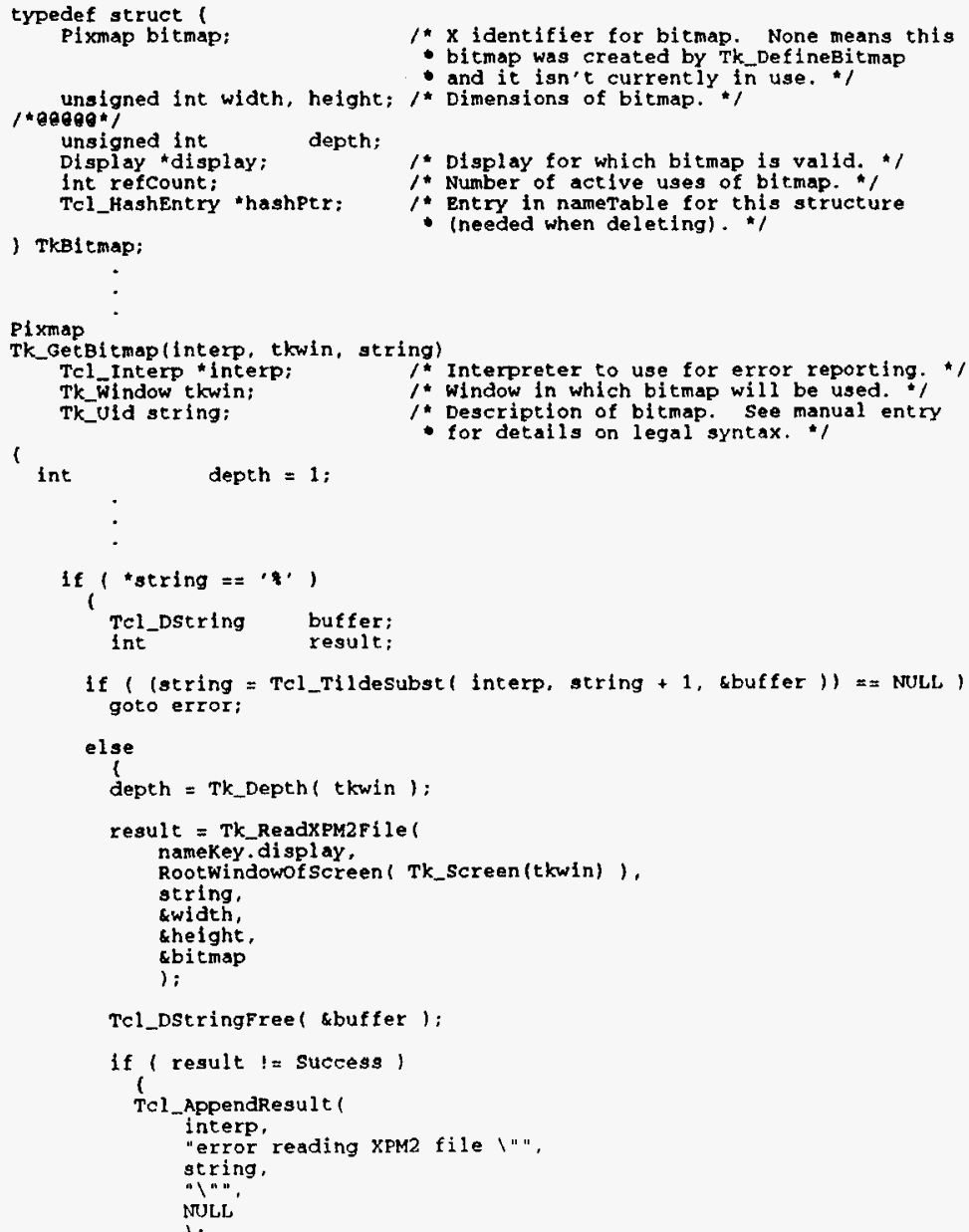

, 1 . if string begins with '.".

else if ( *string $=$ " $)$ )

$$
\begin{aligned}
& \text { TCl_Dstring buffer; } \\
& \text { int }
\end{aligned}
$$

if ( (string $=$ Tcl_Tildesubst ( interp, string +1 , sbuffer $)==$ NuLL)

else

depth $=$ Tk_Depth ( tkwin ):

result $=$ Tk_ReadXwdFilel

Rootwindowof Screen( Tk_screen(tkwin) ),

string,
swidth,

sheight,

$$
\text { ibi: }
$$

TCl_DstringFree ( sbuffer ):

if ( result $1=$ success $)$

Tcl_AppendResult ( "error reading xwo file !" ",

string.

NULL

goto error

$1 / *$ if string begins with * *

else if (*string $==$.e.) (

Tci_Dstring

string $=$ TCl_Tildesubst (interp, string +1 , sbuffer):
if (string $=$ NULL) (

Toto exror:

result = XReadBitmapFile (namekey, display, RootWindowofscreen (Tk_Screen (tkwin)),

if (result : = Bituapsucces)

Tcl_AppendResult (interp, "error reading bitmap file l" " string. goto error:

else

predefHashPtr $=T c 1$ FindHashEntry (\&predefTable, string)

Tcl_Appendresult (interp, "bitmap, " ", string.

goto error;

predefPtr = (FredefBitmap *) Tcl_GetHashValue (predofHashPtr): 
width $=$ predefPtr-> width;

height = predefPtr->height;

Wefaultrootwindow (namekey. display), predefptr->source. width, height)

Add Information about this bitmap to our database.

bitmappty = (TkBitmap *) ckalloc (sizeof (TkBitmap) ),

bitmapptr->bitmap = bitmap

bi imapptr->width $=$ width;

bitmapptr->depth $=$ depth

bitmapptr $\rightarrow$ display = namekey.display;

bitmapPtr->hashPtr = nameHashPtr

idkey.display = namekey.display;

12Hashptr = TCl_createHashEntry (sidTable, (chat ") sidkey,

if (Inew) (

if ('new) ('

TCl_SetHashvalue (nameHashptr, bitmapper):

Tc1_Sethashvalue (idHashPtr, bi tmapptr):

error:

urn None;

:

\section{D.4 TKBUTTON.C MODIFICATIONS}

static void

Display Button (clientData)

/* Information about widget. *"

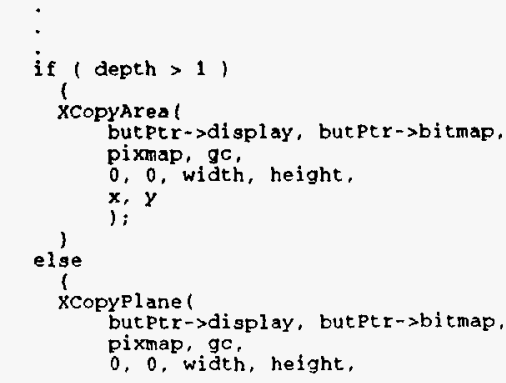




\section{D.5 TKCANVBMAP.C MODIFICATIONS}

static vold
DisplayBitmap

DisplayBitmap (canvasptr, itemptr, drawable)
register Tk Canvas "canvasptr;

Drawable drawable:

: Pitemap or window in which to draw
item.

register Bitmapitem *bmapptr $=($ Bitmapitem $*)$ itemptr

unsigned int

depth;

if (bmapptr->bitmap : = None)

TK_Di splay ( canvasPtr->tkwin),

bedepth

sdi:

if $($ depth $>1)$

XCopyAreal

Tk_Display ( canvasptr->tkwin ), bmappt $r$->bitmap, drawable,

(U_int) bmapptr->header. $\times 2$ - bmapptr->header. $\times 1$,

(u int) bmapptr->header. $y^{2}$ - bmapper->header bmapptr->header. $x 1$ - canvasptr->drawablexorigin.
bmaptr->heder. $y 1$ - canvaspt $x$->drawableyorigin

els

XCopyPl ane (TK_Display (canvasPt $r->t k w i n$ ), bmapPt $r->b i$ tmap, drawable, bmapptr->gc,
(unsigned int) bmapptr->header. $\times 2$ - bmapptr->header. $\times 1$,
(unsigned int) brapptr->header. $y 2$ - bmapptr-sheader. $y 1$, bmapptr->header. $x 1$ - canvasptr->drawablexorigin, 1)

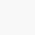
(

\section{D.6 TKMENU.C MODIFICATIONS}

static void

(splaymenu(clientData)

1" Information about widget. "/

$$
\text { if }(\text { depth }>1\}
$$

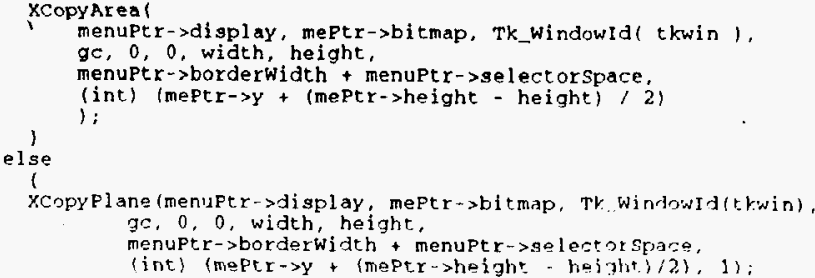




\section{D.7 TKMENUBUTTON.C MODIFICATIONS}

atatic vold

DisplayMenubutton (cl lentData)

i cilentData clientData;

/" Information about widget. "/

if $($ depth $>1$ )

mbPtr->display, mbPtr->bitmap, pixmap,

gc, 0,0, width, height, $x, y$

else

xcopypl ane (mbPtr->display, mbptr->bl tmap, pixmap.

) 


\section{APPENDIX E. SOURCE LISTINGS FOR C++ TCL CLASSES}

\section{E.1 TCLFDBCLIENT.H}

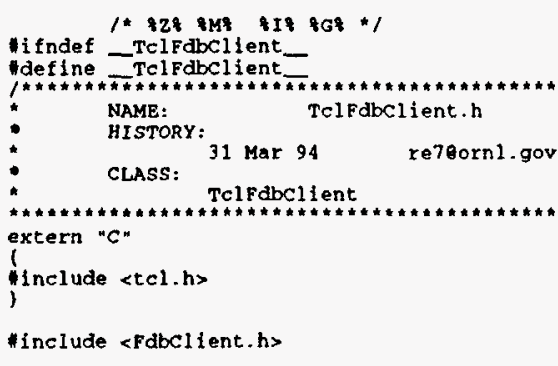

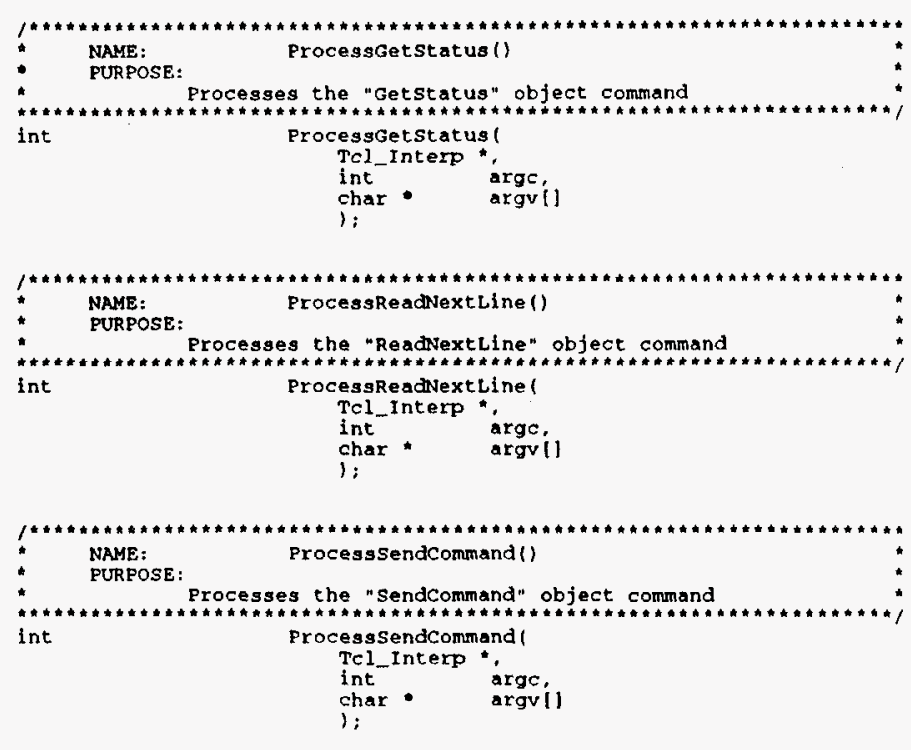

public:

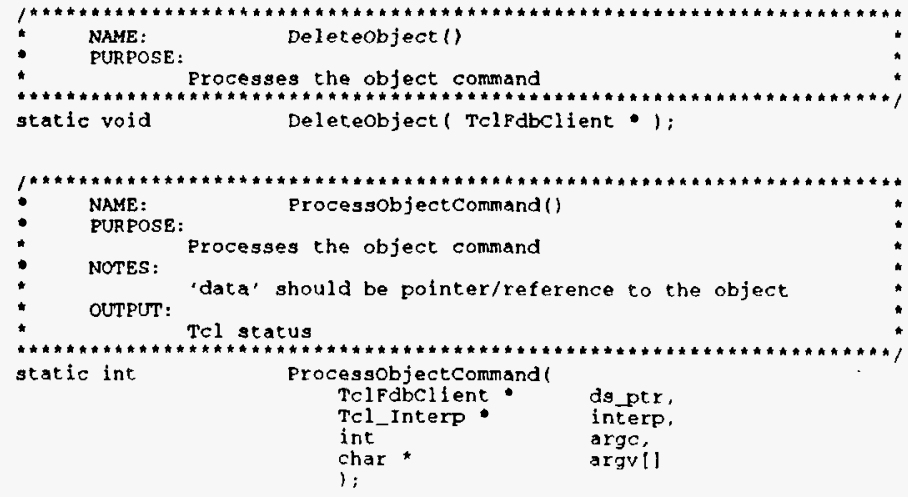




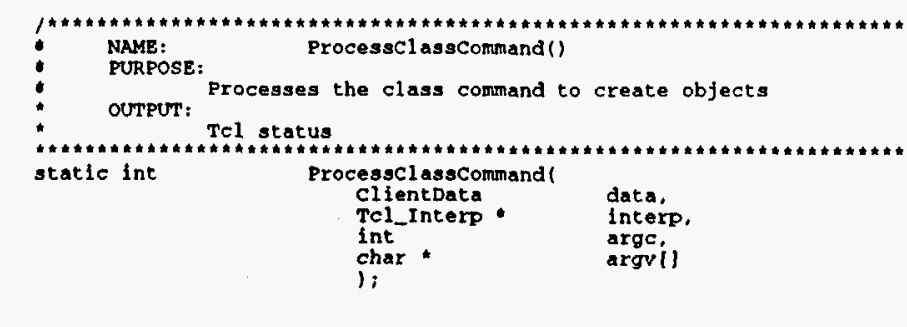

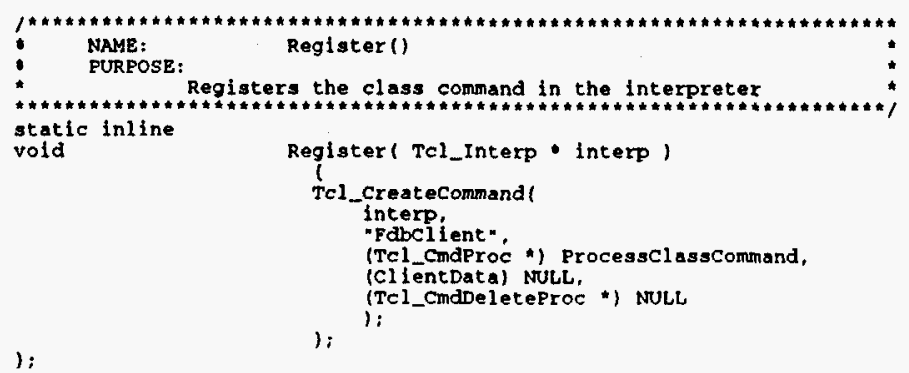

*endif

\section{E.2 TCLFDBCLIENT.CC}

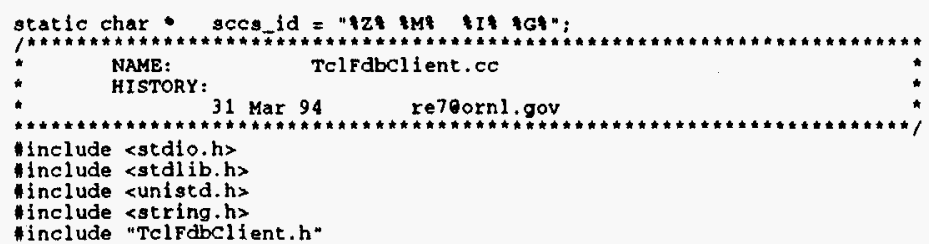

1/ TclFabclient: : TelFabclient

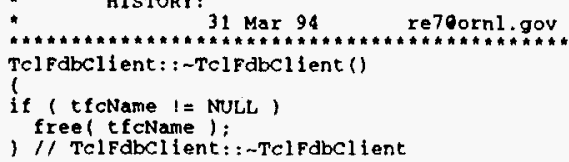


| // Telfabcl lent: : Processgetstatus

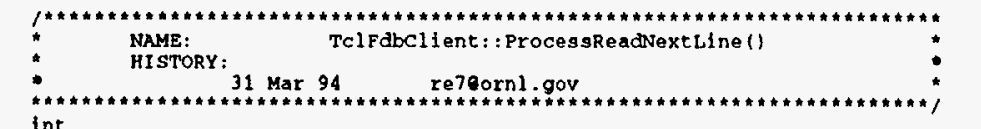

int
Tcl Fdbcllent: : ProcesskeadNextLine
Tcl_Interp interp,

$\begin{array}{ll}\text { Til_Interp - interp, } \\ \text { int } \\ \text { char } * & \text { argc } \\ & \text { argil }\end{array}$

I

int

Timeval next-1ine;

walt_time;

I/ check For varName Parameter

1f $(\operatorname{argc}<1)$

status $=$ TCL_ERROR;
TCl_SetResult

"Usterp: client ReadNextline varname ( wait-secs )", )

TCL_STATIC

else
if (argc $>1)$
wait_time $=$ Timeval( atoil argv(1), 0$)$,
ime_ptr = wait_time:

if ('next_line = (char *) ReadNextLinel time_ptr 1$)=$ = NuLL $)$

Tel_setvar( Interp, argvl01, "“, TCL_LLEAVE_ERR_MSG):
strapy ( buffer, "eof ");$$
\text { else }
$$

lse

TCl_setvar( finterp, argvio), next_line, TCL_LEAVE_ERR_MSG):

TC1 Setresult ( Interp, buffer, TCL_VoLATILE);

return status;

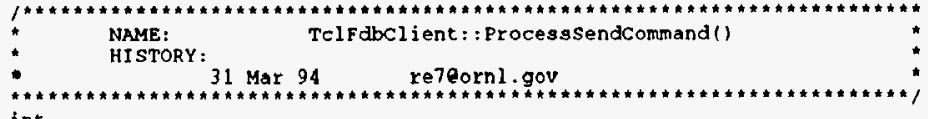

int Tclibabl ient: : Processsendcommand

$\begin{array}{ll}\text { Tcl_Interp } & \text { interp, } \\ \text { int } & \text { argc. } \\ \text { char } & \text { argvil }\end{array}$

' int

status $=$ TCL_OK: $\begin{array}{ll}\text { char } & \text { buffer } 32 \text { 3; } \\ \text { char } & \text { argsi fdbMaxArgs 1; } \\ \text { int } & \text { i: } \\ \text { int } & \text { send_status; }\end{array}$

1/ Check For Commandstring Parameter

if $(\operatorname{argc}<1)$

status $=$ TCL ERROR

TCI_SetResult (

"Usage: cl clent sendCommand command-string"

else

/1/ Parse Arguments

strtok( argvi0\}, " It" )

$1=0, \operatorname{args}[1]=\operatorname{strtok}($ NuLL, $"$ lt " $)$ args $|++1|=$ strtok $\left(\right.$ NULL, " $\left|\mathrm{t}^{"}\right|$

if ( (send_status = SendCommand (argv [0], args 1$)<0$ )
status = TCL_ERROR;

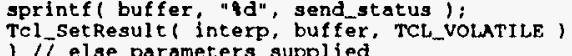

Jise parameters supplied

return status;

NAME:
HISTORY: $\quad$ TClFdbClient: : ProcessobjectCommand
int

int

Fabclient: : Procegsobjectcommand

TclFabclient ${ }_{\text {Tcl_Interp }}$ client_pt

$\begin{array}{ll}\text { int } & \text { arge } \\ \text { char. } & \text { argill }\end{array}$

in

status;

1/ Default Command is Getstatus

If 1 arge $<2$ '

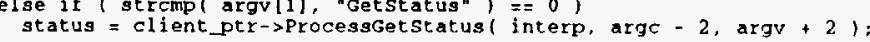

else if ( stremp (argv(1), "ReadNextLine" ) $=0$ )
status = client_ptr->processReadNextLine( interp, argc $-2, \operatorname{argv}+2$ );

else if ( strcmp( argv $\{1\}$, "Sendcommand" $=0$,
status $=$ client_ptr $\rightarrow$ Processsendcommand

arge -2 argv +21

-lse if ( strcmpl argv[1], "Delete" ) = = 1

status = TCL_or;
Tc1_Deletecommand interp, alient_pt $r$-st ferdame ): 
else

status $=$ TCL_ERROR

TC1_AppendResule

interp,

Sage: : In",

"lefabclient Getstatus' (returns status code) (n",

"Itrabclient Readvextline varname ( wait-secs ) (returns eof on Eof) (n". "ItFabcli ient SendCommand command-stringln".

return status;

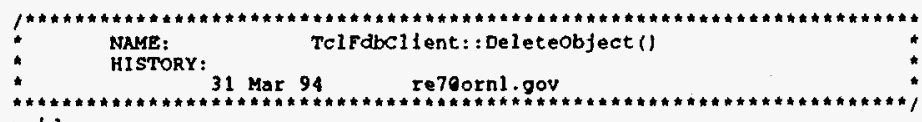

TclFabclient: : Deleteobject Tcl Pabclient * cllent_ptr

if (client ptr $i=$ NuLL )

, delete Client_ptr: Tclifdbclient: : Deleteobject

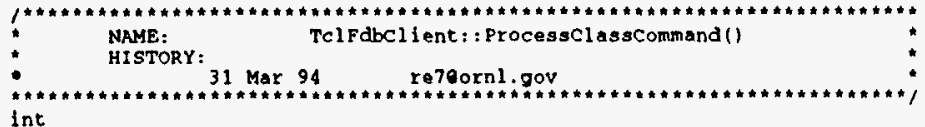

int TclFdbclient: : Frocesscl asscomnand

ClientData.

$\begin{array}{ll}\text { int } & \text { argc } \\ \text { char } & \text { argil }\end{array}$

i

$\begin{array}{ll}\text { int } & \text { status }=\text { TCL_OK; } \\ \text { TClFdbclient } & \text { new_item; }\end{array}$

atcen; 32 ,

// Check For Necessary Parameters

if $(\operatorname{argc}<4)$

TCl_Setresult

"Usterp: Fdbclient varname auth-id auth-key".

"Usage: Fdbel

statu' = TCL_ERROR;

// Process Arguments

else if 1 (n) 1

TCl_SetResult ( interp
status = TCL_ERROR;

else

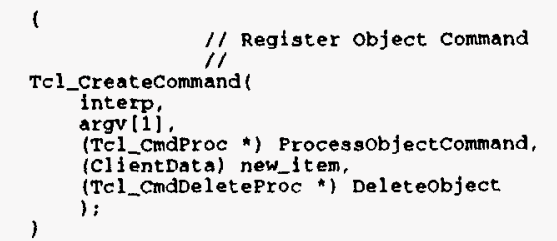

return status: 


\section{E.3 TCLSAMPLER.H}

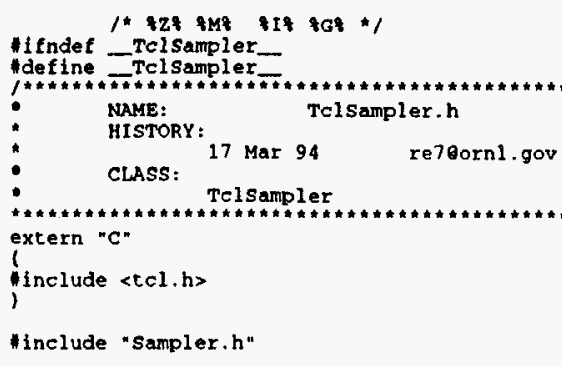

int

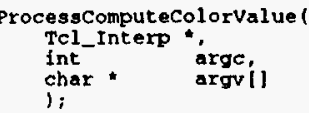

int

Processcreate日ing

$$
\begin{aligned}
& \text { Tcl_Intexp " } \\
& \text { int } \\
& \text { char } \\
& \text {,: argc, }
\end{aligned}
$$

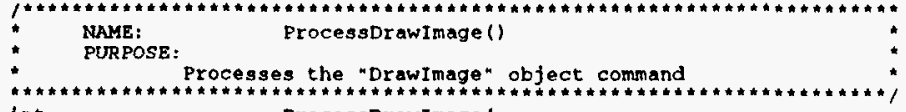

in

Tcl_Interp *

$$
\begin{aligned}
& \text { Tcl_Interp * } \\
& \text { int } \\
& \text { char } \\
& \text { i; arge, }
\end{aligned}
$$

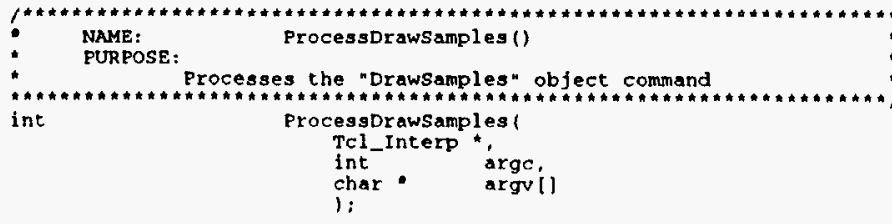

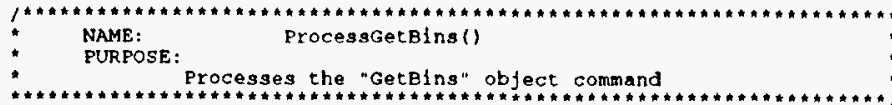

............... Processes the "Geteins" object comman

int ProcessGetbins?

$$
\begin{aligned}
& \text { Tel-Interp arge, } \\
& \text { int } \\
& \text { chat argul } \\
& \text { l: }
\end{aligned}
$$

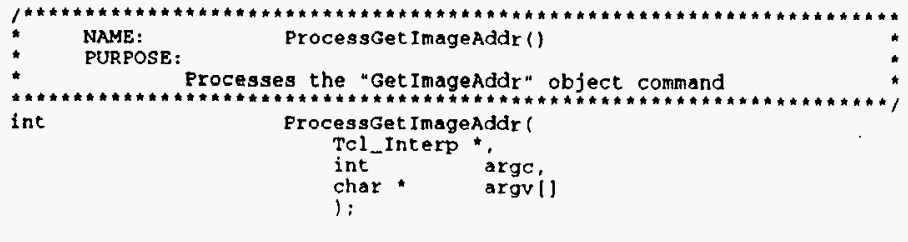




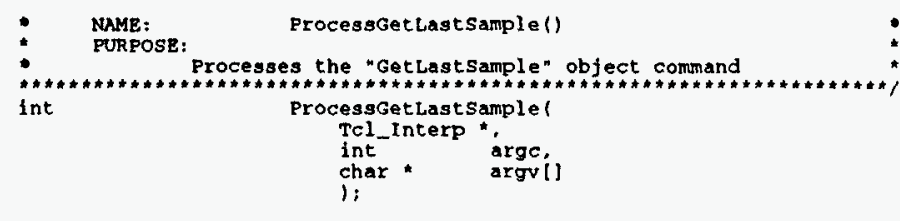

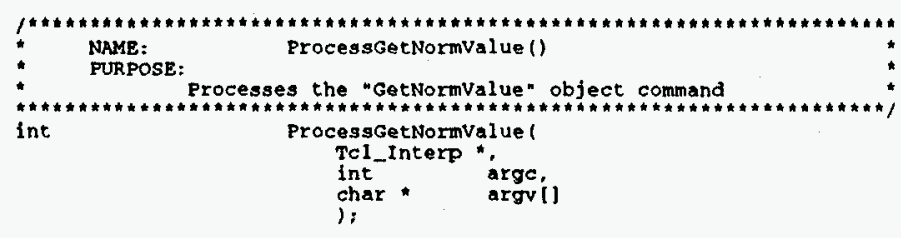

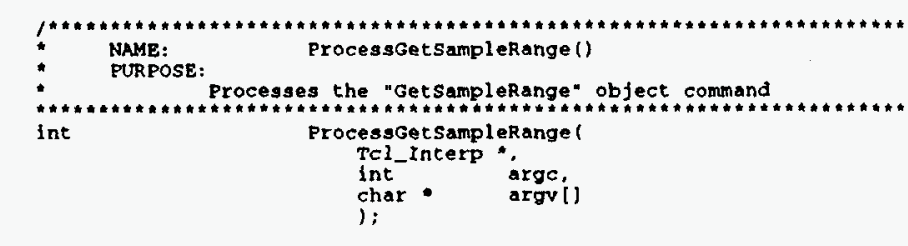




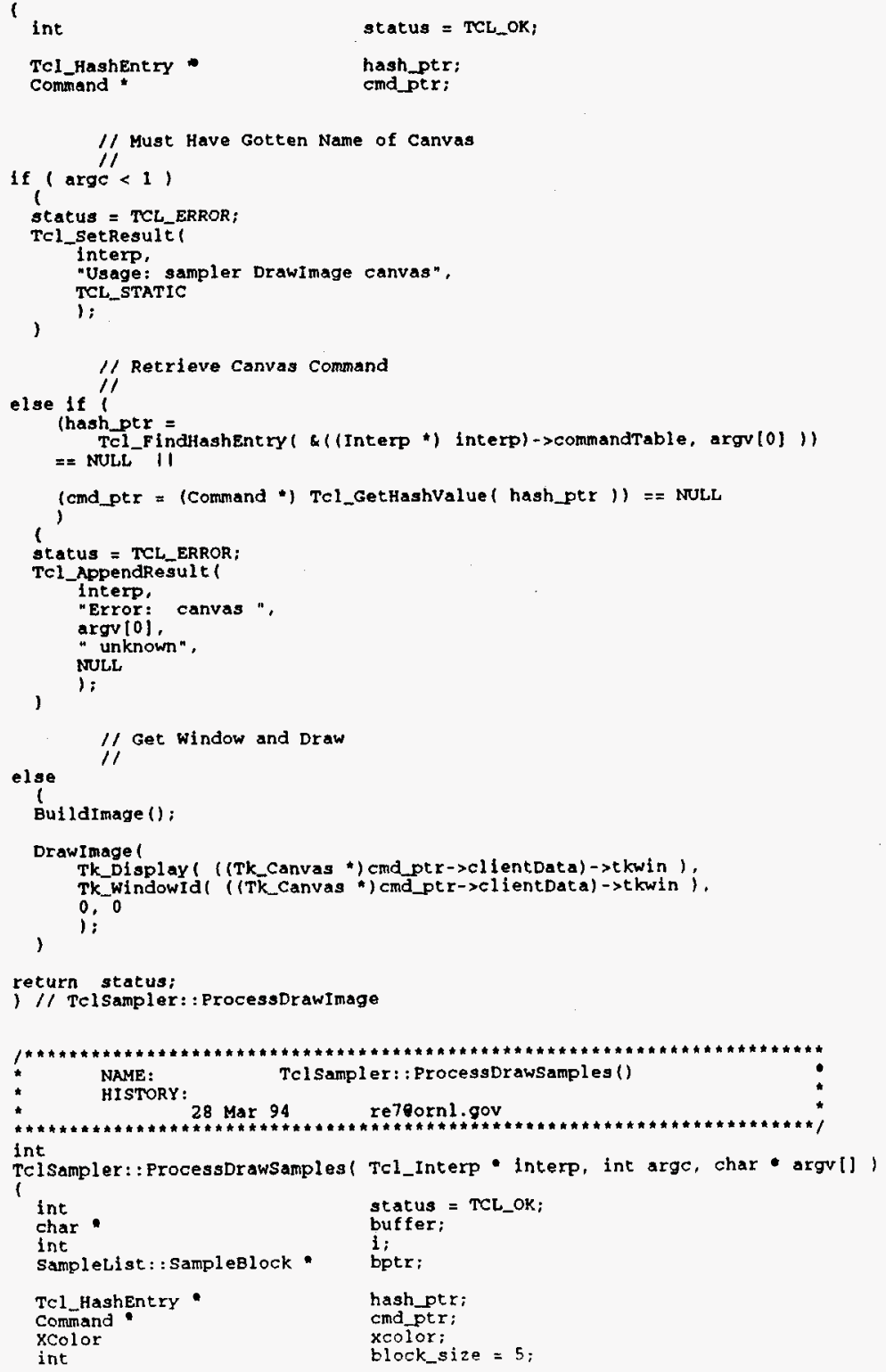

static char

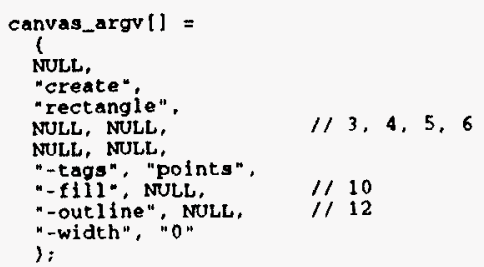

1/ Must Have Gotten Name of Canvas

if $(\operatorname{arge}<1$ )

status $=$ TCL_ERROR

"Ugage: sampler Drawsamples canvas [block-size]", ,

// Retrleve Canvas Command

else if ?

(hash_ptx $=$ TCl_FindHashentry (\& ((Interp *) incerp) ->commandTable, argv(0) )) $==$ NULL 11

(cmd_ptr = (Command *) TCl_GetHashvaluel hash_ptr 1$)==$ NULL

status $=$ TCL_ERROR

interp:

"Error:

unknown".

nuth

\section{// Allocate Strings For Parameters}

else if ?

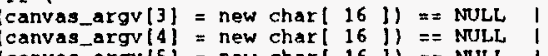
(canvas_argvis) = new char $(16$ i) $==$ NULL

Status $=$ TCL_ERROR;

II Execute Rectangle Create command For Each sample

else

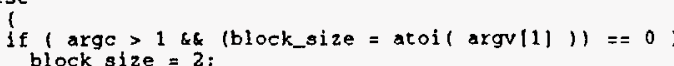

canvas_argv $[0]=\operatorname{argv}[0]$;

// Traverse Each Sample Block

for ${ }_{\text {bptr }}^{\prime}$ (sampleList:; Sampleblock *) ssamplesList.First.Tteml): 


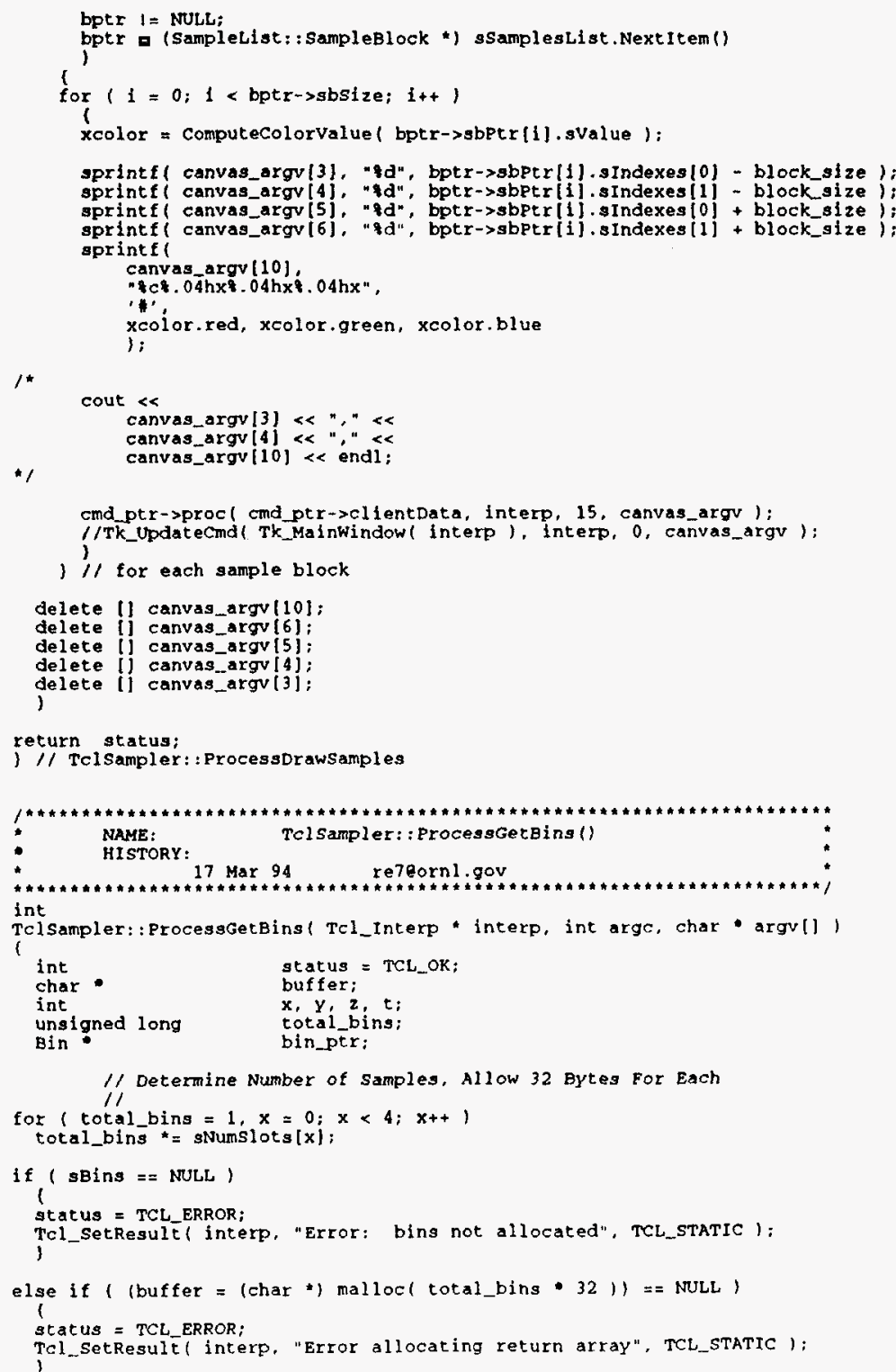

cmd_ptr-sproc ( cmd_ptr->cl lentData, Interp, 15, canvas_argv ): II for each sample block

else

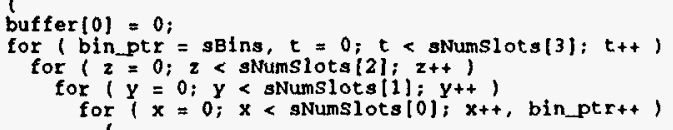

TC1_setResuit interp. buffer, TCL_DrNamIC ;

return status; i/ Tclsampler: : ProcessGetBins

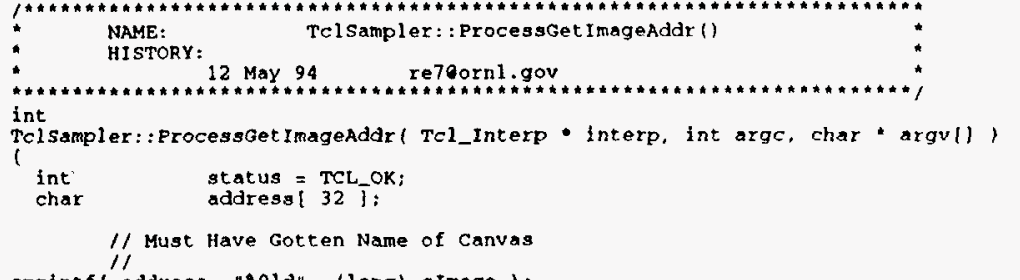

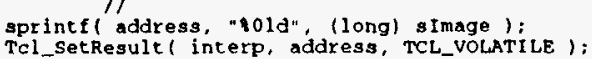

return status;

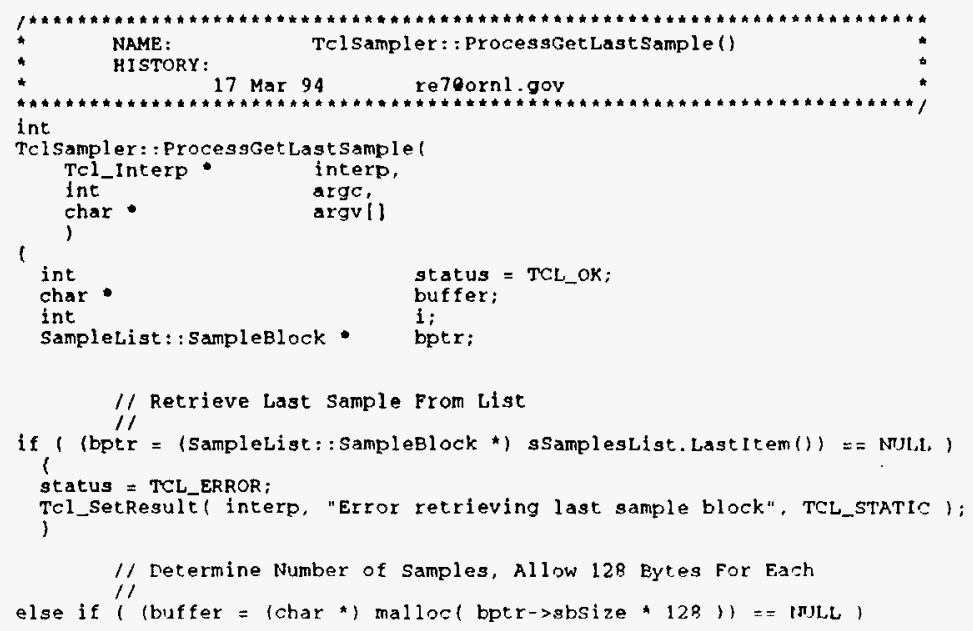




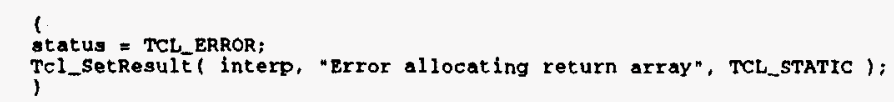

else

buffer $(0)=0$;

for $(1=0 ; 1$ <ptr->sbsize; it+

sprintfl

butfer, id id id thd tg)",

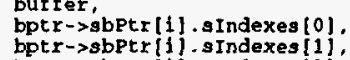

bpt $r>>$ sbptr
bptr $r>$ sbPtr $[1]$. sIndexes $(2)$,

bptr $\rightarrow$ sbPtr $(1)$. sTag,
bptr $>$ sbptr

,

TCl_SetResult ( interp, buffer, TCL_DYNamic):

return status;

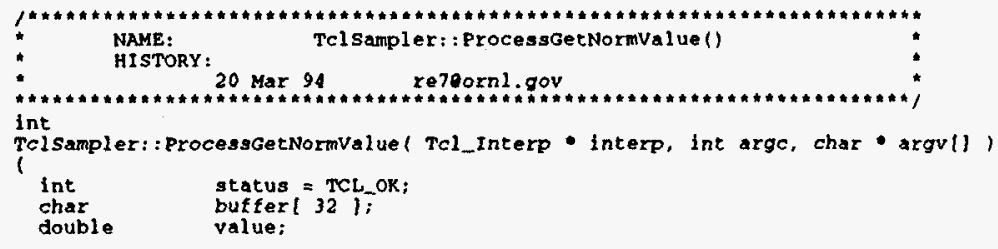

if $(\operatorname{argc}<1)$

status $=\pi)^{2}$ spoop

status = TCL_ERROR:
TC1_SetResulte interp, "Usage: sampler GetNormvalue value", TCL_STATIC ):

elige

value $=\operatorname{strtod}(\operatorname{argv}(0)$, NoLL $)$;

sprintfl
buffer

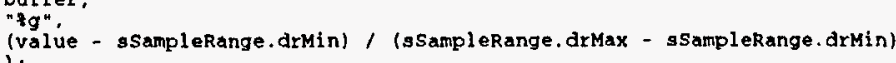

TCl_setresult ( interp, buffer, TCL_Volatile ):

return status;
I/ Telsampler: : ProcessGet Normvalue

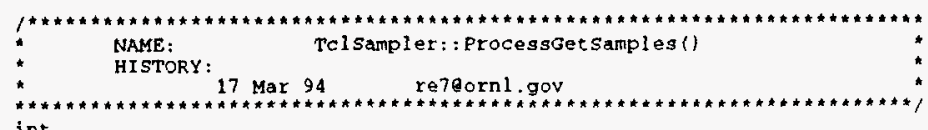

int TClsamplex: : ProcessGetSamples( TCl_Interp - interp, int argo, char - argul) , $\begin{array}{ll} & \text { status }=\text { TCL_OK; } \\ \text { int } & \text { buffer; } \\ \text { char } & \text { i; } \\ \text { int } & \text { bptr; } \\ \text { SampleLl st : : SampleBlock * } & \text { b }\end{array}$

I/ Determine Number of Samples. Allow 128 Bytes For Each

if ( (buffer = (char ") malloc (stotalsamples - 128 ") = NULL)

status = TCL_ERROR;
TCl_SetResult i interp, "Error allocating return array", TCL_STATIC,$$
\text { else }
$$

buffer $101=0$;

for 1

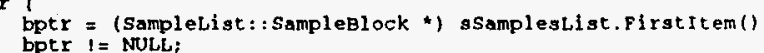

bptr I= NULL;
bptr = (sampleList: : SampleBlock ") sSamplesLigt. Next Item()

for $11=0 ; 1<$ bpt $x->$ sbsize; $1++1$

sprintf (

buffer id ad id and ig I",

butfer,
bptr-sbptrli].sindexes 10$)$.

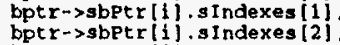

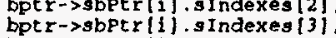

bptr $\rightarrow$ sbptr $\{1\} . s T a g$
bptr $\rightarrow>$ sbptr

1

TCl_setresult ( interp, buffer, TCL_DYNamic),

return status:

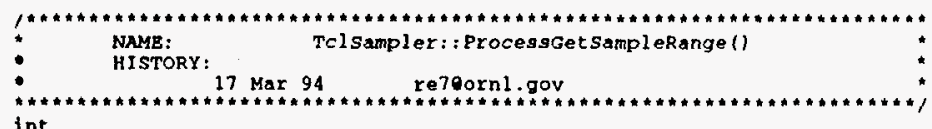

int Tclsampler: : ProcessGetsampleRange

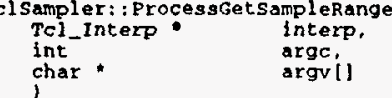

'int

atatus $=$ TCL_OK
buffer $(64) ;$

sprintf( buffer, "g gg", ssamplerange.drMin, ssamplerange.drMax ।:

TC1_SetResulte (interp, buffer, TCL_vOLATILE);

return status;

Processget sampleRange 


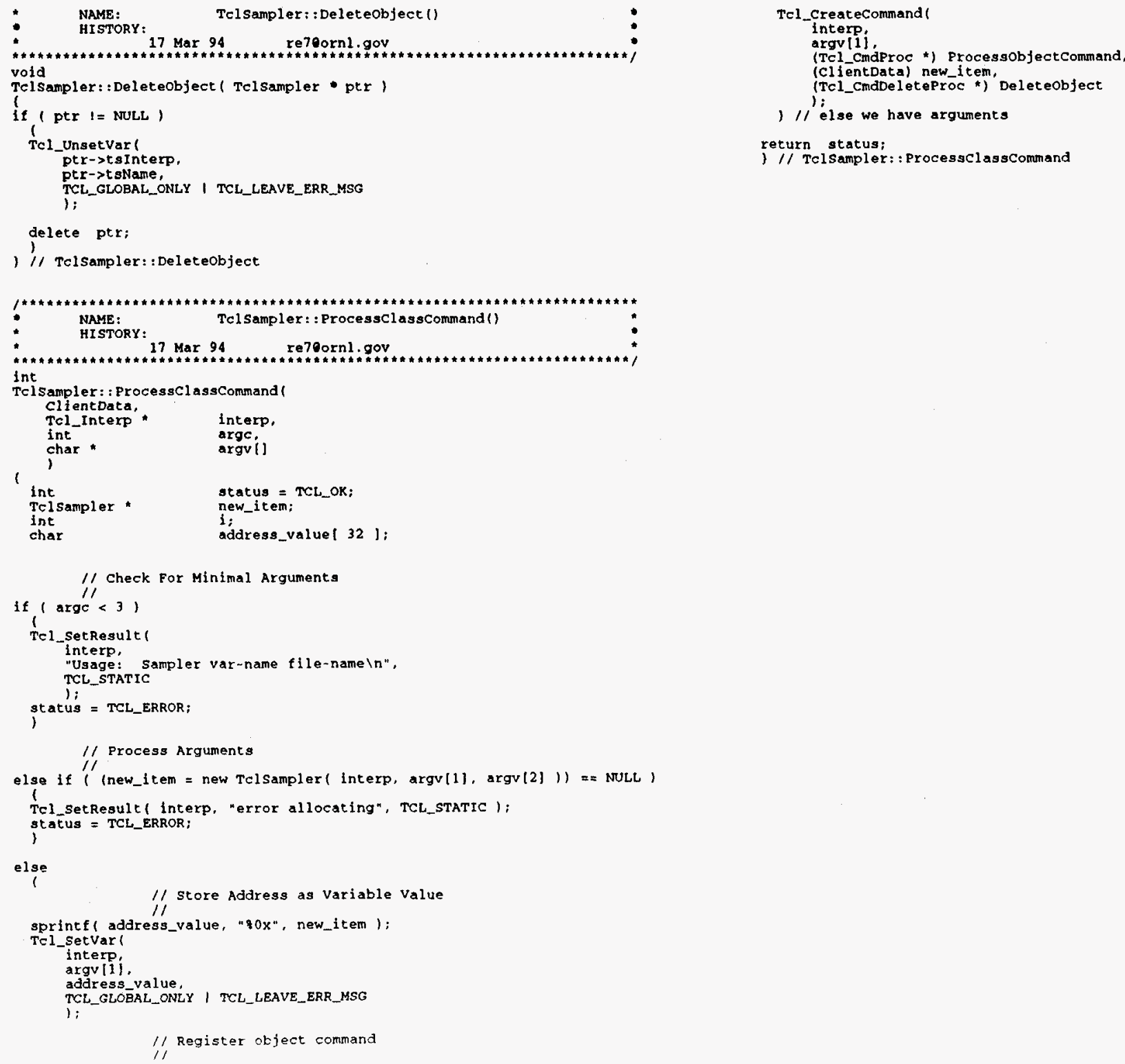

Tcl_createcommand$$
\text { interp; }
$$

argv [1], diproc *) Processobjectcommand (ClientData) new_item,
(Tcl_cmadel leteProc *) Deleceobject

$1 /$ else we have arguments

return status; 
$1 /$ Open Display

else if ( (display = XOpendisplay ( NULL ) =e NULL)
cerr \&< "Exror: could not open displayin";

else

screen = Defaultscreen ( display ),

$/ /$ Create window

xwin.createl

Rootwindow( display, screen)

100,100,
400,400

xwin. Definecursor( Xcreatefontcursor( display, Xc_iron_cross ) )

xwin. SetwMProps \{

argv [0],

xcreateBit tmapfrombatal

display inindow()

xlogo 32 bits.
xlogo32_width.

\section{Select Input and Map}

xwin. selectinput

ssMask | ButtonReleaseMask | Exposuremask | structureNotifyMask xwin', Map ()

if 1 11 Open xwa file

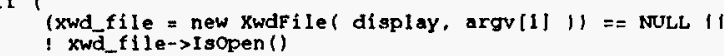

cerr « "Error: could not read xwd file " « argul1] « " In"

else if 1

$$
\text { // Allocate Point Array }
$$

(points = new ImagePoint)

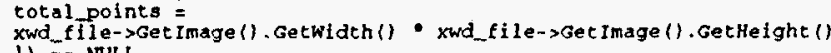

1) $==$ NuLL

cerr « "Error: could not allocate point arrayın";

else

Imagepoint
Imagepoint

// Read source Image values

cout « "reading source image " « argv|11 « " ... In";

$y=0$, counter $=0$, data_ptr $=$ xwd_file $>$ Get Image() . GetData ()

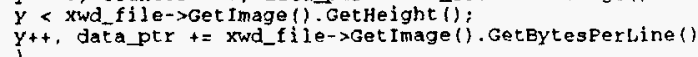

for $(x=0 ; x<x w d$ file- $x$ GetImage (). Getwidth (): $x++$ ) cout « "read completeln";

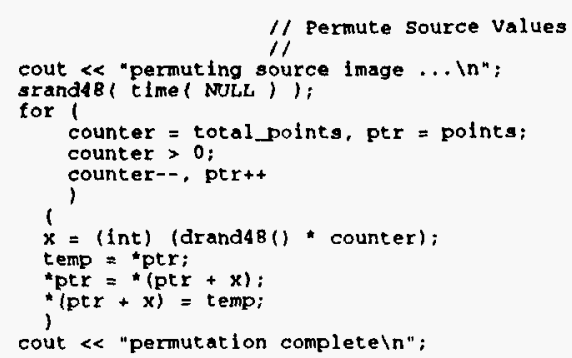

// Create sample Image

ample_image. Createl

xwd file->Get Image () . Getwidth(),

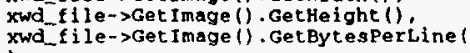

if (' l sample imege iscreatedi)

else

1

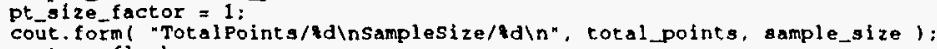

cout \&\& flush:

Readsamplel

points.

sample_image
total_points

sample_size,

xwin. Regizel

Xwd_ti le->Get Image (). Getwidth (),

XFlush( display );

for ( finished = False; Ifinished;

'fd_set read_fds:

FD_ZERO (

FD_SET( display->fd, \&read_fds),

switch

select (getdtablesize(), sread_fds, MULL, NULL, NULL ) 1

case -1:

perror( "select() error" ), 
case 0:
break;

default:

1 (

XNextEvent (display, kevent )

if 1

(event.type $==$ Expose || event. type $==$ Configurenotify $\mid$ event. xexpose. Window =

sample_image. Put( xwin.Getwindow() );

else if (FD_ISSET( O. Gread_fds ),

char line 80 \};

char
int

if ( fgets( line, sizeof(line), stdin) )

if ( Itoken = strchr( 11ne, ' $/ 1):$ = NULL)

if ( sscanf( line, "ld" thew_size) $>0$
sample_size = new_size;

if ( sscanf( token, "qd", knew_size) $>0$ )

If I samplessize $=0$ )

else

Readsampler

points.

sample image,

samplessize,

pt_size-factor

,

IIIswitch

II elor

II else Xwdiple object and Imagepoints allocated

delete $x_{\text {I }}$ file 


\section{F.2 PERMUTEVIEW.TCL}

1/ ugr/local/bin/wish -f

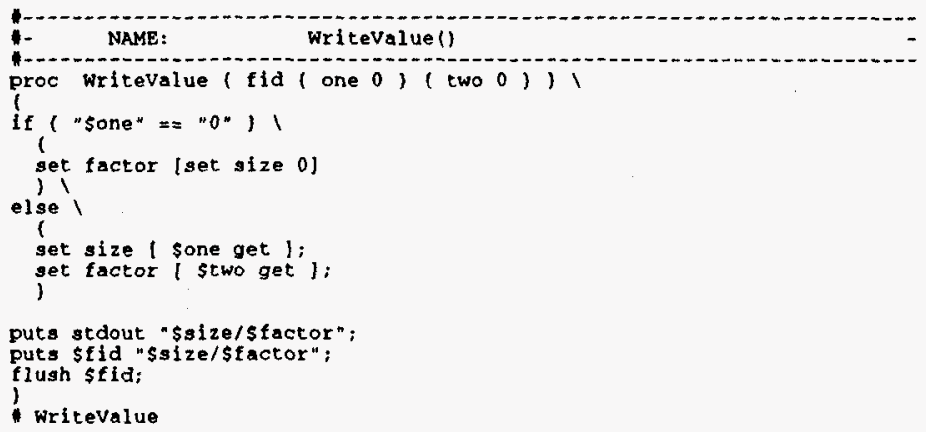

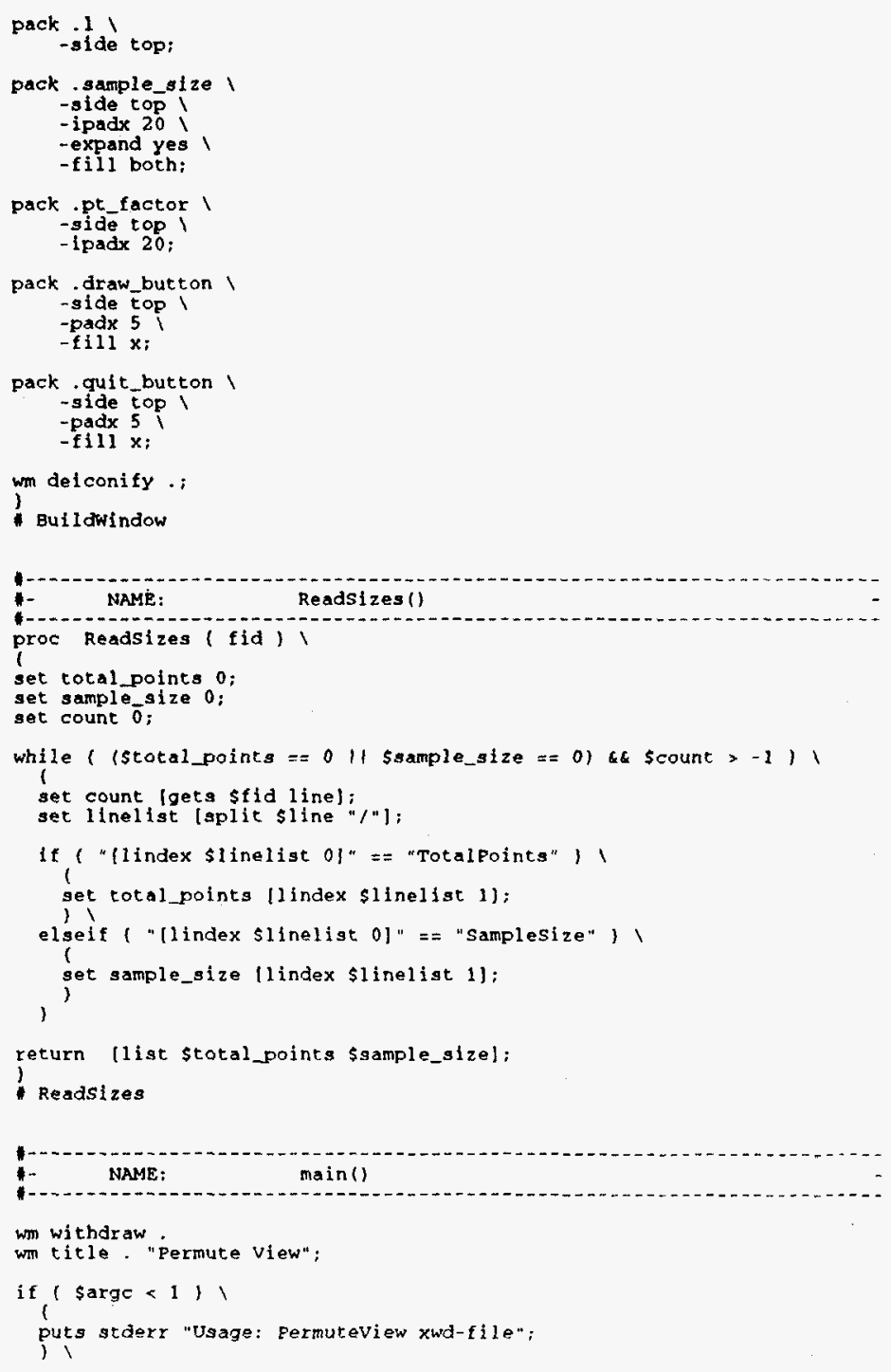

return [1ist stotal_points \$sample_size],

Readsizes

wm withdraw "Permute view";
wit title. "Pame:
if ( sarge < 1 ) ।
puts stderr "Usage: germuteview xwd-file";


else 1

set cmd "I/usr2/sre/xobjects/permute_view [11ndex sargv 01";

if ( (catch ( set xplpe lopen scmd " $r+n$ ) )) ।

puts stderr "Error spawning permute_view"

else'

set value_list [Readsizes \$xplpe].

puts stdout "... \$value_list";

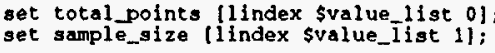

if ( stotal_points $=0$ ll ssample_size $==0$ )

puts stderr "Brror reading from permute-view":

else'

Buildwindow sxpipe stotal_points ssample_size 


\section{APPENDIX G. SOURCE LISTINGS FOR OBJECT-ORIENTED TCL CONSTRUCTS}

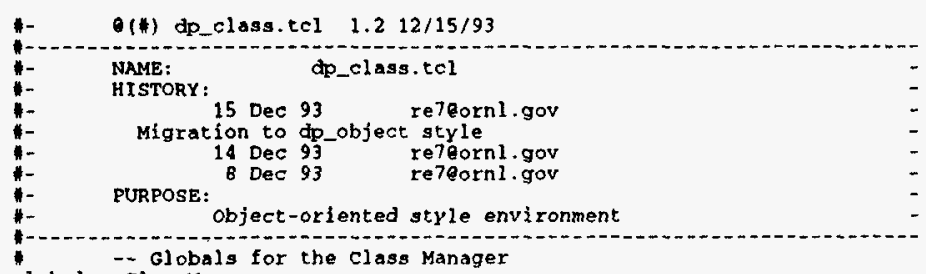

global classmgr.

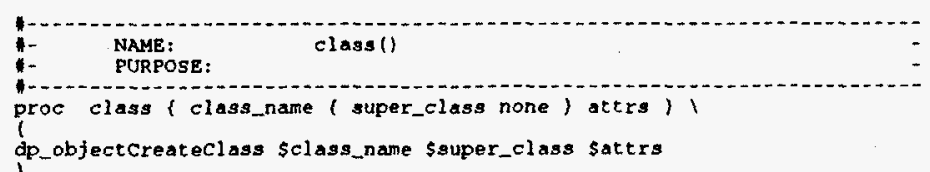

dp abjectcreateclags sclass name ssuper_class sattro

I class

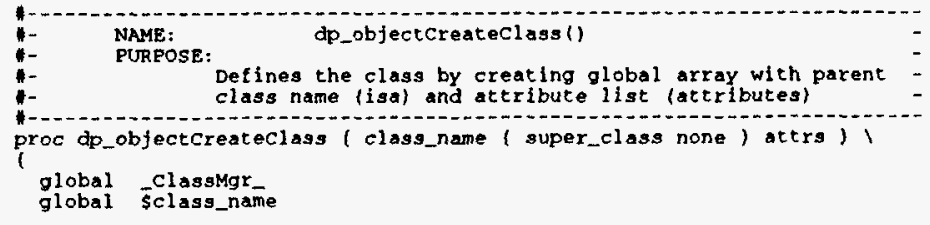

if (\$_Classmgr_(debug) ) ।

puts stdout ।

"idp_abjectcreateclassi) called with \$class_name, 5super_class, sattrs

set result " "

if ( info exists_Classmgr_(classes, \$class_name) ) )

puts stderr

I Error: class with name \$class name has al ready been declared.

elself $\{1$

"\$super_class" ! : "none" \&\&

(class) I ।

1

puts stderr '
"Error: superclass \$super_class has not been declared"

lse
- Set_classmgr_(classes, Sclass_name) Sclass_name

set \$(class_name) (isa) \$super_class
set \$(class_name) (attributes) Sattrs

- - - Build the Class Command For object Declarations proc sclass_name (args) ।

foreach item isargs \( dp_objectcreateobject \$class_name isitem U)

set result \$class_name

return sresult

ap_objectcreateclass

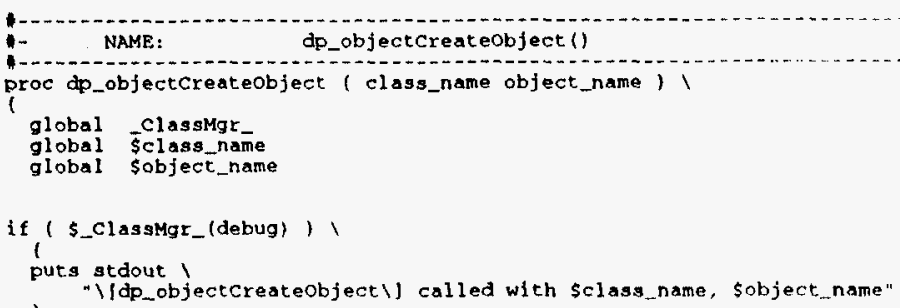

global ClassMgr
global Slass_name
global Sobject_name

if ( \$_classmgr_ (debug) )

puts "stdout '

set result " "

if ( , linfo exists _Classmgr_, (classes, Sclass_name)) ,

puts stderr "Error: class sclass_name has not been defined.

else 1

- set s(object_name)(isa) Sclass_name

- -- Build Class Hierarehy List

set class__list Sclass_name

for (set parent [set S(class_name) (isa)]; global sparent ) ।

( "Sparent" ! " "none" ) I )(isa)]; global sparent)
(set parent (set \$(parent)

lappend class_1ist sparent

- -- set object Attributes

(set index (expr [1length \$class_list] 1) )

( sindex $>=0$ )

set parent $[1$ index sclass 1 ist sindex 


\section{foreach item [set s(parent) (attributes)]}

if ( (set len lllength sitem) $>1)$ (set s(object_name)(lindex sitem ol) (IIndex sitem 1))

elseif ( slen $=1$ )

,

- proe sobject_name ( op args ) ।

dp_objectRunMethod \$object_name 1\$op 1\$args

set result sobject_name

return \$result

idpobjectcreateobject

NAME proc dp_objectexists ( object_name ) ।

if ( "[info proc sobject_name $] "==\cdots)($ return 0$)\}$

dp_objectExists

NAME:

proc dp_objectrree ( object_name)

global sobject_name

rename Sobject_name " "

)

dp_objectFree

NAME: de_objectslotFree 0

proc dp_abjectslot reee ( object_name slot)

global sobject_name

catch ( unset $\$$ (object_name) (\$slot) ),

dp_objectslotfree

NAME:
proc dp_objectslot ( object_name slot)

ject_name slot !

global sobject_name

return [set $\$$ (object_name) ( $\$$ slot)]

dp_objectslot name: dp_objectslotset ()
proc dp_objectslotset (object_name slot

'global sobject_name

return [set $\$$ (object_name) (sslot) svalue]

- dp_objectslotset

proc dp_objectslotappend ( object_name slot value ) ।
global sobject_name

global sobject_name

return [1append s(object_name)(sslot) svalue]:

- dp_objectslotappend

proc dp_objectsiots ( object_name) i

global sobject_name

return [array names sobject_name]

dp_objectslots

proc dp_objectConfigure (object_name args )
global sobject_name

set result " "
set arge [11ength sargs]

if $($ sarge $<1)$ )

foreach attr (array names sobject_namel I

lappend resule [1ist -\$attr () [set \$(object_name)(\$attr) )]

) ?

elseif ( sargc $==1)$ ।

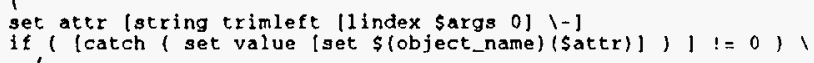

puts stderr "Error: slot sattr not defined for object sobject_name"

else

set result [1ist -\$attr o svalue]

else

incr arge - 1

for i set aindex 0 ) । 


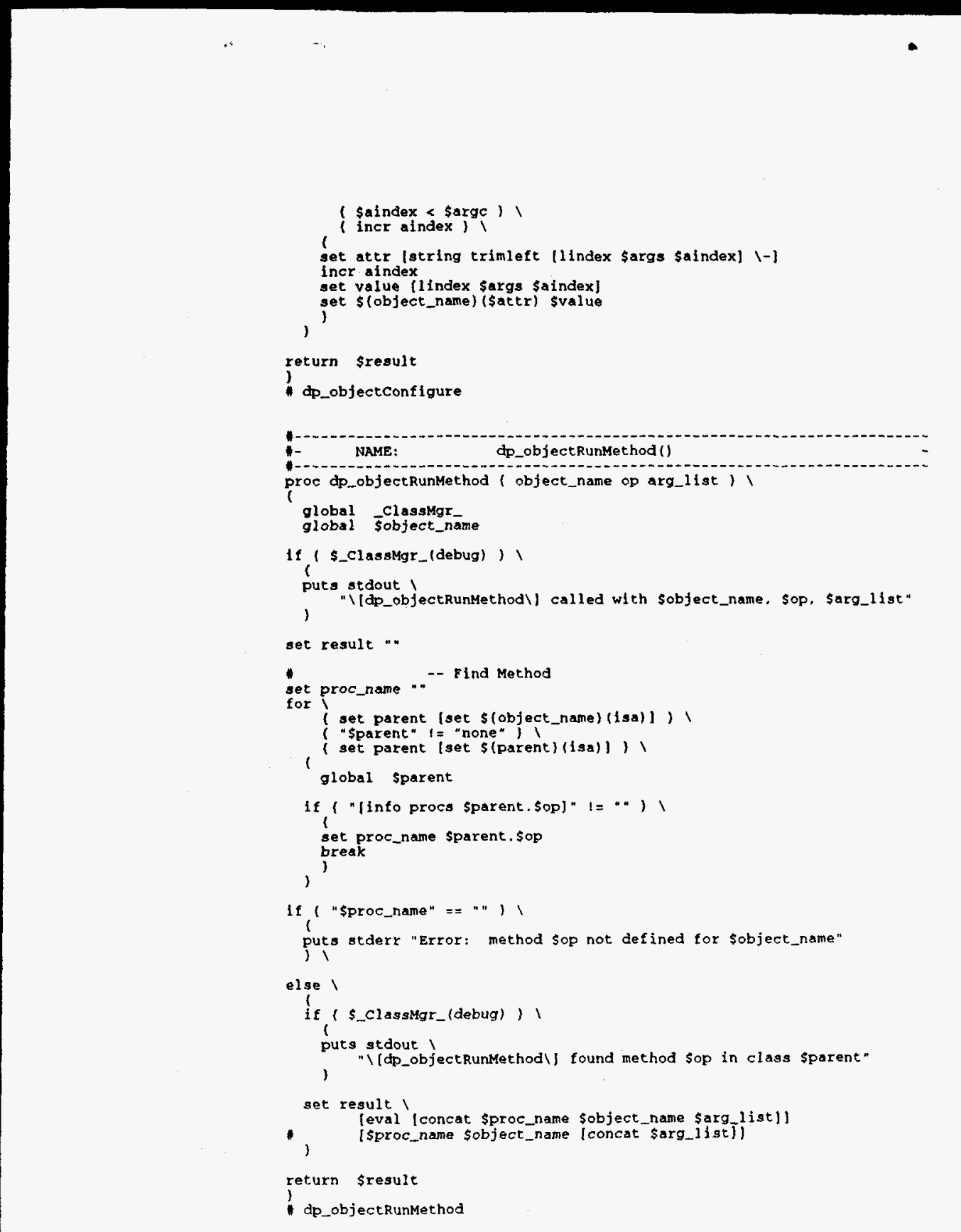




\section{INTERNAL DISTRIBUTION}

1. R. K Browning

2. C. W. Glover

3. R. Hume

4. W. R. Lee

5. D. M. Lopez

6. R. C. Mann

7. J. P. McNeely

8. E. M. Oblow

9. S. Petrov
10. J. A. Rome

11. M. B. Shah

12. R. A. Tannert, Jr.

13. E. C. Uberbacher

14. EPMD Reports Office

15-16. Laboratory Records, ORNL-RC

17. Document Reference Section

18. Central Research Library

19. ORNL Patent Office

\section{EXTERNAL DISTRIBUTION}

20-21. Office of Scientific and Technical Information, P.O. Box 62, Oak Ridge, TN 37831.

20. Office of Assistant Manager for Energy Research and Development, Oak Ridge Operations, U.S. Department of Energy, P.O. Box 2008, Oak Ridge, TN 37831

21. Jim Decker, Director, Office of Energy Research, Dept. of Energy, Washington, DC 20585

22. Prof. Donald J. Dudziak, Dept. of Nuclear Engineering, 110B Burlington Engineering Labs, North Carolina State University, Raleigh, NC 27695-7909.

23. Prof. Dr. James E. Leiss, Rt. 2, Box 142C, Broadway, VA 22815 